Draft VERSiOn SEPTEMBER 28, 2016

Preprint typeset using LATEX style AASTeX6 v. 1.0

\title{
OBSERVATIONAL CONSTRAINTS ON FIRST-STAR NUCLEOSYNTHESIS. I. EVIDENCE FOR MULTIPLE PROGENITORS OF CEMP-NO STARS
}

\author{
Jinmi Yoon ${ }^{1,2}$, Timothy C. Beers ${ }^{1,2}$, Vinicius M. PlacCO ${ }^{1,2}$, Kaitlin C. Rasmussen ${ }^{1,2}$ \\ Daniela Carollo ${ }^{1,2}$, Siyu He ${ }^{3}$, Terese T. Hansen ${ }^{4}$, Ian U. Roederer ${ }^{2,5}$, and Jeff Zeanah ${ }^{6}$

\footnotetext{
${ }^{1}$ Department of Physics, University of Notre Dame, Notre Dame, IN 46556, USA; jinmi.yoon@nd.edu

${ }^{2}$ Joint Institute for Nuclear Astrophysics - Center for the Evolution of the Elements (JINA-CEE), USA

${ }^{3}$ Department of Physics, Xi'an Jiaotong University, Shaanxi, 710049, People's Republic of China

${ }^{4}$ Observatories of the Carnegie Institution of Washington, 813 Santa Barbara St., Pasadena, CA 91101, USA

${ }^{5}$ Department of Astronomy, University of Michigan, 1085 S. University Avenue, Ann Arbor, MI 48109, USA

${ }^{6}$ Z Solutions, Inc., 9430 Huntcliff Trace, Atlanta, GA 30350, USA
}

\begin{abstract}
We investigate anew the distribution of absolute carbon abundance, $A(\mathrm{C})=\log \epsilon(\mathrm{C})$, for carbonenhanced metal-poor (CEMP) stars in the halo of the Milky Way, based on high-resolution spectroscopic data for a total sample of 305 CEMP stars. The sample includes 147 CEMP-s (and CEMPr/s) stars, 127 CEMP-no stars, and 31 CEMP stars that are unclassified, based on the currently employed $[\mathrm{Ba} / \mathrm{Fe}]$ criterion. We confirm previous claims that the distribution of $A(\mathrm{C})$ for CEMP stars is (at least) bimodal, with newly determined peaks centered on $A(\mathrm{C})=7.96$ (the high- $\mathrm{C}$ region) and $A(\mathrm{C})=6.28$ (the low-C region). A very high fraction of CEMP-s (and CEMP-r/s) stars belong to the high-C region, while the great majority of $\mathrm{CEMP}-$ no stars reside in the low-C region. However, there exists complexity in the morphology of the $A(\mathrm{C})-[\mathrm{Fe} / \mathrm{H}]$ space for the CEMP-no stars, a first indication that more than one class of first-generation stellar progenitors may be required to account for their observed abundances. The two groups of CEMP-no stars we identify exhibit clearly different locations in the $A(\mathrm{Na})-A(\mathrm{C})$ and $A(\mathrm{Mg})-A(\mathrm{C})$ spaces, also suggesting multiple progenitors. The clear distinction in $A(\mathrm{C}$ ) between the CEMP-s (and CEMP-r/s) stars and the CEMP-no stars appears to be as successful, and likely more astrophysically fundamental, for the separation of these sub-classes as the previously recommended criterion based on $[\mathrm{Ba} / \mathrm{Fe}]$ (and $[\mathrm{Ba} / \mathrm{Eu}]$ ) abundance ratios. This result opens the window for its application to present and future large-scale low- and medium-resolution spectroscopic surveys.
\end{abstract}

Keywords: stars: abundances — stars: chemically peculiar — stars: Population II — stars: AGB — stars: massive — stars: evolution - Galaxy: halo

\section{INTRODUCTION}

At low iron abundances relative to the Sun, a substantial fraction of the stars in the halo of the Milky Way have been found to be greatly enhanced in carbon, the so-called carbon-enhanced metal-poor (CEMP) stars. Beers \& Christlieb (2005) originally divided such stars into several sub-classes, depending on the nature of their neutron-capture element abundance ratios - CEMP-s, CEMP- $r$, CEMP-r $/ s$, and CEMP-no ${ }^{1}$. As discussed by these authors, and many since, the observed differences

\footnotetext{
${ }^{1}$ CEMP- $s:[\mathrm{C} / \mathrm{Fe}]>+1.0,[\mathrm{Ba} / \mathrm{Fe}]>+1.0$, and $[\mathrm{Ba} / \mathrm{Eu}]>$ $+0.5$

CEMP- $r:[\mathrm{C} / \mathrm{Fe}]>+1.0$ and $[\mathrm{Eu} / \mathrm{Fe}]>+1.0$

CEMP-r $/ s:[\mathrm{C} / \mathrm{Fe}]>+1.0$ and $0.0<[\mathrm{Ba} / \mathrm{Eu}]<+0.5$

CEMP-no : $[\mathrm{C} / \mathrm{Fe}]>+1.0$ and $[\mathrm{Ba} / \mathrm{Fe}]<0.0$
}

in the chemical signatures of the sub-classes of CEMP stars are thought to be tied to differences in the astrophysical sites responsible for the nucleosynthesis products they now incorporate in their atmospheres, including elements produced by the very first generations of stars.

\subsection{The Origin of CEMP Stars}

In this paper we focus on the two most populous subclasses of the carbon-enhanced metal-poor stars, the CEMP-s and CEMP-no stars. Based on both extensive observational follow-up and theoretical modeling, the elemental abundance pattern associated with the CEMP- $s$ stars (carbon enhancement accompanied by strong overabundances of neutron-capture elements produced by the main $s$-process) is thought to arise from an extrin- 
sic process - mass transfer to the presently observed star from an evolved binary companion. This companion, the site where the enhancement of carbon and the $s$-process elements originally took place, is expected to have been a low- to intermediate-mass $\left(\sim 1\right.$ to $\left.\sim 4 M_{\odot}\right)$ asymptotic giant-branch (AGB) star, which has now evolved to become a faint white dwarf (e.g., Suda et al. 2004; Herwig 2005; Lucatello et al. 2005b; Bisterzo et al. 2011; Starkenburg et al. 2014; Hansen et al. 2015a). The masstransfer process itself has proven challenging to model, despite extensive efforts in recent years (see, e.g., Abate et al. 2013, 2015a,c, and references therein).

Accreted material can potentially be mixed into the atmosphere of the presently observed companion by several processes (e.g., thermohaline mixing, levitation, etc., see Stancliffe et al. 2007; Stancliffe \& Glebbeek 2008; Stancliffe 2010; Matrozis \& Stancliffe 2016). Additional processing can also occur towards the tip of the giant branch (Placco et al. 2013; Karakas \& Lattanzio 2014; Placco et al. 2014b), all of which may complicate interpretation of the observed elemental-abundance patterns.

Binary mass transfer is thought to play a role in the origin of the CEMP-r/s stars as well (e.g., Jonsell et al. 2006; Lugaro et al. 2009; Bisterzo et al. 2011; Herwig et al. 2011), but the origin of the $r$-process-like component of their abundance pattern remains unclear (Abate et al. 2016). It remains possible that yet another nucleosynthetic process, the so-called $i$-process, may need to be invoked to account for their observed abundance patterns (Dardelet et al. 2015; Hampel et al. 2016). For the purpose of the present analysis, we group the CEMP- $r / s$ stars along with the CEMP-s stars.

In contrast to the CEMP- $s$ and CEMP-r/s stars, a number of lines of observational evidence (including long-term radial-velocity monitoring; see Hansen et al. 2016b) indicate that the distinctive abundance patterns of CEMP-no stars (carbon enhancement with a lack of neutron-capture element over-abundances) arose from an intrinsic process $^{2}$. The inference is that the presently observed CEMP-no stars are indeed bona-fide secondgeneration stars, born in natal clouds polluted by massive first-generation stars. A number of astrophysical sites for the progenitors of the CEMP-no stars have been suggested. The so-called "faint supernovae" or "mixingand-fallback" models (Umeda \& Nomoto 2003, 2005; Nomoto et al. 2013; Tominaga et al. 2014) hold that the gas from which CEMP-no stars formed was enriched by

\footnotetext{
${ }^{2}$ We intend this term to indicate that the observed elemental abundances on the surface of the star were present in the gas from which the star first formed, and not (as it is also used) patterns arising from internal processing in the star of material that is later transported to the stellar surface.
}

a supernova without sufficient explosion energy to release its full complement of synthesized heavy elements (which fall back to the nascent neutron star or black hole at its center), and only the lighter elements (including $\mathrm{C}, \mathrm{N}, \mathrm{O}$, and other light elements such as $\mathrm{Na}, \mathrm{Mg}, \mathrm{Al}$, and $\mathrm{Si}$ ) are expelled. Another possibility, the so-called "spinstar" model (e.g., Meynet et al. 2006, 2010; Chiappini 2013) proposes that the gas from which CEMP-no stars formed was enhanced in carbon (as well as $\mathrm{N}$ and O) by the strong stellar winds expected to arise from rapidly-rotating massive stars of ultra low metallicity. In addition, Heger \& Woosley (2010) have considered possible progenitors of the CEMP-no stars including the effects of rotation and mixing and fallback. More recent modeling has suggested that spinstars may also be capable of producing other light elements and some amount of first-peak neutron-capture elements (such as $\mathrm{Sr}$ ) and second-peak $s$-process elements (such as Ba), and possibly even third-peak elements such as $\mathrm{Pb}$, depending on the degree of the internal mixing induced by the rapid rotation (Maeder \& Meynet 2015; Frischknecht et al. 2016).

Finally, we note that Cooke et al. (2011, 2012) have reported on recently discovered high-redshift carbon-enhanced damped Lyman- $\alpha$ systems that exhibit elemental-abundance patterns which resemble those expected from massive, carbon-producing first stars. These authors speculated that the progenitors that produced these patterns are the same as those responsible for those associated with CEMP-no stars in the Galaxy.

\subsection{The High and Low Carbon Bands for CEMP Stars}

Spite et al. (2013) used literature abundance data for $\sim 50$ CEMP main-sequence turnoff and dwarf stars, including both CEMP-s and CEMP-no stars, and plotted the absolute carbon abundance, $A(\mathrm{C})=\log \epsilon(\mathrm{C})^{3}$, as a function of metallicity, $[\mathrm{Fe} / \mathrm{H}]$, for their sample. The stars in their sample were specifically chosen to be in evolutionary stages where alteration of their surface elemental abundances, due to significant internal mixing, were not expected to have occurred. We point out, however, that certain processes, such as thermohaline mixing, occur almost immediately after mass-transfer events (in CEMP-s stars; R. Stancliffe, priv. comm.), so some mixing (dilution) may have occured even in supposedly unevolved stars.

Based on this sample, they claimed the existence of a clear bimodality among the CEMP stars - the stars in their sample with $[\mathrm{Fe} / \mathrm{H}]>-3.0$, which are dominated by CEMP-s stars, populate a high-C "plateau" at $A(\mathrm{C})$

\footnotetext{
${ }^{3} A(\mathrm{X})=\log \epsilon(\mathrm{X})=\log \left(N_{\mathrm{X}} / N_{\mathrm{H}}\right)+12$, where $\mathrm{X}$ represents a given element.
} 
$\sim 8.25$, close to the Solar value of $A(\mathrm{C})$. In contrast, the stars with $[\mathrm{Fe} / \mathrm{H}]<-3.4$, which are exclusively CEMPno stars, reside in a lower region (and possible plateau) at $A(\mathrm{C}) \sim 6.5$. They interpreted this behavior as the result of the different carbon-production mechanisms for these sub-classes of stars - mass-transfer from binary AGB companions in the case of CEMP- $s$ stars and enrichment of the natal clouds of the CEMP-no stars by massive-star nucleosynthesis.

Bonifacio et al. (2015) confirmed and extended the claim by Spite et al. with a larger sample $(\sim 70)$ of unevolved main-sequence turnoff and dwarf stars, along with a few lower red giant-branch (RGB) CEMP stars with $[\mathrm{Fe} / \mathrm{H}]>-3.5$. These authors found a clear separation of the $A(\mathrm{C})$ distribution, but commented that the individual distributions of carbon abundance were quite wide, on the order of one dex. They advocated for a similar explanation of this separation as in Spite et al., based on different carbon-production mechanisms for the CEMP-s and CEMP-no stars.

The work of Hansen et al. (2015a) provided new data for additional CEMP stars, and considered them along with literature data (compilation from Yong et al. 2013), confirming once again the existence of the carbon bands, based on a total of 64 stars. However, they identified three CEMP-no stars located on the high-C band, as well as the apparent existence of a smooth transition of $A(\mathrm{C})$ between the two bands, which as they noted presents a challenge to the interpretation of the bimodality in $A(\mathrm{C})$ as exclusively due to extrinsic (AGB mass-transfer) and intrinsic (C-enriched ISM) processes. These authors emphasized the crucial role that knowledge of the binary status for stars associated with the two carbon bands may play for determination of the nature of their progenitors, and recently published the results of long-term radial-velocity monitoring for samples of CEMP-no (Hansen et al. 2016b) and CEMP-s (Hansen et al. 2016c) stars.

In order to further explore these questions, we have compiled an extensive set of 305 CEMP stars with available high-resolution spectroscopic data from the literature, including more recent data than was available to the studies conducted in the past few years.

This paper is arranged as follows. Section 2 describes details of the literature data compilation, and the corrections we have applied in order to place the data on a suitable common scale. The results of our analysis, presented in Section 3, clearly support the existence of (at least) a bimodality in the distribution of $A(\mathrm{C})$ for CEMP stars, but we note that the $A(\mathrm{C})$ distribution exhibits more complex behavior that is not captured by its description as carbon plateaus or bands. Instead, we suggest that the CEMP stars can be more usefully described as falling into three groups, one for the CEMP-s (and
CEMP-r $/ s$ ) stars and two for the CEMP-no stars, based on their location in the $A(\mathrm{C})-[\mathrm{Fe} / \mathrm{H}]$ space. We discuss these divisions in more detail in Section 4, and demonstrate the existence of a correlated behavior between the absolute abundances of the light elements $\mathrm{Na}$ and $\mathrm{Mg}$, $A(\mathrm{Na})$ and $A(\mathrm{Mg})$, with $A(\mathrm{C})$. Collectively, this may provide the first evidence for the existence of at least two distinct progenitor populations that are responsible for the abundance signatures among CEMP-no stars. In this section we also consider information that can be gleaned from the subset of CEMP stars with known binary status, concluding that the carbon enhancement of the great majority of CEMP-no stars is an intrinsic process, while most CEMP- $s$ (and CEMP- $r / s$ ) stars are extrinsically enriched, as previously suggested. We also identify several interesting subsets of stars that exhibit abundance anomalies relative to the majority of other CEMP stars in our sample. Finally, we argue that classification based on $A(\mathrm{C})$ is likely to be a more astrophysically fundamental (and equally successful) method to distinguish the CEMP-no stars from the CEMP-s and CEMP- $r / s$ stars than the previously employed approach based on $[\mathrm{Ba} / \mathrm{Fe}]$ (and $[\mathrm{Ba} / \mathrm{Eu}]$ ) ratios, with the considerable advantage that it can be obtained from lowto medium-resolution, rather than high-resolution, spectroscopy. Our conclusions are briefly summarized in Section 5 .

\section{COMPILATION OF LITERATURE DATA}

We have endeavored to compile as complete a list as possible of CEMP-s (and CEMP-r/s) and CEMP-no stars having $[\mathrm{Fe} / \mathrm{H}]<-1.0$ and $[\mathrm{C} / \mathrm{Fe}] \geq+0.7$ with available high-resolution spectroscopic abundance information. We have only considered stars with claimed detections or lower limits for carbon, along with several critical elemental abundance ratios, such as $[\mathrm{Ba} / \mathrm{Fe}]$ and $[\mathrm{Eu} / \mathrm{Fe}]$. The great majority of our sample comes from the literature compilation of Placco et al. (2014b). To this, we have added more recent literature data from a number of authors (e.g., Roederer et al. 2014b; Hansen et al. 2015a; Jacobson et al. 2015), as well as a number of more metal-rich CEMP stars (sometimes referred to as "CH stars") with $-2.0<[\mathrm{Fe} / \mathrm{H}] \leq-1.0,[\mathrm{C} / \mathrm{Fe}]$ $\geq+0.7$, and $[\mathrm{Ba} / \mathrm{Fe}] \geq+1.0$. Our sample of these more metal-rich CEMP stars is certain to be incomplete. For stars with multiple reported observations, we have given preference to those stars with spectra having higher resolving power and/or higher $\mathrm{S} / \mathrm{N}$; for those with similar quality spectra, we kept the most recent data.

Because we compiled the literature data from studies conducted with a variety of instruments, and analyzed with different atmospheric models and methods of estimation, there are unavoidable inconsistencies in the adopted abundance estimates. There are also dif- 
ferences between authors in the assignment of the subclasses for CEMP stars, in particular for the stars with $0.0<[\mathrm{Ba} / \mathrm{Fe}]<+1.0$. Below we describe our attempt to resolve at least some of these difficulties.

High-resolution spectroscopic analyses of metal-poor stars conventionally estimate effective temperatures using broadband photometric colors (e.g., $B-V$ or $J-K$ ), however, some studies (e.g., Roederer et al. 2014b) have chosen to estimate $\mathrm{T}_{\text {eff }}$ from the spectral lines themselves. It has long been recognized that there exist systematic offsets between effective temperatures estimated by these two methods (e.g., Frebel et al. 2013). When available, we collected the reported $A(\mathrm{C})=\log \epsilon(\mathrm{C})$ from each study. For some studies, e.g., Cohen et al. (2013), as well as the compilation of Placco et al. (2014b), only $[\mathrm{C} / \mathrm{Fe}]$ estimates were reported. In these cases, estimates of absolute carbon abundances, $A(\mathrm{C})$, were derived using the relation $A(\mathrm{C})=[\mathrm{C} / \mathrm{Fe}]+[\mathrm{Fe} / \mathrm{H}]$ $+A(\mathrm{C})_{\odot}$. The systematic errors in the absolute abundance of $A(\mathrm{C})$ introduced by this, for $\mathrm{T}_{\text {eff }}$ differences up to $200-250 \mathrm{~K}$, are within the typical observational errors for $A(\mathrm{C}) \sim 0.20-0.25 \mathrm{dex}$; we make no explicit correction for their presence. However, these do not have a major effect on derived elemental abundance ratios, $[\mathrm{X} / \mathrm{Fe}]$, which are similarly affected by temperature variations. $^{4}$

The overall stellar metallicity, $[\mathrm{Fe} / \mathrm{H}]$, is often represented by Fe II lines, when feasible, because they represent the dominant ionization state for the temperature range of F-G-K stars. However, for stars with metallicities with $[\mathrm{Fe} / \mathrm{H}]<-3.0$, the $\mathrm{Fe}$ II lines are rarely sufficiently numerous (or even detectable), hence many studies have been forced to use neutral iron lines. Nevertheless, the Roederer et al. (2014b) study was able to derive metallicities based on the Fe II lines, owing to the relatively bright stars in their sample. In addition, when they calculated the individual element abundance ratios they used the same ionization state of iron as for the element under consideration - for instance, [Ba II/Fe II] for the $\mathrm{Ba} / \mathrm{Fe}$ abundance ratio. In order to be consistent with the majority of studies in our compilation, we have recalculated $[\mathrm{Fe} / \mathrm{H}]$ and the relative elemental abundance ratios $([\mathrm{X} / \mathrm{Fe}])$ based on $\mathrm{Fe} \mathrm{I}$ for this study. We have also only considered results based on 1-D LTE assumptions. All metallicities and abundances were rescaled to the Asplund et al. (2009) Solar photospheric abundances.

Evolved stars are known to have some degree of de-

\footnotetext{
4 The typical systematic error of $A(\mathrm{Fe})$, due to a temperature variation of $\sim 200-250 \mathrm{~K}$ is, in general, comparable to those of $A(\mathrm{C})$. The net effects on the abundance ratios are not significant when compared to the typical total errors, $\sim 0.2-0.25$ dex, in $[\mathrm{C} / \mathrm{Fe}]$.
}

pletion in their surface carbon abundances due to $\mathrm{CN}$ processing that takes place both because of first dredgeup and additional mixing on the upper RGB (Gratton et al. 2000). Therefore, for such stars, we have estimated the "original" carbon abundances based on each star's evolutionary state using an online carbon-correction calculator $^{5}$. For the stars added to our compilation since Placco et al. (2014b), and provided without classifications by the original authors, we have assigned CEMP sub-classes based on the definitions of Beers \& Christlieb (2005), but using $[\mathrm{C} / \mathrm{Fe}] \geq+0.7$ (Aoki et al. 2007) as the criterion for carbon enhancement. We note that some studies (e.g., Masseron et al. 2010; Spite et al. 2013; Bonifacio et al. 2015; Hansen et al. 2016c) classified CEMP sub-classes somewhat differently for the CEMP stars with $0.0<[\mathrm{Ba} / \mathrm{Fe}]<+1.0$; we adopted their claimed classification.

Our full sample consists of 305 CEMP stars: 147 CEMP-s/rs stars (hereafter, we employ this notation to include both the CEMP-s and CEMP-r/s stars), 127 CEMP-no stars, and 31 CEMP stars that are not subclassified by the conventional criteria. Table 1 lists the stellar parameter estimates for our program sample in columns (2)-(4). Column (5) lists the reported carbon abundance ratio, $[\mathrm{C} / \mathrm{Fe}]$; column (6) is the corrected carbon abundance ratio, $[\mathrm{C} / \mathrm{Fe}]_{c}$; and column $(7)$ is the absolute carbon abundance, $A(\mathrm{C})$, based on the corrected carbon abundance ratio. Columns (8) and (9) are the reported $[\mathrm{Ba} / \mathrm{Fe}]$ and $[\mathrm{Eu} / \mathrm{Fe}]$ ratios, respectively, corrected in some cases as described above. Below we discuss an alternative classification scheme for CEMP stars, based on $A(\mathrm{C})$, rather than one that requires use of the $[\mathrm{Ba} / \mathrm{Fe}]$ ratio. Column (10) of Table 1 , labeled as "Class (Ba| $A(\mathrm{C}))$ ", lists the sub-classification of a given star based first on the $[\mathrm{Ba} / \mathrm{Fe}]$ criterion, followed by its classification based on the $A(\mathrm{C})$ criterion described below. For instance, the notation (no $\mid s$ ) means that the star was sub-classified as a CEMP-no star based on the $[\mathrm{Ba} / \mathrm{Fe}]$ criterion, but as a CEMP-s/rs star based on the $A(\mathrm{C})$ criterion. We note that precise sub-classification for these stars (as either CEMP-s or CEMP-r/s) requires a $[\mathrm{Eu} / \mathrm{Fe}]$ detection, which is not presently available for all of the stars in our sample. The binary status of a given star, if known, is listed in column (11). We also indicate cases of stars with disparate $A(\mathrm{C})$ abundances and $[\mathrm{Ba} / \mathrm{Fe}]$ abundance ratios (for instance, high $A(\mathrm{C})$ with low $[\mathrm{Ba} / \mathrm{Fe}])$, including stars with anomalous $A(\mathrm{C})$ or $[\mathrm{Ba} / \mathrm{Fe}]$ given their known binary status. We marked these objects with a $\sqrt{ }$ in column (12) as "In-

\footnotetext{
5 http://www.nd.edu/ vplacco/carbon-cor.html
} 


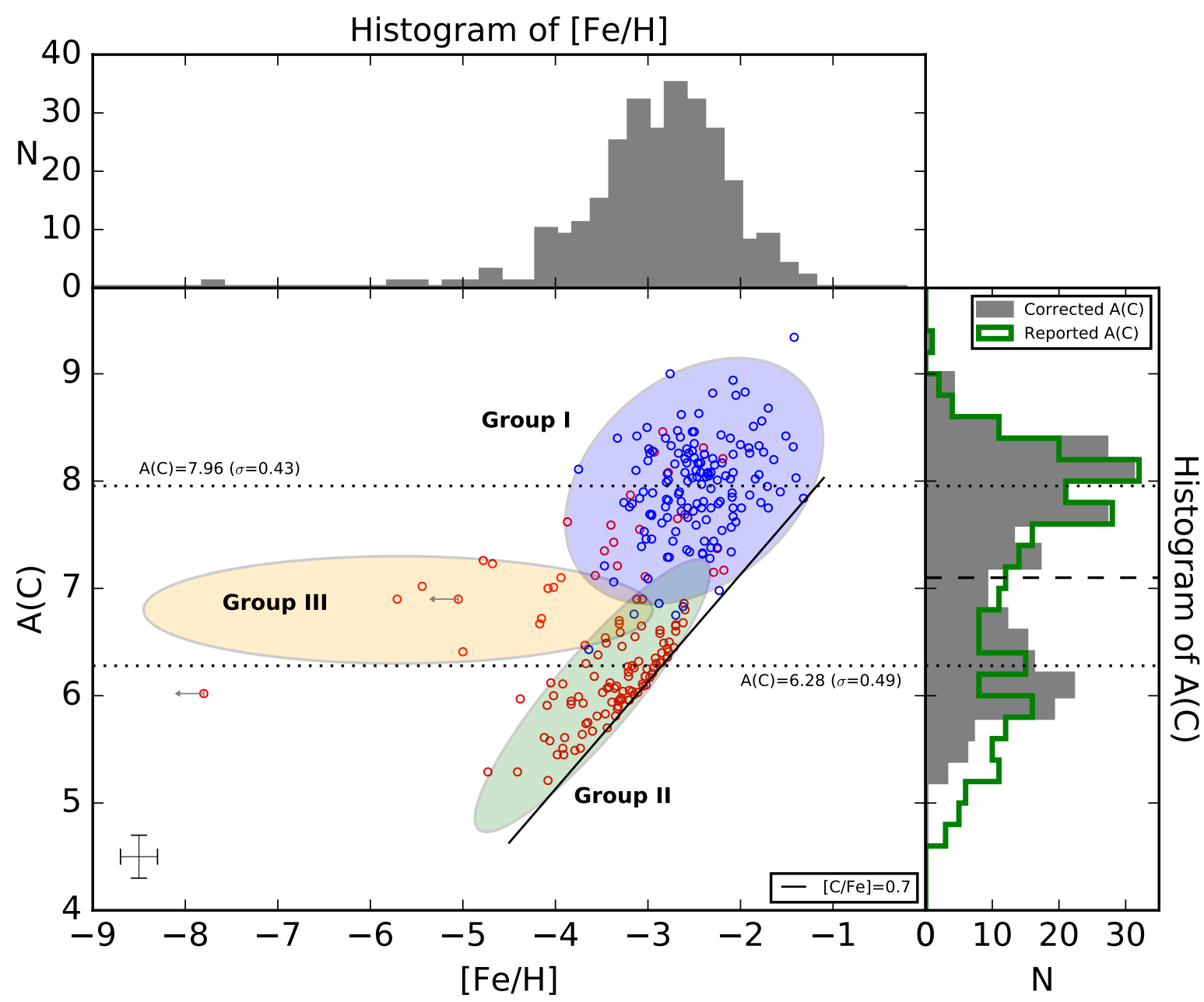

Figure 1. Scatter diagram of the corrected $A(\mathrm{C})$ vs. $[\mathrm{Fe} / \mathrm{H}]$ for our compilation of CEMP stars. The blue and red open circles represent the 147 CEMP-s/rs stars and 127 CEMP-no stars, respectively (the 31 unclassified CEMP stars are not shown). The black dotted lines indicate the estimated locations of the carbon peaks, based on a two-component Gaussian fit to the corrected $A(\mathrm{C})$ distribution. The majority of the CEMP-s/rs stars reside in a region surrounding the high-C peak at $A(\mathrm{C}) \sim 8.0$, while the majority of CEMP-no stars scatter around the low-C peak at $A(\mathrm{C}) \sim 6.3$. The black solid line provides a reference at $[\mathrm{C} / \mathrm{Fe}]=+0.7$. The gray shaded histogram in the top margin shows the metallicity distribution of the full sample. The gray shaded histogram in the right margin is the corrected $A(\mathrm{C})$ distribution; the green unfilled histogram is the "as reported" $A(\mathrm{C})$ distribution. Note that the 31 unclassified CEMP stars are not included in these fits. The black dashed line in the marginal histogram of $A(\mathrm{C})$ represents the midpoint of the two $A(\mathrm{C})$ peaks, used for separation of CEMP-s/rs stars from CEMP-no stars, as described in the text. A typical error bar for the sample stars we consider is shown at the bottom left.

teresting outliers" ${ }^{6}$. Column (13) of the table provides the reference to the original study from which our stellar parameter and abundance information was drawn.

For convenience of the subsequent analysis, we have listed our program stars in Table 1 in the groupings described in Section 3 below (Group I, Group II, and Group III, along with a group without subclassifications based on $[\mathrm{Ba} / \mathrm{Fe}])$.

${ }^{6}$ We group these stars along with other outliers having similar behavior in Table 3, as described below.

\section{RESULTS}

Figure 1 shows the corrected $A(\mathrm{C})$ distribution, as a function of $[\mathrm{Fe} / \mathrm{H}]$, for our compiled sample of subclassified CEMP (147 CEMP-s/rs and 127 CEMP-no) stars. The remaining 31 unclassified CEMP stars are not included in Figure 1 and subsequent figures, due to their lack of available $[\mathrm{Ba} / \mathrm{Fe}]$ measurements. The blue and red open circles represent the CEMP-s/rs and CEMP-no stars, respectively. Note from the marginal histogram shown on the right side of the figure that a significant number of the stars among the CEMP-no sub-sample (those with the lowest $A(\mathrm{C})$, which generally correspond to the more evolved, higher luminosity 

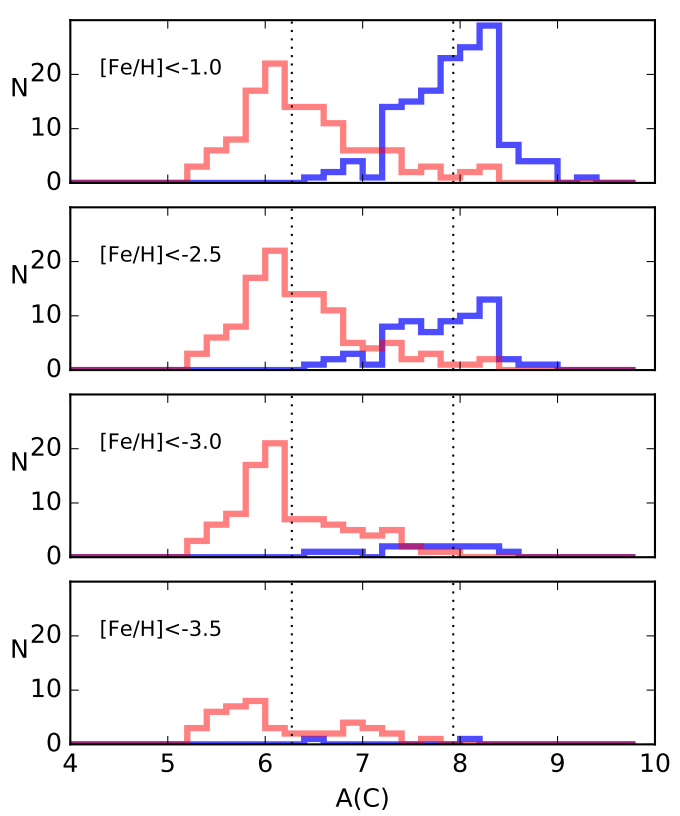

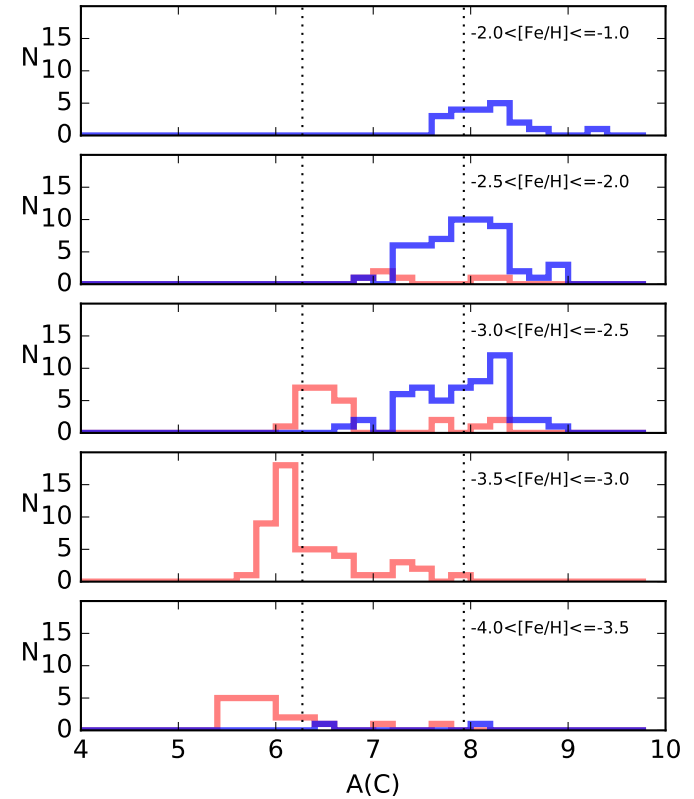

Figure 2. Left panel: Cumulative histograms of $A(\mathrm{C})$ for the the CEMP-s/rs stars (blue) and CEMP-no stars (red), in cuts of decreasing metallicity. Right panel: Differential histograms of $A(\mathrm{C})$ for both sub-classes in intervals of declining metallicities. The dotted black lines represent the estimated location of the high-C and low-C peaks based on a two-component Gaussian fit (see text).

giants that undergo $\mathrm{CN}$ processing) have $A(\mathrm{C})$ values that have been revised upward relative to their "as reported" values, whereas the CEMP-s/rs stars have only a small number of corrections applied (see Placco et al. 2014b). The bimodality of the $A(\mathrm{C})$ distribution is much clearer post correction, underscoring the importance of carrying out this step.

Although the bimodality of $A(C)$ is clear in Figure 1, the peaks of the distribution are about 0.3 dex lower than the determinations by Spite et al. (2013), who claimed $A(\mathrm{C}) \sim 8.25$ and $A(\mathrm{C}) \sim 6.5$ for the CEMP$s$ and CEMP-no stars, respectively ${ }^{7}$. We obtained estimates of the peaks in our distribution by fitting a two-component Gaussian distribution (Pedregosa et al. 2011) to the corrected $A(\mathrm{C})$ values, obtaining peak values of $A(\mathrm{C})=7.96$ (with a dispersion of $0.43 \mathrm{dex}$ ) for the high-C region, and $A(\mathrm{C})=6.28$ (with a dispersion of $0.49 \mathrm{dex}$ ) for the low-C region, respectively. These peaks are indicated by the black dotted lines in the figure; the black dashed line shown in the marginal histogram of $A(\mathrm{C})$ is located at the midpoint between these peaks, at $A(\mathrm{C})=7.1$. The previous claim that most CEMP-

\footnotetext{
7 It should be recalled that Spite et al. (2013) considered only the "unmixed" CEMP stars in their discussion, whereas, in our attempt to build a larger total sample, we have included both unevolved and evolved (giant) stars. When only unevolved stars $(\sim 120$ stars with logg $>2.5)$ in our sample are taken into account for the peak estimates, the low-C peak is located at $\mathrm{A}(\mathrm{C}) \sim 6.6$, similar to the location reported by Spite et al.
}

$s / r s$ stars are associated with the high-C region, while the majority of CEMP-no stars are associated with the low-C region, is clearly supported.

There are remarkable differences in the morphology of the $A(\mathrm{C})$ vs. $[\mathrm{Fe} / \mathrm{H}]$ distributions between the CEMP$s / r s$ stars and the CEMP-no stars, as seen from inspection of Figure 1. The distribution of $A(\mathrm{C})$ for the CEMP-s/rs stars, indicated with the blue shaded ellipse in Figure 1, exhibits a very weak dependence on $[\mathrm{Fe} / \mathrm{H}]-$ there exists a wide scatter of $A(\mathrm{C})$ values for these stars at any given $[\mathrm{Fe} / \mathrm{H}]$. We refer to this subset of stars in the $A(\mathrm{C})$ vs. $[\mathrm{Fe} / \mathrm{H}]$ diagram as "Group I" stars. In contrast, the CEMP-no stars exhibit two very different behaviors in the $A(\mathrm{C})$ vs. $[\mathrm{Fe} / \mathrm{H}]$ space. For convenience, we refer to these as "Group II" and "Group III" stars $^{8}$ and indicate them in the figure with the green and orange shaded ellipses, respectively. Note that all three groups are defined based on their morphology in the $A(\mathrm{C})$ vs. $[\mathrm{Fe} / \mathrm{H}]$ space alone; each group contains a small fraction of CEMP stars with sub-classifications

8 We note that application of objective clustering procedures yielded similar results. Three elliptical clusters were determined using the mclust routine (Fraley \& Raftery 2002; Fraley et al. 2012), implemented in R using the EM Mixed Model algorithm and the Bayesian Information Criterion (BIC). The algorithm was constrained to select three clusters. If left unconstrained, the algorithm would further subdivide the data points of Group II, however, the apparent sub-structure in this group is likely introduced by selection effects in the observations made to date, hence it was suppressed. 
that differ from the majority within the group - these are discussed in more detail below. The stars are listed by these different morphological groups in Table $1 .{ }^{9}$

The Group III stars are located in the lowest metallicity regime, $[\mathrm{Fe} / \mathrm{H}]<-3.5$, centered on $A(\mathrm{C}) \sim 6.8$, higher than the $A(\mathrm{C})$ values for many of the Group II stars. The $A(\mathrm{C})$ values for the Group III stars also exhibit no clear dependence on $[\mathrm{Fe} / \mathrm{H}]$. We note that the lowest $[\mathrm{Fe} / \mathrm{H}]$ star in our sample (SMSS J0313-6708, with $[\mathrm{Fe} / \mathrm{H}]<-7.8$ ) has a lower $A(\mathrm{C}$ ) (by about 0.3 dex) than the rest of the Group III stars. We assign this star to Group III, as its location is more similar to other Group III stars than to the Group II stars.

The Group II stars are clustered in the metallicity range $-5.0 \lesssim[\mathrm{Fe} / \mathrm{H}] \lesssim-2.5$, and, in contrast to the Group III stars, exhibit a clear dependence of $A(\mathrm{C})$ on $[\mathrm{Fe} / \mathrm{H}]$. As seen in the figure, there are also CEMP-no stars that fall into neither of these groups, but are scattered throughout the region primarily occupied by the CEMP-s/rs stars - these stars are placed into Group I.

There also exists a contrast in the metallicity distributions between the CEMP-s/rs and CEMP-no stars, as seen in Figure 1. The CEMP-s/rs stars in our sample cover a range of $\sim 2.5$ dex in metallicity, with the mean at $[\mathrm{Fe} / \mathrm{H}]=-2.47$ (recall that our sample is incomplete at the metal-rich end, so the mean value is likely somewhat higher in reality). By comparison, the CEMP-no stars are spread over $\sim 6$ dex in metallicity, with the mean at $[\mathrm{Fe} / \mathrm{H}]=-3.42$. This contrast is seen in Figure 2 as well. The left panels are cumulative histograms of $A(\mathrm{C})$ for each CEMP sub-class over different cuts of decreasing $[\mathrm{Fe} / \mathrm{H}]$. The right panels are differential histograms of $A(\mathrm{C})$ for the CEMP sub-classes in specific metallicity ranges. As claimed in Spite et al. (2013), most of the CEMP-s/rs stars reside in the high-C region, while the CEMP-no stars predominantly belong to the low-C region, as shown in the top left panel, although they are asymmetrically distributed, with a long tail toward higher $A(\mathrm{C})$ in all the metallicity cuts. As noted by previous work (e.g., Aoki et al. 2007; Placco et al. 2014b), the CEMP-no stars are dominant in the metallicity range $[\mathrm{Fe} / \mathrm{H}]<-3.0$, while most CEMP$s / r s$ stars are found above this metallicity, consistent with the behavior of both the cumulative and differential histograms shown in Figure 2. We note that there are two CEMP- $s$ stars in our sample with quite low metallicities compared to the rest of the CEMP-s/rs stars, CS 22960-053 with $[\mathrm{Fe} / \mathrm{H}]=-3.64$ (Roederer et al. 2014b) and HE 0002-1037 with $[\mathrm{Fe} / \mathrm{H}]=-3.75$ (Hansen et al. 2016c).

9 The stars in the overlapping regions are also listed as such in the table notes.

\section{DISCUSSION}

In this section we consider the astrophysical implications of the observed $A(\mathrm{C})$ vs. $[\mathrm{Fe} / \mathrm{H}]$ distributions for CEMP stars, and speculate on what more might be revealed from further detailed studies. We also summarize the known binary status of our sample of CEMP stars, and highlight several cases of "interesting outliers" stars whose binary status does not meet with expectation given their observed $A(\mathrm{C})$ and/or $[\mathrm{Ba} / \mathrm{Fe}]$, or with disparate values of $A(\mathrm{C})$ and $[\mathrm{Ba} / \mathrm{Fe}]$. Finally, we consider whether $A(\mathrm{C})$ can be used as an effective discriminator between the dominant populations of CEMP stars, as an alternative to the $[\mathrm{Ba} / \mathrm{Fe}]$-based scheme for CEMP sub-classification that is in conventional use.

\subsection{The Complex Behaviors in the $A(\mathrm{C})$ vs. $[\mathrm{Fe} / \mathrm{H}]$ Distribution}

The different behaviors shown in the $A(\mathrm{C})$ distributions for the CEMP-s/rs and CEMP-no stars require further detailed investigation, and larger samples are clearly desirable. However, on its face, it appears that reference to carbon plateaus or bands is no longer a valid description of the observations - the morphology of the $A(\mathrm{C})-[\mathrm{Fe} / \mathrm{H}]$ space is much more rich and complex. In retrospect, it is perhaps not surprising that the observed $A(\mathrm{C})$ associated with mass-transfer objects, such as the CEMP-s/rs stars, and that associated with the (presumably) high-mass stellar progenitors of CEMP-no stars should differ so dramatically from one another, as they arise from greatly contrasting nucleosynthetic pathways. Even among the CEMP-no stars themselves, the rather striking differences in $A(\mathrm{C})$ vs. $[\mathrm{Fe} / \mathrm{H}]$ for the Group II and Group III stars calls out for an astrophysical interpretation. It seems plausible that different classes of progenitors, such as the proposed faint mixing-and-fallback supernovae and/or massive ultra low-metallicity spinstars, or other yet-to-be suggested sites, may be able to account for the contrasting behaviors that are observed.

The $A(C)$ distribution of the CEMP-s/rs stars in Group I suggests a single class of progenitors, with no obvious dependence on $[\mathrm{Fe} / \mathrm{H}]$, based on data presently in hand. We note that the $A(\mathrm{C})$ distribution of the CEMP- $r / s$ stars exhibits no clear difference from that of the CEMP-s stars. Better understanding of the underlying processes and their astrophysical implications requires detailed analyses of the full abundance patterns for these stars, more complete knowledge of their binary status (and orbital parameters), and, in particular, more theoretical population-synthesis modeling along the lines of Abate et al. (2015b). We defer a more thorough discussion of the abundance patterns of these stars to future work. 

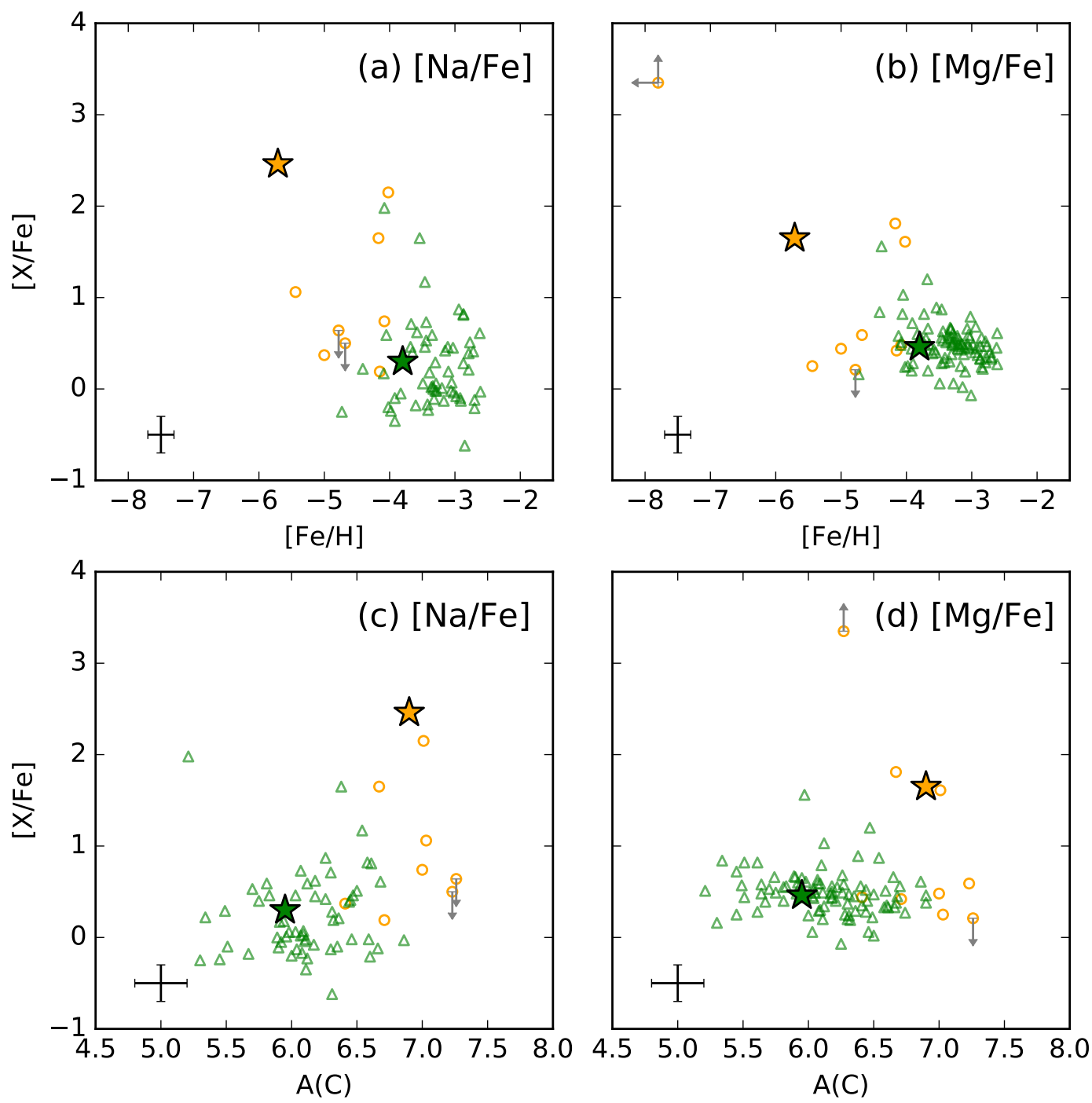

Figure 3. Panels (a) and (b): Distribution of the abundance ratios $[\mathrm{Na} / \mathrm{Fe}]$ and $[\mathrm{Mg} / \mathrm{Fe}]$, as a function of $[\mathrm{Fe} / \mathrm{H}]$. Panels (c) and (d): The same ratios, as a function of $A(\mathrm{C})$. The open green triangles and orange circles represent Group II and Group III stars, respectively. The filled green and orange stars are the well-studied CEMP-no stars BD+44 493 and HE 1327-2326, which we take as type examples of Group II and Group III CEMP-no stars, respectively. Note that only stars with available classifications, based on the $[\mathrm{Ba} / \mathrm{Fe}]$ criterion, are shown. A typical observational error bar is shown in the bottom left of each panel.

The CEMP-no stars of Groups II and III present several compelling behaviors. The clear difference in the $A(\mathrm{C})$ vs. $[\mathrm{Fe} / \mathrm{H}]$ distributions for these two groups has been described above. Here, we examine whether contrasts in the observed abundances for other elements might exist.

It has been previously noticed that a substantial fraction of the extremely metal-poor stars with $[\mathrm{Fe} / \mathrm{H}]$ $<-3.0$ exhibit moderate to strong enhancements in their light-element abundance ratios, such as $[\mathrm{Na} / \mathrm{Fe}]$, $[\mathrm{Mg} / \mathrm{Fe}],[\mathrm{Al} / \mathrm{Fe}]$, and $[\mathrm{Si} / \mathrm{Fe}]$; this "light-element signa- ture" appears to be ubiquitous among the lowest metallicity stars (e.g., Aoki et al. 2002a; Christlieb et al. 2004; Frebel et al. 2005; Aoki et al. 2006; Frebel et al. 2008; Norris et al. 2013). We have examined the behavior of the two most commonly reported light elements, $\mathrm{Na}$ and $\mathrm{Mg}$, for the CEMP-no stars in Groups II and III with this information available, listed in Table 2.

The distributions of the abundance ratios $[\mathrm{Na} / \mathrm{Fe}]$ and $[\mathrm{Mg} / \mathrm{Fe}]$ for the Group II stars (green open triangles) and Group III stars (orange open circles), as functions of $[\mathrm{Fe} / \mathrm{H}]$ and $A(\mathrm{C})$, are shown in Figure 3. This fig- 

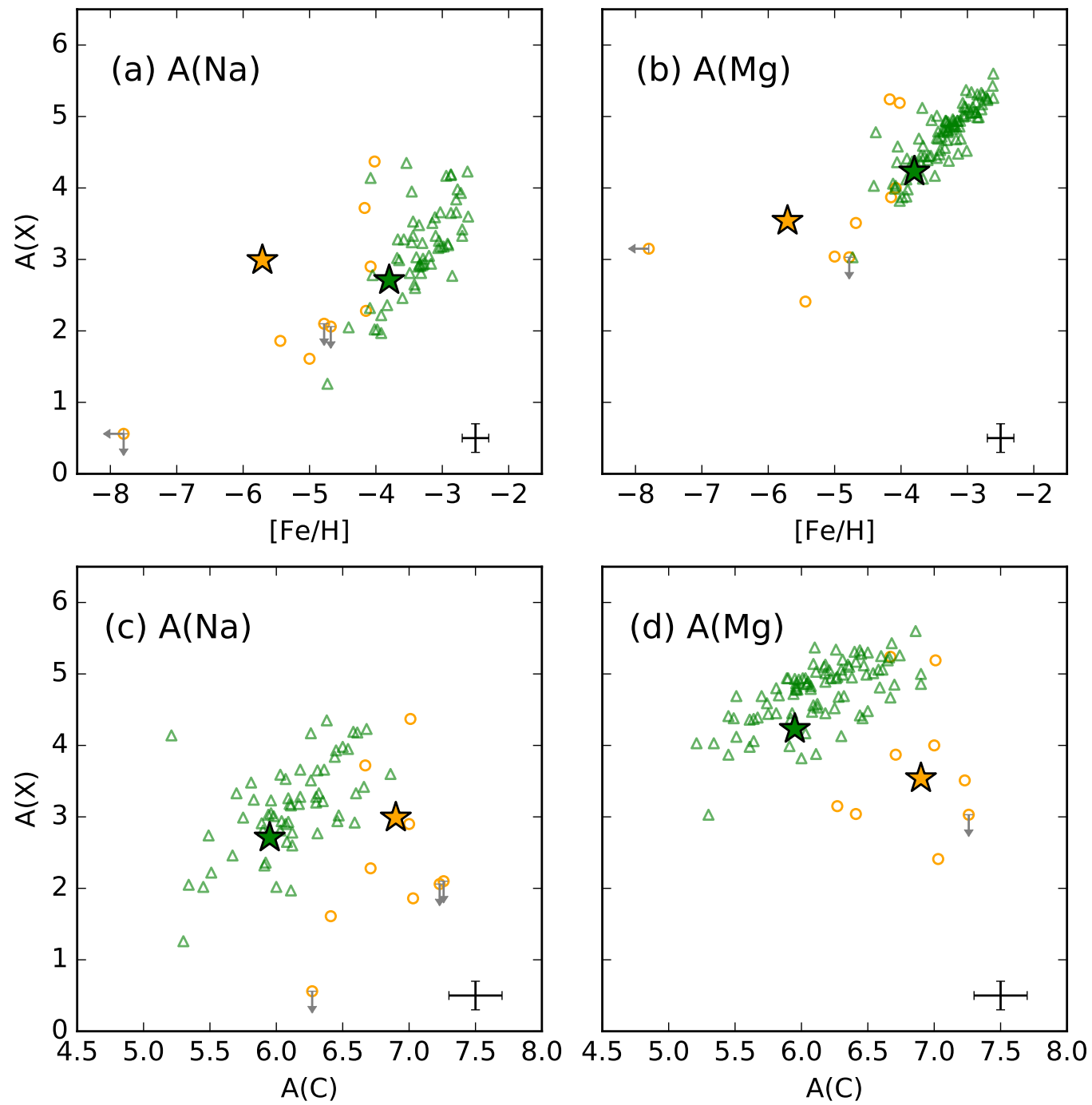

Figure 4. Panels (a) and (b): Distribution of the absolute abundances of $\mathrm{Na}$ and $\mathrm{Mg}, A(\mathrm{Na})$ and $A(\mathrm{Mg})$, as a function of $[\mathrm{Fe} / \mathrm{H}]$. Panels (c) and (d): The same absolute abundances, as a function of $A(\mathrm{C})$. The colors and symbols are the same as in Figure 3. Note that only stars with available classifications, based on the $[\mathrm{Ba} / \mathrm{Fe}]$ criterion, are shown. A typical observational error bar is shown in the bottom right of each panel.

ure highlights the location of two stars, BD+44 493 and HE 1327-2326, as filled green and orange stars, respectively. We consider these stars as canonical examples of Group II and III CEMP-no stars, respectively. The previously noted presence of enhancement in these ratios, with respect to $[\mathrm{Fe} / \mathrm{H}]$, is apparent in the upper panels in Figure 3. Many of the stars with $[\mathrm{Fe} / \mathrm{H}]<-4.0$ exhibit over-abundances of $\mathrm{Na}$ and $\mathrm{Mg}$, although some do not. The largest enhancements are found for the Group III stars, although there are also a number of Group II stars with this signature present as well. The lower pan- els show these same ratios with respect to $A(\mathrm{C})$. Enhancements in $[\mathrm{Na} / \mathrm{Fe}]$ and $[\mathrm{Mg} / \mathrm{Fe}]$ for stars with $A(\mathrm{C})$ $\gtrsim 6.5$ can be seen, where once again the largest overabundances are found for the Group III stars, along with a few exceptional Group II stars.

Figure 4 shows the distributions of absolute abundances, $A(\mathrm{Na})$ and $A(\mathrm{Mg})$, as functions of $[\mathrm{Fe} / \mathrm{H}]$ and $A(\mathrm{C})$. There is no clear separation of the absolute abundances of $\mathrm{Na}$ and $\mathrm{Mg}$ for Group II and Group III stars seen in panels (a) and (b); both scale roughly with $[\mathrm{Fe} / \mathrm{H}]$. However, when these same absolute abundances 
are plotted vs. $A(\mathrm{C})$, shown in panels (c) and (d), an apparent dichotomy emerges - the Group II stars scale roughly with $A(\mathrm{C})$, while the Group III stars exhibit no correlation with $A(\mathrm{C})$, but are instead offset to generally lower values for $A(\mathrm{C}) \gtrsim 6.0$, with only a few exceptions. Based on work in progress, we note that there exist similar behaviors in the $A(\mathrm{Al})-A(\mathrm{C})$ and $A(\mathrm{Si})-A(\mathrm{C})$ spaces as well. The two Group III stars with relatively high $A(\mathrm{Na})$ and $A(\mathrm{Mg})$ seen in the lower panels (HE 10121540: $[\mathrm{Fe} / \mathrm{H}]=-4.17, A(\mathrm{C})=6.67,[\mathrm{Na} / \mathrm{Fe}]=+1.65$, $[\mathrm{Mg} / \mathrm{Fe}]=+1.81$, and HE 2139-5432: $[\mathrm{Fe} / \mathrm{H}]=-4.02$, $A(\mathrm{C})=7.01,[\mathrm{Na} / \mathrm{Fe}]=+2.15,[\mathrm{Mg} / \mathrm{Fe}]=+1.61)$ are selected near the extremum of the region identified with this group shown in Figure 1, close to the locations of the Group II stars. These two stars also present behaviors that are more similar to Group II stars in all panels of Figures 3 and 4; their identification as Group III stars (which is somewhat arbitrary at this early stage of understanding) is perhaps suspect.

Based on the behaviors noted above, we argue that it is likely that more than one class of progenitors for the CEMP-no stars of Groups II and III exist. To our knowledge, there is no clear predicted signature that differentiates between the two most frequently considered progenitor models for CEMP-no stars. However, the two canonical Group II and Group III stars we have highlighted above have indeed been associated by previous authors as type examples of the faint mixingand-fallback supernovae models (BD+44 493; e.g., Ito et al. 2013; Placco et al. 2014c; Roederer et al. 2014b, 2016) and spinstar models (HE 1327-2326; e.g., Maeder \& Meynet 2015; Maeder et al. 2015). We note, however, that the observed elemental abundance distribution of HE 1327-2326 has also been well-fit to a faint supernova model (Iwamoto et al. 2005; Tominaga et al. 2007), although the models considered under-produce its observed $[\mathrm{N} / \mathrm{Fe}]$ and do not predict its $[\mathrm{Sr} / \mathrm{Fe}]$ abundance.

It may be premature to associate Group II stars with faint mixing-and-fallback SNe progenitors $\left(15-40 \mathrm{M}_{\odot}\right.$; Nomoto et al. 2013) and the Group III stars with spinstar progenitors ( $\gtrsim 50 \mathrm{M}_{\odot}$; Meynet et al. 2006), and we remain open to the possibility that other classes of progenitors may contribute in the early chemical history of the Universe ${ }^{10}$. Further theoretical development and modeling of the environments (e.g., the amount of baryonic mass available for mixing and dilution of the yields from both classes of model progenitors, expanding on

\footnotetext{
${ }^{10}$ Neutron-capture abundance patterns found for a few of the CEMP-no Group II stars indicate a small amount of $r$-process material is present (Roederer et al. 2014a), which is not predicted to be produced by mixing-and-fallback SNe.
}

the initial efforts of, e.g., Cooke \& Madau 2014; Susa et al. 2014) is highly encouraged.

\subsection{Single Stars vs. Binary Stars}

Knowledge of a given CEMP star's binary status is crucial for confident identification of its likely source of carbon enrichment, whether extrinsic (AGB binary mass-transfer) or intrinsic (the star is born in a previously C-enhanced environment).

Previous investigations have shown that a high fraction of the CEMP-s stars are in binary systems (e.g., Lucatello et al. 2005b; Starkenburg et al. 2014; Jorissen et al. 2016, and references therein), prompting the suggestion that all CEMP-s/rs stars have binary companions. Most recently, Hansen et al. (2016c) report that, although $82 \pm 26 \%$ (18 of 22$)^{11}$ of their sample of CEMP$s / r s$ stars are indeed binaries, there remain a small fraction $(18 \pm 10 \% ; 4$ of 22$)$ of stars that, despite a long-term campaign of precision radial-velocity measurements, appear to be single (or binaries with extremely long periods). As pointed out by these authors, the existence of this handful of "anomalous" C- and Ba-enhanced single stars opens the door to the possibility of enrichment by massive stars capable of producing $\mathrm{Ba}$ (and perhaps other heavy elements) via a weak $s$-process (see, e.g., Frischknecht et al. 2016, and references therein).

Hansen et al. (2016b) also obtained precision longterm radial-velocity monitoring observations of a sample of CEMP-no stars, reporting that the binary fraction of CEMP-no stars is $17 \pm 9 \%(4 \text { of } 24)^{12}$, i.e., no different than the observed binary fraction of C-normal giants in the halo (16 $44 \%$; Carney et al. 2003). It is clear that these two sub-classes of CEMP stars have different binary status, with implications for the nature of their progenitors.

Figure 5 shows the distribution of $A(\mathrm{C})$ as a function of $[\mathrm{Fe} / \mathrm{H}]$, for CEMP stars with known (or likely, meaning that further confirmation is desired) binary status, including 35 CEMP-s/rs stars and 22 CEMP-no stars (Dearborn et al. 1986; McClure \& Woodsworth 1990; Preston \& Sneden 2001; Thompson et al. 2008; Spite et al. 2013; Placco et al. 2015; Hansen et al. 2016b,c;

\footnotetext{
11 The published error bar on the CEMP-s fraction in Hansen et al. 2016c of $10 \%$ is formally incorrect, and should be substantially higher, $26 \%$, when calculated from traditional error propagation, which we have used here. However, it should also be recognized that fractions of binary/non-binary stars for a given population are in fact correlated, in the sense that their sum remains fixed, hence improved statistical treatment of the appropriate error bars, rather than assigning errors from strictly Poisson statistics, needs to be considered in the future.

12 Three stars (CS 22166-016, CS 29527-015, and CS 22878027) were excluded in our calculations here, since the available high-resolution data is not able to clearly classify them as CEMP stars.
} 


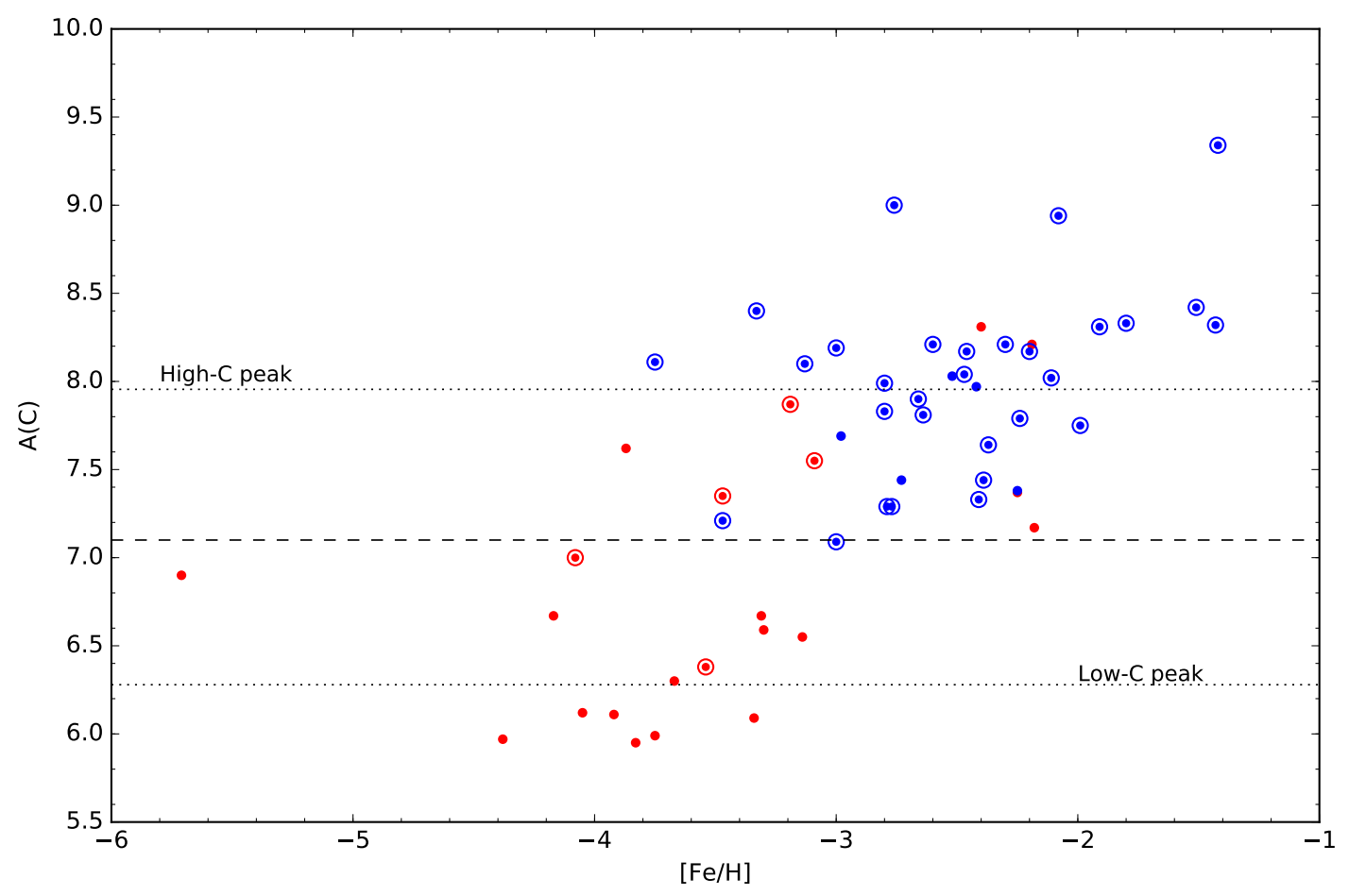

Figure 5. The distribution of $A(\mathrm{C})$, as a function of $[\mathrm{Fe} / \mathrm{H}]$, of the known (and likely) binaries and known (and likely) single stars in our compiled sample of CEMP stars. As before, the blue and red colors indicate the CEMP-s/rs and CEMP-no stars, respectively. The dots $(\cdot)$ represent the known/likely single stars; the small dots inside the larger open circles $(\odot)$ represent the known/likely binary stars. The dotted black lines indicate the location of the two $A(\mathrm{C})$ peaks described in the text. The dashed black line is a fiducial at $A(\mathrm{C})=7.1$, placed at the mid-point of the peaks in the marginal histograms of $A(\mathrm{C})$ shown in Figure 1; see text for further discussion.

Jorissen et al. 2016). The dots in this figure represent single stars, while the binary stars are shown as a dot inside a large open circle. The binary status of these stars, where known, is also listed in Table 1. Although many CEMP stars do not yet have observational constraints on their binary nature, the information in hand does provide the basis for a number of interesting interpretations.

Most CEMP-s/rs stars (86 $\pm 21 \%$; 30 of 35) shown in Figure 5 are recognized binaries, and the majority $(77 \pm 25 \% ; 17$ of 22$)$ of the CEMP-no stars are single stars. We note in passing that it is remarkable how well the division of the stars by the fiducial line at $A(\mathrm{C})$ $=7.1$, based on the marginal histogram of $A(\mathrm{C})$ from Figure 1, and discussed further below, effectively separates the binary nature of all of the CEMP-s/rs stars and most of the CEMP-no stars. This clear contrast of the binary fraction between the two sub-classes strongly supports the hypothesis that the over-abundance in carbon and $s$-process elements for most CEMP-s/rs stars is likely extrinsic, while the carbon enhancement in most CEMP-no stars is the result of an intrinsic process. Therefore, the scatter in $A(\mathrm{C})$ among the CEMP-s/rs stars shown in Figure 1 might be explained by masstransfer models of AGB stars with differing masses, mass-transfer efficiencies, and possible post-transfer dilution (e.g., Abate et al. 2015c, and references therein). Further theoretical work is required, of course, in order to establish if the observed distribution of $A(\mathrm{C})$ can be captured by extant models, or whether additional complexity must be introduced. Regardless, the sample of CEMP-s/rs stars with measured $A(\mathrm{C})$ is now sufficiently large that such constraints should be feasible.

There are a number of interesting exceptions to the rule found among the stars in our sample. For example, there are several CEMP-s/rs stars located in the low-C region in Figure 1 (or in the transition region between the two regions), whose binary status are unfortunately unknown. Accounting for their low $A(\mathrm{C})$, coupled with high $[\mathrm{Ba} / \mathrm{Fe}]$ ratios, remains a challenge. In addition, the five apparently single CEMP-s/rs stars in the high$\mathrm{C}$ region of Figure 5 should yield interesting constraints on the source of their carbon and barium enrichment.

While the great majority of CEMP-no stars with known binary status are single stars, there are five CEMP-no stars in our sample that are recognized bina- 

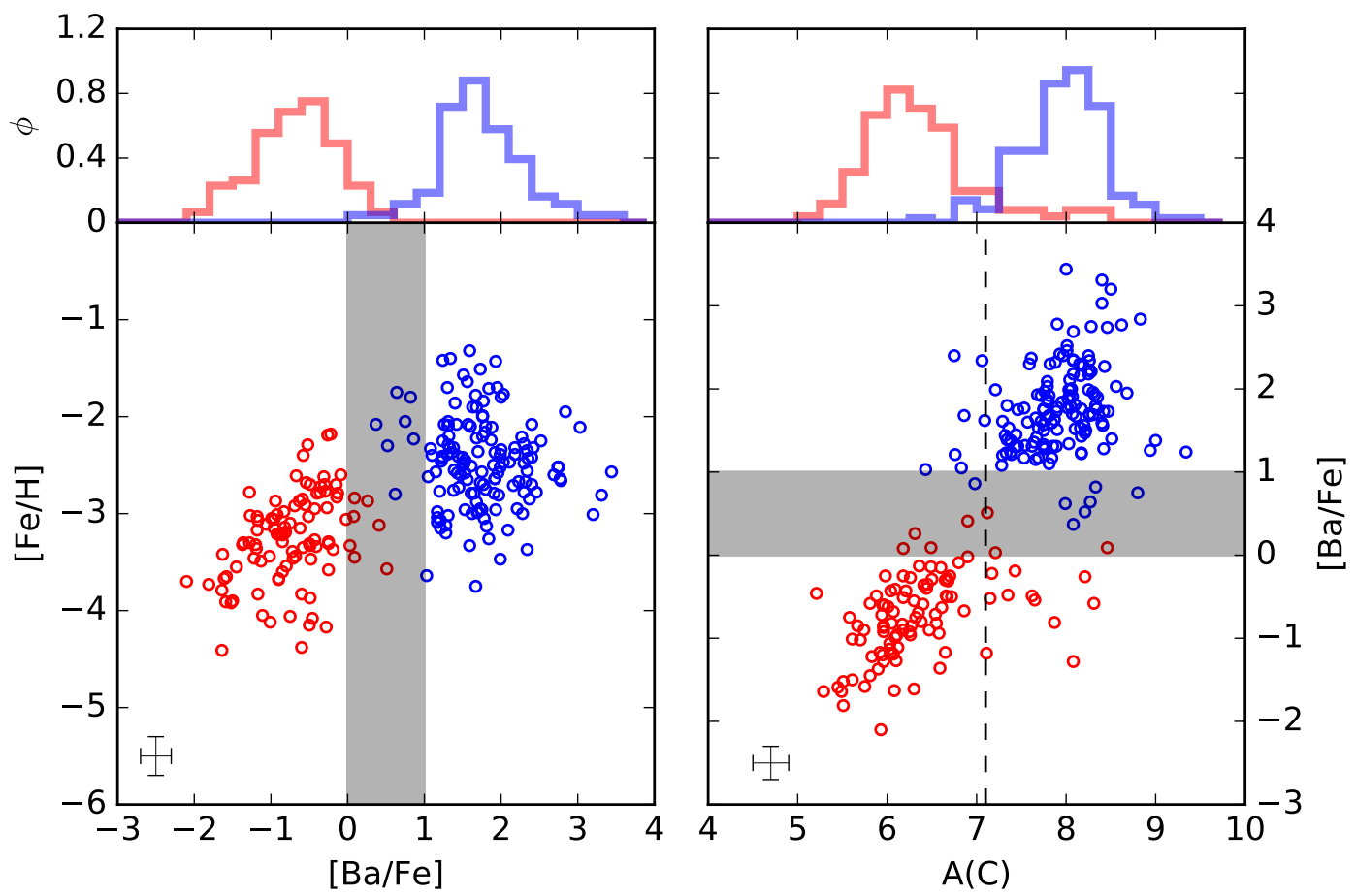

Figure 6. The distribution of $[\mathrm{Fe} / \mathrm{H}]$, as a function of $[\mathrm{Ba} / \mathrm{Fe}]$ (left panels), and $[\mathrm{Ba} / \mathrm{Fe}]$, as a function of $A(\mathrm{C})$ (right panels). As before, the blue and red colors indicate the CEMP-s/rs and CEMP-no stars, respectively. The gray shaded region in both panels represents stars with intermediate $[\mathrm{Ba} / \mathrm{Fe}]$ ratios, $0.0<[\mathrm{Ba} / \mathrm{Fe}]<+1.0$, which complicates their sub-classification. The dashed vertical line in the right panel is a fiducial at $A(\mathrm{C})=7.1$, placed between the peaks of the histograms of the $A(\mathrm{C})$ distributions for Group I and Group II and III stars shown in Figure 1 . There are 15 stars with upper limits on [Ba/Fe] $<0.0$ not shown in this figure. A typical observational error bar is shown in the bottom left of each panel.

ries. Among these, three of the CEMP-no binaries are located in the high-C region, while two CEMP-no binaries fall in the low- $\mathrm{C}$ region. As mentioned in Section 3 above, there are also several CEMP-no stars located in the high- $\mathrm{C}$ region but without known binary status. It is indeed crucial to conduct further radial-velocity monitoring of these exceptional stars, as well as to determine their full elemental-abundance patterns, in order to better constrain their origin.

\subsection{Interesting Outliers}

We have noted a number of stars in our sample that can be considered outliers, in the sense that they deviate in some manner from the bulk of stars with similar sub-classifications and/or binary status. Exploration of the numerous and varied possibilities to account for the underlying causes of the recognized deviant cases is well beyond the scope of this paper. However, for convenience, and to spawn their future detailed study, Table 3 lists the recognized outliers, and their properties - $[\mathrm{Fe} / \mathrm{H}], A(\mathrm{C}),[\mathrm{C} / \mathrm{Fe}]_{c},[\mathrm{Ba} / \mathrm{Fe}],[\mathrm{Eu} / \mathrm{Fe}]$, classification and binarity status (1 indicates known single stars, 2 indicates known binaries) - grouped along with stars of similar behavior, as well as stars without known binary status but with disparate $A(\mathrm{C})$ and $[\mathrm{Ba} / \mathrm{Fe}]$.

\subsection{Is $A(C)$ a more Fundamental Indicator of CEMP Classification?}

Although the CEMP stars exhibit complex behaviors in the $A(\mathrm{C})-[\mathrm{Fe} / \mathrm{H}]$ space, there is indeed a clear separation between the CEMP-s/rs and CEMP-no stars, as seen in the marginal histogram of $A(\mathrm{C})$ shown in Figure 1. This separation is observed in Figure 6 as well. The left panels of Figure 6 show the distribution of $[\mathrm{Fe} / \mathrm{H}]$ and $[\mathrm{Ba} / \mathrm{Fe}]$, along with the marginal histogram of $[\mathrm{Ba} / \mathrm{Fe}]$ for both the CEMP-r/rs and CEMPno stars, plotted at the top. The shaded region with 0.0 $<[\mathrm{Ba} / \mathrm{Fe}]<+1.0$ was not considered in the original definition of the two sub-classes (Beers \& Christlieb 2005), and later studies adopted different criteria for the subclassification of stars in this region.

The right panels of Figure 6 show the relation between $[\mathrm{Ba} / \mathrm{Fe}]$ and $A(\mathrm{C})$, along with the marginal histogram of $A(\mathrm{C})$ plotted at the top. There exists a clear correlation between $A(\mathrm{C})$ and $[\mathrm{Ba} / \mathrm{Fe}]$. The vertical line represents a fiducial at $A(\mathrm{C})=7.1$, placed at the midpoint of the two $A(\mathrm{C})$ peaks. Although there exists an overlap between the stars sub-classified on the basis of $[\mathrm{Ba} / \mathrm{Fe}]$, 
there is a clear separation between the majority of stars in the two sub-classes.

Among the interesting outliers described in the previous subsection (and shown in Figure 5), three of the eight CEMP-no stars with $A(\mathrm{C})>7.1$ are known binaries, while five are known single stars, double the expected binary fraction based on that of most CEMP-no stars according to Hansen et al. (2016b); further speculation is not yet warranted, given the large Poisson errors in this fraction. The apparently disparate stars shown in the right panel of Figure 6 are of interest as well. There is only one known binary among the CEMP-s/rs stars in the upper left quadrant of the right panel of this figure $(A(\mathrm{C}) \leq 7.1$ and $[\mathrm{Ba} / \mathrm{Fe}]>+1.0)$, while three of the nine CEMP-no stars in the lower-right quadrant $(A(\mathrm{C})$ $>7.1$ and $[\mathrm{Ba} / \mathrm{Fe}]<0)$ are known binaries. Future radial-velocity monitoring of these exceptional cases is clearly of interest.

The original definition of the CEMP sub-classes was purely empirical, based on a dichotomy noticed in the distribution of $[\mathrm{Ba} / \mathrm{Fe}]$ as a function of $[\mathrm{Fe} / \mathrm{H}]$ (e.g., Aoki et al. 2002b; Ryan et al. 2005). Beers \& Christlieb (2005) quantified this dichotomy, which has been widely adopted since. However, barium abundance measurements can only be readily obtained from moderatelyhigh to high-resolution spectroscopy, which for fainter stars $(V>14)$ generally requires large telescope time. In contrast, C-abundance estimates can be obtained from low- to medium-resolution spectroscopy, which can be readily acquired by smaller telescopes, such as the 2.5m telescope used for the Sloan Digital Sky Survey (SDSS, York et al. 2000; Lee et al. 2013). The clear separation between the CEMP-s/rs and CEMP-no stars based on $A(\mathrm{C})$ we have noted in this study can thus greatly expedite the process of sub-classification.

In addition, the degree of enhancement in carbon abundance is likely to depend primarily on the nature (and mass) of the progenitors (although dilution in the parent mini-halo may also play an important role); large enhancements of $A(\mathrm{C})$ by mass transfer from low- to intermediate-mass AGB stars and lower enhancements in the natal clouds of high-mass progenitors. Barium enhancement can arise from a variety of processes, e.g., the $r$-process at low metallicity (from progenitors of a currently uncertain nature; Roederer et al. 2014a), and the weak $s$-process in massive stars (Frischknecht et al. 2016), as well as from AGB nucleosynthesis (Bisterzo et al. 2011). Therefore, we suggest that the absolute carbon abundance, $A(\mathrm{C})$, may be a more fundamental criterion for separating the CEMP-s/rs stars from CEMP-no stars.

In order to assess the efficacy of sub-classification for CEMP stars based on $A(\mathrm{C})$, we have re-classified the stars in our sample - stars with $A(\mathrm{C}) \leq 7.1$ are classi- fied as CEMP-no stars and those with $A(\mathrm{C})>7.1$ are considered CEMP-s/rs stars. The left-hand panels of Figure 7 are obtained from application of the conventional classification criterion, based on $[\mathrm{Ba} / \mathrm{Fe}]$ (Beers \& Christlieb 2005). The right-hand panels show the reclassified CEMP stars, obtained from application of the suggested $A(\mathrm{C})$ criterion. Both classifications are listed in column (10) of Table 1.

As can be verified from inspection of Figure 7, the distinctive separation of these two sub-classes of CEMP stars based on $A(\mathrm{C})$ is apparently as effective as that obtained from the application of the conventional $[\mathrm{Ba} / \mathrm{Fe}]$ criterion. Employing the $A(\mathrm{C})$ criterion, our sample of stars includes 159 CEMP-s/rs stars and 115 CEMP-no stars. Of the 159 re-classified CEMP-s/rs stars, 20 were originally classified as CEMP-no stars, a "success rate" (if the conventional classification approach is taken as ground truth) of $87 \%$. For the 115 re-classified CEMP-no stars, 8 stars were classified as CEMP-s/rs stars by the traditional criterion, a success rate of $93 \%$. The binary fractions of the re-classified CEMP-s/rs and CEMP-no stars changed from $86 \pm 21 \%$ (30 of 35 ) to $76 \pm 18 \%$ (32 of 42 ) and $23 \pm 11 \%$ ( 5 of 22 ) to $20 \pm 13 \%$ (3 of 15 ), respectively, which are not significant, given the large Poisson errors. We have applied the $A(\mathrm{C})$ criterion to the 31 unclassified CEMP stars listed at the end of Table 1 - those either without reported $[\mathrm{Ba} / \mathrm{Fe}]$ abundance ratios or located in the ambiguous zone $(0.0<[\mathrm{Ba} / \mathrm{Fe}]<+1.0)$. There are an additional 19 CEMP-no stars and 12 CEMP-s/rs stars based on the new classification scheme.

\section{CONCLUSIONS}

We have investigated the absolute carbon-abundance distribution of CEMP stars based on a reasonably complete compilation of available high-resolution spectroscopic data. The $A(\mathrm{C})$ distribution of the CEMP stars clearly exhibits (at least) a bimodality, as has been noted by a number of previous authors. However, there exist complex behaviors embedded in the $A(\mathrm{C})-[\mathrm{Fe} / \mathrm{H}]$ space, not easily captured by description as plateaus or bands; we suggest use of the terms high-C and low-C regions. We separate CEMP stars into three groups - Group I, comprising primarily CEMP-s/rs stars, and Group II and Group III comprising CEMP-no stars. Along with the apparent dichotomy in the absolute abundance distribution of $\mathrm{Na}$ and $\mathrm{Mg}$ as a function of $A(\mathrm{C})$ for the CEMP-no stars, we suggest this provides the first clear observational evidence for the existence of multiple progenitor populations of the CEMP-no stars in the early Universe.

Based on the known binary status for a subset of the 

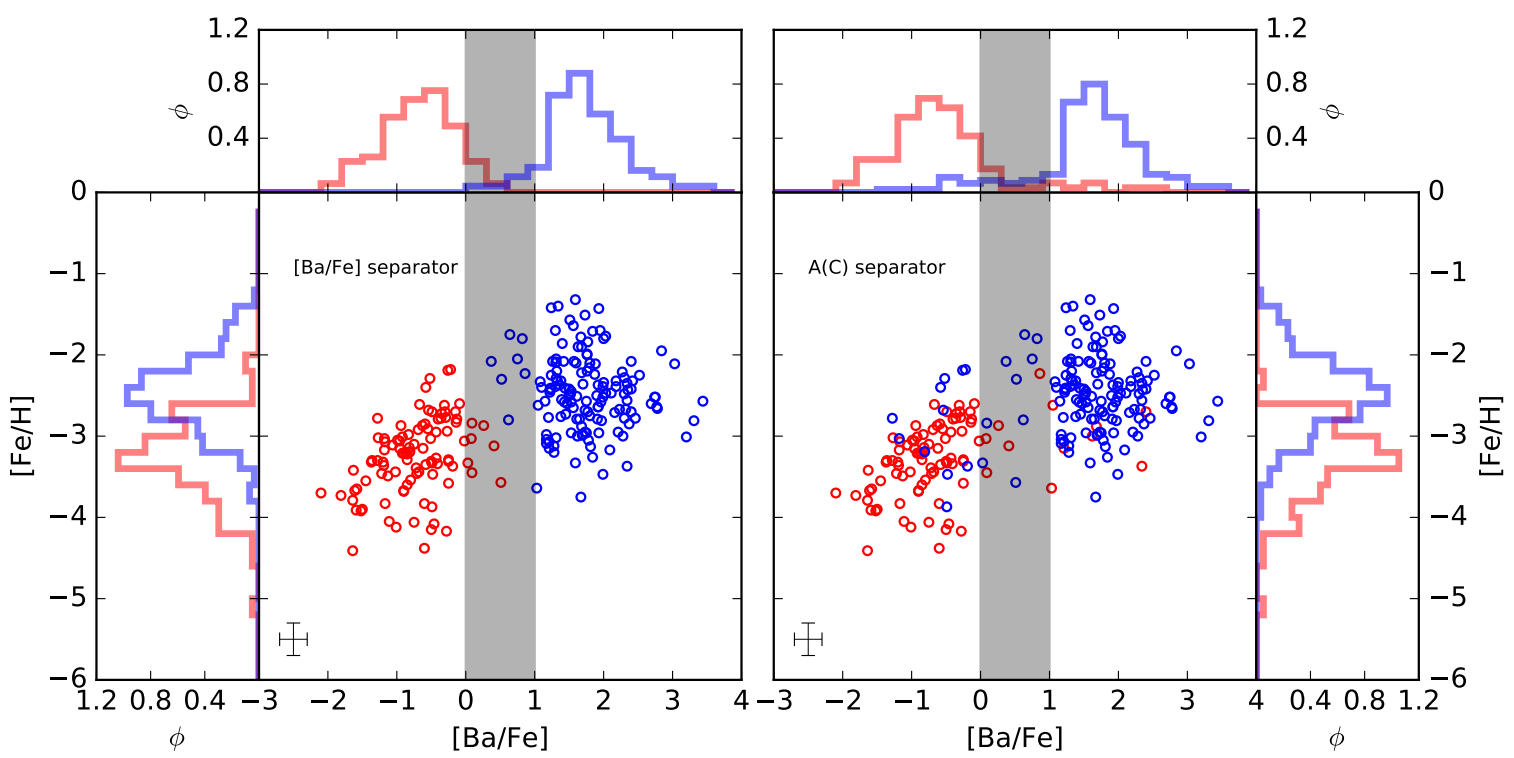

Figure 7. Comparison between stars sub-classified into the CEMP-s/rs and CEMP-no categories using either the [Ba/Fe] or the $A(\mathrm{C})$ criterion. As before, the blue and red colors indicate the CEMP-s/rs and CEMP-no stars, respectively. The left panels correspond to use of the $[\mathrm{Ba} / \mathrm{Fe}]$ criterion, while the right panels are based on the $A(\mathrm{C})$ criterion. That is, CEMP-no stars are classified as such if they have $A(\mathrm{C}) \leq 7.1$, while CEMP-s/rs stars are classified as such if they have $A(\mathrm{C})>7.1$. There are 15 stars with upper limits on $[\mathrm{Ba} / \mathrm{Fe}]<0.0$ not shown in this figure. A typical observational error bar is shown in the bottom left of each panel.

CEMP stars, we strongly support the hypothesis that the carbon enhancement of the CEMP-s/rs stars is extrinsic, and due to mass-transfer of material enriched by an AGB companion, while the carbon enhancement of CEMP-no stars is intrinsic, and due to enrichment of their natal clouds by high-mass progenitor stars. According to this view, the CEMP-no stars are bona-fide second generation stars, as supported by other lines of evidence (see summary in Hansen et al. 2016b).

We have identified a number of interesting outliers, worthy of further exploration, that differ from the general behaviors of otherwise similar stars in either their binary status or with disparate $A(\mathrm{C})$ and $[\mathrm{Ba} / \mathrm{Fe}]$ ratios.

Finally, we have presented evidence that the separation of CEMP-s/rs stars from CEMP-no stars can be accomplished as well (or better) using the simple criterion $A(\mathrm{C})>7.1$ for $\mathrm{CEMP}-s / r s$ stars and $A(\mathrm{C}) \leq 7.1$ for CEMP-no stars. As $A(\mathrm{C})$ can be obtained from low- to medium-resolution spectroscopy, rather than the highresolution spectroscopy required for the former criterion based on $[\mathrm{Ba} / \mathrm{Fe}]$ (and $[\mathrm{Ba} / \mathrm{Eu}]$ ) ratios, this provides an efficient means to quickly isolate the most interesting CEMP sub-classes in massive spectroscopic surveys now and in the future. Given the multiple nucleosynthetic pathways for the production of $\mathrm{Ba}$ in the early Universe known (or suggested) to exist, we assert that the $A(\mathrm{C})$ criterion may also be more astrophysically fundamental.

We plan to employ the $A(\mathrm{C})$ sub-classification approach to the thousands of CEMP stars presently identi- fied by SDSS/SEGUE and other large surveys from the past, e.g., the HK survey (Beers et al. 1985, 1992) and the Hamburg/ESO survey (Christlieb 2003), enabling consideration of potential differences in the spatial and kinematic distributions of CEMP-s/rs and CEMP-no stars (e.g., Carollo et al. 2014), as well as in their relative frequencies as a function of $[\mathrm{Fe} / \mathrm{H}]$ (e.g., Lee et al. 2013). Comparison of these observables with the predictions of modern Galactic chemical evolution models (e.g., Côté et al. 2016; Crosby et al. 2016) should prove illuminating.

Future progress requires a significant increase in the numbers of CEMP-s/rs stars and CEMP-no stars with available high-resolution spectroscopy, so that their full elemental-abundance distributions can be considered in more detail (both from the ground and in the near-UV from space), and used to better constrain their likely progenitors. This goal is being actively pursued. Additional long-term radial-velocity monitoring of CEMP stars is also likely to pay substantial scientific dividends. For both reasons, the identification of, in particular, bright CEMP stars is being given high priority in our ongoing survey efforts.

There is also a clear need for additional development of theory and modeling, to obtain deeper understanding of the nucleosynthesis processes in operation in the suggested progenitors of CEMP stars. Links between the CEMP phenomenon and the early-Universe initial mass function have been considered by a number of pre- 
vious authors (e.g., Lucatello et al. 2005a; Tumlinson 2007a,b; Suda et al. 2013; Carollo et al. 2014; Lee et al. 2014), based on the relative frequencies of CEMP-s/rs and CEMP-no stars in the halo system. Here we have shown that this idea might be extended by taking into account the presumed differences in the masses of the progenitors of the Group II and Group III CEMP-no stars.

The authors thank C. Abate, W. Aoki, A. Maeder, G. Meynet, M. Pignatari, R. Stancliffe, N. Tominaga, and K. Venn for useful discussions, as well as the anonymous referee for providing useful comments on the manuscript, which improved this paper. J.Y., T.C.B.,
V.M.P., K.C.R., D.C., and I.U.R. acknowledge partial support from grant PHY 14-30152; Physics Frontier Center/JINA Center for the Evolution of the Elements (JINA-CEE), awarded by the US National Science Foundation. S.H. participated this work as an REU student at the University of Notre Dame. T.T.H. acknowledges partial support from grant AST-1108811, awarded by the US National Science Foundation.

This research made use of NASA's Astrophysics Data System, the SIMBAD astronomical database, operated at CDS, Strasbourg, France, and the SAGA database (Suda et al. 2008, 2011; Yamada et al. 2013) (http://sagadatabase.jp). This work also made extensive use of python.org, astropy.org, and scikit-learn.org.

\section{REFERENCES}

Abate, C., Pols, O. R., Izzard, R. G., \& Karakas, A. I. 2015a, A\&A, 581, A22

Abate, C., Pols, O. R., Izzard, R. G., Mohamed, S. S., \& de Mink, S. E. 2013, A\&A, 552, A26

Abate, C., Pols, O. R., Karakas, A. I., \& Izzard, R. G. 2015b, A\&A, 576, A118

Abate, C., Pols, O. R., Stancliffe, R. J., et al. 2015c, A\&A, 581, A62

Abate, C., Stancliffe, R. J., \& Liu, Z.-W. 2016, A\&A, 587, A50

Allen, D. M., Ryan, S. G., Rossi, S., Beers, T. C., \&

Tsangarides, S. A. 2012, A\&A, 548, A34

Aoki, W., Beers, T. C., Christlieb, N., et al. 2007, ApJ, 655, 492

Aoki, W., Norris, J. E., Ryan, S. G., Beers, T. C., \& Ando, H. 2002a, ApJL, 576, L141

Aoki, W., Ryan, S. G., Norris, J. E., et al. 2002b, ApJ, 580, 1149

Aoki, W., Frebel, A., Christlieb, N., et al. 2006, ApJ, 639, 897

Aoki, W., Beers, T. C., Sivarani, T., et al. 2008, ApJ, 678, 1351

Aoki, W., Beers, T. C., Lee, Y. S., et al. 2013, AJ, 145, 13

Asplund, M., Grevesse, N., Sauval, A. J., \& Scott, P. 2009, ARA\&A, 47, 481

Barklem, P. S., Christlieb, N., Beers, T. C., et al. 2005, A\&A, 439, 129

Beers, T. C., \& Christlieb, N. 2005, ARA\&A, 43, 531

Beers, T. C., Preston, G. W., \& Shectman, S. A. 1985, AJ, 90, 2089

-. 1992, AJ, 103, 1987

Beers, T. C., Sivarani, T., Marsteller, B., et al. 2007, AJ, 133, 1193

Bessell, M. S., Collet, R., Keller, S. C., et al. 2015, ApJL, 806, L16

Bisterzo, S., Gallino, R., Straniero, O., Cristallo, S., \& Käppeler, F. 2011, MNRAS, 418, 284

Bonifacio, P., Caffau, E., Spite, M., et al. 2015, A\&A, 579, A28

Carney, B. W., Latham, D. W., Stefanik, R. P., Laird, J. B., \& Morse, J. A. 2003, AJ, 125, 293

Carollo, D., Freeman, K., Beers, T. C., et al. 2014, ApJ, 788, 180

Chiappini, C. 2013, Astronomische Nachrichten, 334, 595

Christlieb, N. 2003, in Reviews in Modern Astronomy, Vol. 16, Reviews in Modern Astronomy, ed. R. E. Schielicke, 191

Christlieb, N., Beers, T. C., Barklem, P. S., et al. 2004, A\&A, 428, 1027

Cohen, J. G., Christlieb, N., McWilliam, A., et al. 2008, ApJ, 672,320

Cohen, J. G., Christlieb, N., Thompson, I., et al. 2013, ApJ, 778, 56
Cooke, R., Pettini, M., \& Murphy, M. T. 2012, MNRAS, 425, 347

Cooke, R., Pettini, M., Steidel, C. C., Rudie, G. C., \&

Jorgenson, R. A. 2011, MNRAS, 412, 1047

Cooke, R. J., \& Madau, P. 2014, ApJ, 791, 116

Côté, B., Ritter, C., O'Shea, B. W., et al. 2016, ApJ, 824, 82

Crosby, B. D., O'Shea, B. W., Beers, T. C., \& Tumlinson, J. 2016, ApJ, 820, 71

Cui, W. Y., Sivarani, T., \& Christlieb, N. 2013, A\&A, 558, A36

Dardelet, L., Ritter, C., Prado, P., et al. 2015, ArXiv e-prints, arXiv: 1505.05500

Dearborn, D. S. P., Liebert, J., Aaronson, M., et al. 1986, ApJ, 300,314

Fraley, C., \& Raftery, A. E. 2002, JASA, 97, 611

Fraley, C., Raftery, A. E., Murphy, B., \& Scrucca, L. 2012, mclust Version 4 for R: Normal Mixture Modeling for Model-Based Clustering, Classification, and Density Estimation, Technical Report No. 597, Department of Statistics, University of Washington

Frebel, A., Casey, A. R., Jacobson, H. R., \& Yu, Q. 2013, ApJ, 769,57

Frebel, A., Chiti, A., Ji, A. P., Jacobson, H. R., \& Placco, V. M. 2015, ApJL, 810, L27

Frebel, A., Collet, R., Eriksson, K., Christlieb, N., \& Aoki, W. 2008, ApJ, 684, 588

Frebel, A., \& Norris, J. E. 2015, ArXiv e-prints, arXiv:1501.06921

Frebel, A., Aoki, W., Christlieb, N., et al. 2005, Nature, 434, 871

Frischknecht, U., Hirschi, R., Pignatari, M., et al. 2016, MNRAS, 456, 1803

Goswami, A., \& Aoki, W. 2010, MNRAS, 404, 253

Goswami, A., Aoki, W., Beers, T. C., et al. 2006, MNRAS, 372, 343

Goswami, A., Aoki, W., \& Karinkuzhi, D. 2016, MNRAS, 455, 402

Gratton, R. G., Sneden, C., Carretta, E., \& Bragaglia, A. 2000, A\&A, 354, 169

Hampel, M., Stancliffe, R. J., Lugaro, M., \& Meyer, B. S. 2016, ArXiv e-prints, arXiv:1608.08634

Hansen, C. J., Nordström, B., Hansen, T. T., et al. 2016a, A\&A, 588, A37

Hansen, T., Hansen, C. J., Christlieb, N., et al. 2014, ApJ, 787, 162

—. 2015a, ApJ, 807, 173

Hansen, T. T., Andersen, J., Nordström, B., et al. 2016b, A\&A, 586, A160

-. 2016c, A\&A, 588, A3 
-. 2015b, A\&A, 583, A49

Heger, A., \& Woosley, S. E. 2010, ApJ, 724, 341

Herwig, F. 2005, ARA\&A, 43, 435

Herwig, F., Pignatari, M., Woodward, P. R., et al. 2011, ApJ, 727,89

Hollek, J. K., Frebel, A., Placco, V. M., et al. 2015, ApJ, 814, 121

Hollek, J. K., Frebel, A., Roederer, I. U., et al. 2011, ApJ, 742, 54

Ito, H., Aoki, W., Beers, T. C., et al. 2013, ApJ, 773, 33

Iwamoto, N., Umeda, H., Tominaga, N., Nomoto, K., \& Maeda, K. 2005, Science, 309, 451

Jacobson, H. R., Keller, S., Frebel, A., et al. 2015, ApJ, 807, 171

Jonsell, K., Barklem, P. S., Gustafsson, B., et al. 2006, A\&A, 451,651

Jorissen, A., Van Eck, S., Van Winckel, H., et al. 2016, A\&A, 586, A158

Karakas, A. I., \& Lattanzio, J. C. 2014, PASA, 31, e030

Kipper, T., \& Jorgensen, U. G. 1994, A\&A, 290, 148

Lee, Y. S., Suda, T., Beers, T. C., \& Stancliffe, R. J. 2014, ApJ, 788,131

Lee, Y. S., Beers, T. C., Masseron, T., et al. 2013, AJ, 146, 132

Li, H., Aoki, W., Zhao, G., et al. 2015, PASJ, 67, 84

Liu, S., Nissen, P. E., Schuster, W. J., et al. 2012, A\&A, 541, A48

Lucatello, S. 2004, PhD thesis, Dipartimento di Astronomia, Università di Padova

Lucatello, S., Gratton, R. G., Beers, T. C., \& Carretta, E. 2005a, ApJ, 625, 833

Lucatello, S., Tsangarides, S., Beers, T. C., et al. 2005b, ApJ, 625,825

Lugaro, M., Campbell, S. W., \& de Mink, S. E. 2009, PASA, 26, 322

Maeder, A., \& Meynet, G. 2015, A\&A, 580, A32

Maeder, A., Meynet, G., \& Chiappini, C. 2015, A\&A, 576, A56

Masseron, T., Johnson, J. A., Lucatello, S., et al. 2012, ApJ, 751, 14

Masseron, T., Johnson, J. A., Plez, B., et al. 2010, A\&A, 509, A93+

Matrozis, E., \& Stancliffe, R. J. 2016, A\&A, 592, A29

McClure, R. D., \& Woodsworth, A. W. 1990, ApJ, 352, 709

Meynet, G., Ekström, S., \& Maeder, A. 2006, A\&A, 447, 623

Meynet, G., Hirschi, R., Ekstrom, S., et al. 2010, A\&A, 521, A30

Nomoto, K., Kobayashi, C., \& Tominaga, N. 2013, ARA\&A, 51, 457

Norris, J. E., Christlieb, N., Korn, A. J., et al. 2007, ApJ, 670, 774

Norris, J. E., Bessell, M. S., Yong, D., et al. 2013, ApJ, 762, 25

Pedregosa, F., Varoquaux, G., Gramfort, A., et al. 2011, Journal of Machine Learning Research, 12, 2825

Pereira, C. B., \& Drake, N. A. 2009, A\&A, 496, 791

Pereira, C. B., Jilinski, E., Drake, N. A., et al. 2012, A\&A, 543, A58

Placco, V. M., Frebel, A., Beers, T. C., et al. 2014a, ApJ, 781, 40 -. 2013, ApJ, 770, 104
Placco, V. M., Frebel, A., Beers, T. C., \& Stancliffe, R. J. 2014b, ApJ, 797, 21

Placco, V. M., Beers, T. C., Roederer, I. U., et al. 2014c, ApJ, 790, 34

Placco, V. M., Beers, T. C., Ivans, I. I., et al. 2015, ApJ, 812, 109

Placco, V. M., Frebel, A., Beers, T. C., et al. 2016, ArXiv e-prints, arXiv:1609.02134

Plez, B., \& Cohen, J. G. 2005, A\&A, 434, 1117

Preston, G. W., \& Sneden, C. 2001, AJ, 122, 1545

Roederer, I. U., Placco, V. M., \& Beers, T. C. 2016, ApJL, 824, L19

Roederer, I. U., Preston, G. W., Thompson, I. B., Shectman, S. A., \& Sneden, C. 2014a, ApJ, 784, 158

Roederer, I. U., Preston, G. W., Thompson, I. B., et al. 2014b, AJ, 147, 136

Roederer, I. U., Frebel, A., Shetrone, M. D., et al. 2008, ApJ, 679,1549

Ryan, S. G., Aoki, W., Norris, J. E., \& Beers, T. C. 2005, ApJ, 635,349

Schuler, S. C., Cunha, K., Smith, V. V., et al. 2007, ApJL, 667, L81

Simmerer, J., Sneden, C., Cowan, J. J., et al. 2004, ApJ, 617, 1091

Sivarani, T., Beers, T. C., Bonifacio, P., et al. 2006, A\&A, 459, 125

Sneden, C., Cowan, J. J., Lawler, J. E., et al. 2003, ApJ, 591, 936

Spite, M., Caffau, E., Bonifacio, P., et al. 2013, A\&A, 552, A107

Stancliffe, R. J. 2010, MNRAS, 403, 505

Stancliffe, R. J., \& Glebbeek, E. 2008, MNRAS, 389, 1828

Stancliffe, R. J., Glebbeek, E., Izzard, R. G., \& Pols, O. R. 2007, A\&A, 464, L57

Starkenburg, E., Shetrone, M. D., McConnachie, A. W., \& Venn, K. A. 2014, MNRAS, 441, 1217

Suda, T., Aikawa, M., Machida, M. N., Fujimoto, M. Y., \& Iben, Jr., I. 2004, ApJ, 611, 476

Suda, T., Yamada, S., Katsuta, Y., et al. 2011, MNRAS, 412, 843

Suda, T., Katsuta, Y., Yamada, S., et al. 2008, PASJ, 60, 1159

Suda, T., Komiya, Y., Yamada, S., et al. 2013, MNRAS, 432, L46

Susa, H., Hasegawa, K., \& Tominaga, N. 2014, ApJ, 792, 32

Thompson, I. B., Ivans, I. I., Bisterzo, S., et al. 2008, ApJ, 677, 556

Tominaga, N., Iwamoto, N., \& Nomoto, K. 2014, ApJ, 785, 98

Tominaga, N., Umeda, H., \& Nomoto, K. 2007, ApJ, 660, 516

Tumlinson, J. 2007a, ApJ, 665, 1361

-. 2007b, ApJL, 664, L63

Umeda, H., \& Nomoto, K. 2003, Nature, 422, 871

—. 2005, ApJ, 619, 427

Vanture, A. D. 1992, AJ, 103, 2035

Yamada, S., Suda, T., Komiya, Y., Aoki, W., \& Fujimoto, M. Y. 2013, MNRAS, 436, 1362

Yong, D., Norris, J. E., Bessell, M. S., et al. 2013, ApJ, 762, 26

York, D. G., Adelman, J., Anderson, Jr., J. E., et al. 2000, AJ, 120,1579 
Table 1. Literature Data and Classifications for Carbon-Enhanced Metal-Poor (CEMP) Stars

\begin{tabular}{|c|c|c|c|c|c|c|c|c|c|c|c|c|}
\hline $\begin{array}{c}\text { Name } \\
(1) \\
\end{array}$ & $\begin{array}{l}\mathrm{T}_{\text {eff }} \\
(\mathrm{K}) \\
(2) \\
\end{array}$ & $\begin{array}{c}\log g \\
(\mathrm{cgs}) \\
(3) \\
\end{array}$ & {$[\mathrm{Fe} / \mathrm{H}]$} & {$[\mathrm{C} / \mathrm{Fe}]$} & {$[\mathrm{C} / \mathrm{Fe}]_{c}$} & $\begin{array}{c}A(\mathrm{C}) \\
\text { (corrected) } \\
(7) \\
\end{array}$ & {$[\mathrm{Ba} / \mathrm{Fe}]$} & {$[\mathrm{Eu} / \mathrm{Fe}]$} & $\begin{array}{c}\text { Class } \\
(\mathrm{Ba} \mid A(\mathrm{C})) \\
(10)\end{array}$ & Binarity ${ }^{\mathrm{a}}$ & $\begin{array}{c}\text { Interesting } \\
\text { outliers } \\
(12) \\
\end{array}$ & $\begin{array}{c}\text { Reference } \\
\text { (13) } \\
\end{array}$ \\
\hline \multicolumn{13}{|c|}{ Group I Stars } \\
\hline 52972-1213-507 & 6463 & 4.34 & -2.98 & 2.81 & 2.81 & 8.26 & 1.7 & $\cdots$ & $(\mathrm{s} \mid \mathrm{s})$ & $\cdots$ & & Yong et al. (2013) \\
\hline $\mathrm{BD}+042466$ & 5100 & 1.8 & -1.9 & 1.24 & 1.34 & 7.87 & 1.63 & $\ldots$ & $(\mathrm{s} \mid \mathrm{s})$ & $\ldots$ & & Pereira \& Drake (2009) \\
\hline BS $16077-077$ & 5900 & 3.19 & -2.05 & 2.39 & 2.42 & 8.80 & 0.75 & 0.04 & $\left(\begin{array}{lll}s & s\end{array}\right)$ & $\cdots$ & & Allen et al. (2012) \\
\hline BS $16080-175$ & 6240 & 3.7 & -1.86 & 1.94 & 1.94 & 8.51 & 1.40 & -0.44 & $\left(\begin{array}{lll}s & s\end{array}\right)$ & $\cdots$ & & Allen et al. (2012) \\
\hline BS $17436-058$ & 5430 & 2.2 & -1.9 & 1.71 & 1.73 & 8.26 & 1.68 & 1.28 & $(\mathrm{r} / \mathrm{s} \mid \mathrm{r} / \mathrm{s})$ & $\cdots$ & & Allen et al. (2012) \\
\hline CD-62 1346 & 5300 & 1.70 & -1.57 & 0.93 & 1.04 & 7.90 & 1.51 & $\cdots$ & $(\mathrm{s} \mid \mathrm{s})$ & $\cdots$ & & Pereira et al. (2012) \\
\hline CS 22879-029 & 5920 & 3.7 & -2.55 & 1.46 & 1.46 & 7.34 & 1.39 & 0.59 & $\left(\begin{array}{lll}s & s\end{array}\right)$ & $\cdots$ & & Roederer et al. (2014b) \\
\hline CS 22880-074 & 5621 & 3.5 & -2.29 & 1.30 & 1.30 & 7.44 & 1.31 & 0.54 & $(\mathrm{~s} \mid \mathrm{s})$ & $\ldots$ & & Yong et al. (2013) \\
\hline CS 22881-036 & 5940 & 3.7 & -2.34 & 1.96 & 1.96 & 8.05 & 2.00 & 0.55 & $\left(\begin{array}{lll}\mathrm{s} \mid & \mathrm{s}\end{array}\right)$ & $\cdots$ & & Roederer et al. (2014b) \\
\hline CS 22887-048 & 6500 & 3.35 & -1.7 & 1.93 & 1.95 & 8.68 & 1.95 & 1.48 & $(\mathrm{r} / \mathrm{s} \mid \mathrm{r} / \mathrm{s})$ & $\cdots$ & & Allen et al. (2012) \\
\hline CS 22891-171 & 5100 & 1.6 & -2.20 & 1.66 & 1.78 & 8.01 & 2.52 & $\cdots$ & $(\mathrm{s} \mid \mathrm{s})$ & $\cdots$ & & Allen et al. (2012) \\
\hline CS 22896-136 & 6190 & 3.85 & -2.41 & 1.30 & 1.30 & 7.32 & 1.44 & 0.81 & $(\mathrm{~s} \mid \mathrm{s})$ & $\cdots$ & & Roederer et al. (2014b) \\
\hline CS 22898-027 & 6250 & 3.7 & -2.21 & 2.21 & 2.21 & 8.43 & 2.27 & 2.01 & $(\mathrm{r} / \mathrm{s} \mid \mathrm{r} / \mathrm{s})$ & $\cdots$ & & Masseron et al. (2012) \\
\hline CS 22942-019 & 5000 & 2.4 & -2.64 & 2.00 & 2.02 & 7.81 & 1.92 & 0.79 & $(\mathrm{~s} \mid \mathrm{s})$ & $2^{\mathrm{b}}$ & & Aoki et al. (2002b) \\
\hline CS 22943-201 & 5970 & 2.45 & -2.68 & 1.88 & 1.90 & 7.65 & -0.54 & $<0.73$ & $($ no $\mid s)$ & $\cdots$ & $\sqrt{ }$ & Roederer et al. (2014b) \\
\hline CS 22945-024 & 5120 & 2.35 & -2.58 & 2.30 & 2.32 & 8.17 & 1.43 & 0.44 & $\left(\begin{array}{lll}s & s\end{array}\right)$ & $\cdots$ & & Roederer et al. (2014b) \\
\hline CS $22947-187$ & 5300 & 1.4 & -2.58 & 1.25 & 1.51 & 7.36 & 1.53 & 0.55 & $(\mathrm{~s} \mid \mathrm{s})$ & $\cdots$ & & Roederer et al. (2014b) \\
\hline CS 22949-008A & 6300 & 3.5 & -2.09 & 1.51 & 1.51 & 7.85 & 1.31 & $<0.57$ & $(\mathrm{~s} \mid \mathrm{s})$ & $\cdots$ & & Masseron et al. (2012) \\
\hline CS 22949-008B & 5300 & 4.7 & -2.09 & 1.51 & 1.51 & 7.85 & 1.31 & $<1.37$ & $\left(\begin{array}{lll}s \mid & s\end{array}\right)$ & $\cdots$ & & Masseron et al. (2012) \\
\hline CS 22956-028 & 6900 & 3.9 & -2.08 & 1.73 & 1.73 & 8.08 & 0.37 & $\cdots$ & $(\mathrm{s} \mid \mathrm{s})$ & $\cdots$ & & Sneden et al. (2003) \\
\hline CS 22956-102 & 6220 & 3.85 & -2.39 & 2.21 & 2.21 & 8.25 & 1.99 & 1.00 & $\left(\begin{array}{lll}s & s\end{array}\right)$ & $\cdots$ & & Roederer et al. (2014b) \\
\hline CS 22957-027 & 5220 & 2.65 & -3.19 & 2.61 & 2.63 & 7.87 & -0.81 & $<1.07$ & $($ no $\mid \mathrm{s})$ & 2 & $\checkmark$ & Roederer et al. (2014b) \\
\hline CS 22958-042 & 5760 & 3.55 & -3.40 & 2.56 & 2.56 & 7.59 & $<-0.61$ & $<1.54$ & $($ no $\mid$ s) & $\cdots$ & $\sqrt{ }$ & Roederer et al. (2014b) \\
\hline CS $22964-161 \mathrm{~A}$ & 6050 & 3.7 & -2.37 & 1.58 & 1.58 & 7.64 & 1.36 & $\ldots$ & $(\mathrm{s} \mid \mathrm{s})$ & $2^{\mathrm{c}}$ & & Thompson et al. (2008) \\
\hline CS 22964-161B & 5850 & 4.1 & -2.39 & 1.40 & 1.40 & 7.44 & 1.30 & $\cdots$ & $\left(\begin{array}{lll}s & s\end{array}\right)$ & $2^{\mathrm{c}}$ & $\sqrt{ }$ & Thompson et al. (2008) \\
\hline CS 22967-07 & 6479 & 4.2 & -1.77 & 1.89 & 1.90 & 8.56 & 2.03 & 0.80 & $(\mathrm{~s} \mid \mathrm{s})$ & $\cdots$ & & Lucatello (2004) \\
\hline CS 29495-42 & 5544 & 3.4 & -1.84 & 1.43 & 1.43 & 8.02 & 1.77 & 0.80 & $\left(\begin{array}{lll}s & s\end{array}\right)$ & $\cdots$ & & Lucatello (2004) \\
\hline CS 29497-030 & 7000 & 4.0 & -2.52 & 2.37 & 2.37 & 8.28 & 2.75 & 1.71 & $\left(\begin{array}{lll}s & s\end{array}\right)$ & $\cdots$ & & Roederer et al. (2014b) \\
\hline CS 29497-034 & 4983 & 1.96 & -3.0 & 2.72 & 2.76 & 8.19 & 2.28 & 1.79 & $(\mathrm{r} / \mathrm{s} \mid \mathrm{r} / \mathrm{s})$ & 2 & & Yong et al. (2013) \\
\hline CS 29498-043 & 4440 & 0.50 & -3.87 & 2.75 & 3.06 & 7.62 & -0.49 & $<0.23$ & $($ no $\mid$ s) & 1 & $\sqrt{ }$ & Roederer et al. (2014b) \\
\hline CS 29509-027 & 6850 & 3.5 & -2.42 & 1.74 & 1.74 & 7.75 & 1.50 & $<1.12$ & $(\mathrm{~s} \mid \mathrm{s})$ & $\cdots$ & & Roederer et al. (2014b) \\
\hline CS 29512-073 & 5650 & 3.6 & -2.10 & 1.24 & 1.24 & 7.57 & 1.60 & 0.53 & $(\mathrm{~s} \mid \mathrm{s})$ & $\cdots$ & & Roederer et al. (2014b) \\
\hline CS 29513-014 & 5440 & 1.55 & -2.32 & 1.03 & 1.27 & 7.38 & 1.38 & 0.24 & $(\mathrm{~s} \mid \mathrm{s})$ & $\cdots$ & & Roederer et al. (2014b) \\
\hline CS 29526-110 & 6800 & 4.1 & -2.11 & 2.08 & 2.08 & 8.40 & 3.03 & $\cdots$ & $(\mathrm{r} / \mathrm{s} \mid \mathrm{r} / \mathrm{s})$ & $\cdots$ & & Aoki et al. (2008) \\
\hline CS 29528-028 & 6800 & 4.0 & -2.81 & 2.78 & 2.78 & 8.40 & 3.31 & $\cdots$ & $(\mathrm{s} \mid \mathrm{s})$ & $\cdots$ & & Aoki et al. (2007) \\
\hline CS 30301-015 & 4889 & 1.73 & -2.73 & 1.60 & 1.74 & 7.44 & 1.45 & 0.22 & $\left(\begin{array}{lll}\mathrm{s} & \mathrm{s}\end{array}\right)$ & 1 & $\sqrt{ }$ & Yong et al. (2013) \\
\hline CS 30315-91 & 5536 & 3.4 & -1.64 & 1.4 & 1.41 & 8.20 & 1.56 & 0.40 & $\left(\begin{array}{lll}s & s\end{array}\right)$ & $\cdots$ & & Lucatello (2004) \\
\hline CS $30323-107$ & 6126 & 4.4 & -1.71 & 1.23 & 1.23 & 7.95 & 1.84 & 0.60 & $(\mathrm{~s} \mid \mathrm{s})$ & $\cdots$ & & Lucatello (2004) \\
\hline CS 30338-089 & 4886 & 1.72 & -2.78 & 2.06 & 2.17 & 7.82 & 2.30 & $\cdots$ & $\left(\begin{array}{lll}s \mid & s\end{array}\right)$ & $\cdots$ & & Yong et al. (2013) \\
\hline CS 31062-012 & 6190 & 4.47 & -2.67 & 2.12 & 2.12 & 7.88 & 2.32 & $\cdots$ & $(\mathrm{s} \mid \mathrm{s})$ & $\cdots$ & & Yong et al. (2013) \\
\hline CS 31062-050 & 5607 & 3.49 & -2.28 & 2.00 & 2.00 & 8.15 & 2.30 & 1.90 & $(\mathrm{r} / \mathrm{s} \mid \mathrm{r} / \mathrm{s})$ & $\cdots$ & & Yong et al. (2013) \\
\hline G $24-25$ & 5828 & 3.86 & -1.40 & 1.00 & 1.00 & 8.03 & 1.34 & 0.61 & $\left(\begin{array}{lll}\mathrm{s} \mid & \mathrm{s}\end{array}\right)$ & $\cdots$ & & Liu et al. (2012) \\
\hline HD 005223 & 4500 & 1.0 & -2.11 & 1.58 & 1.7 & 8.02 & 1.88 & $\cdots$ & $(\mathrm{s} \mid \mathrm{s})$ & $2^{\mathrm{d}}$ & & Goswami et al. (2006) \\
\hline HD 187216 & 3500 & 0.4 & -2.48 & 1.38 & 1.64 & 7.59 & 2.30 & $\cdots$ & $\left(\begin{array}{lll}s & s\end{array}\right)$ & $\cdots$ & & Kipper \& Jorgensen (1994) \\
\hline HD 187861 & 5320 & 2.4 & -2.30 & 2.67 & 2.69 & 8.82 & $\cdots$ & $\cdots$ & $\left(\begin{array}{lll}\mathrm{s} & \mathrm{s}\end{array}\right)$ & $\cdots$ & & Vanture (1992) \\
\hline HD 196944 & 5170 & 1.60 & -2.41 & 1.08 & 1.31 & 7.33 & 1.23 & -0.11 & $(\mathrm{~s} \mid \mathrm{s})$ & $2^{\mathrm{e}}$ & & Placco et al. (2015) \\
\hline HD 198269 & 4500 & 1.5 & -2.08 & 2.55 & 2.59 & 8.94 & 1.26 & $\cdots$ & $\left(\begin{array}{lll}\mathrm{s} & \mathrm{s}\end{array}\right)$ & $2^{\mathrm{d}}$ & & Goswami et al. (2016) \\
\hline HD 201626 & 5175 & 2.80 & -1.51 & 1.48 & 1.50 & 8.42 & 1.73 & 0.95 & $(\mathrm{~s} \mid \mathrm{s})$ & $2^{\mathrm{d}}$ & & Placco et al. (2015) \\
\hline HD 209621 & 4500 & 2.0 & -1.99 & 1.26 & 1.31 & 7.75 & 1.76 & 1.42 & $(r / s \mid r / s)$ & $2^{\mathrm{d}}$ & & Goswami \& Aoki (2010) \\
\hline HD 224959 & 5050 & 2.1 & -2.47 & 2.04 & 2.08 & 8.04 & 2.11 & 2.04 & $(\mathrm{r} / \mathrm{s} \mid \mathrm{r} / \mathrm{s})$ & $2^{\mathrm{d}}$ & & Goswami et al. (2016) \\
\hline HE $0002-1037^{f}$ & 4673 & 1.28 & -3.75 & 3.19 & 3.43 & 8.11 & 1.67 & $\cdots$ & $(\mathrm{s} \mid \mathrm{s})$ & 2 & & Hansen et al. (2016c) \\
\hline HE $0007-1832$ & 6500 & 3.8 & -2.84 & 2.87 & 2.87 & 8.46 & 0.09 & $<1.75$ & $($ no $\mid$ s) & $\cdots$ & $\checkmark$ & Cohen et al. (2013) \\
\hline HE $0012-1441$ & 5750 & 3.5 & -2.57 & 1.80 & 1.80 & 7.66 & 1.15 & $\cdots$ & $(\mathrm{s} \mid \mathrm{s})$ & $\cdots$ & & Cohen et al. (2013) \\
\hline HE $0017+0055$ & 4146 & 0.41 & -2.8 & 1.99 & 2.20 & 7.83 & $<1.62$ & $\cdots$ & $(\mathrm{s} \mid \mathrm{s})$ & 2 & & Hansen et al. (2016c) \\
\hline HE $0017-4346$ & 6198 & 3.8 & -3.12 & 3.11 & 3.11 & 8.42 & 1.28 & $<1.04$ & $\left(\begin{array}{lll}\mathrm{s} & \mathrm{s}\end{array}\right)$ & $\cdots$ & & Cohen et al. (2013) \\
\hline HE 0024-2523 & 6625 & 4.3 & -2.65 & 2.63 & 2.63 & 8.41 & 1.56 & $\cdots$ & $\left(\begin{array}{lll}s & s\end{array}\right)$ & $\cdots$ & & Masseron et al. (2012) \\
\hline HE $0054-2542$ & 5300 & 2.7 & -2.48 & 2.13 & 2.15 & 8.10 & 1.52 & 0.78 & $(\mathrm{~s} \mid \mathrm{s})$ & $\cdots$ & & Hansen et al. (2015a) \\
\hline HE 0058-0244 & 5620 & 3.4 & -2.81 & 2.14 & 2.14 & 7.76 & 1.97 & 1.58 & $(r / s \mid r / s)$ & $\cdots$ & & Cohen et al. (2013) \\
\hline HE 0058-3449 & 5400 & 3.0 & -2.1 & 1.0 & 1.0 & 7.3 & 1.8 & $\cdots$ & $\left(\begin{array}{lll}s \mid & s\end{array}\right)$ & $\cdots$ & & Hansen et al. (2016a) \\
\hline
\end{tabular}


YOON ET AL.

Table 1 (continued)

\begin{tabular}{|c|c|c|c|c|c|c|c|c|c|c|c|c|}
\hline (1) & $\begin{array}{l}\mathrm{T}_{\text {eff }} \\
(\mathrm{K}) \\
(2) \\
\end{array}$ & $\begin{array}{c}\log g \\
(\mathrm{cgs}) \\
(3)\end{array}$ & {$[\mathrm{Fe} / \mathrm{H}]$} & {$[\mathrm{C} / \mathrm{Fe}]$} & {$[\mathrm{C} / \mathrm{Fe}]_{c}$} & $\begin{array}{c}A(\mathrm{C}) \\
\text { (corrected) } \\
(7)\end{array}$ & {$[\mathrm{Ba} / \mathrm{Fe}]$} & {$[\mathrm{Eu} / \mathrm{Fe}]$} & $\begin{array}{c}\text { Class } \\
(\mathrm{Ba} \mid A(\mathrm{C})) \\
(10)\end{array}$ & Binarity $^{a}$ & $\begin{array}{c}\text { Interesting } \\
\text { outliers } \\
(12) \\
\end{array}$ & Reference \\
\hline HE $0100-1622$ & 5400 & 3.0 & -2.93 & 2.75 & 2.77 & 8.27 & $<-1.80$ & $<0.80$ & $(\mathrm{no} \mid \mathrm{s})$ & $\cdots$ & $\checkmark$ & Hansen et al. (2015a) \\
\hline HE 0111-1346 & 4609 & 0.58 & -1.91 & 1.70 & 1.79 & 8.31 & $<2.32$ & $\cdots$ & $(\mathrm{s} \mid \mathrm{s})$ & 2 & & Hansen et al. (2016c) \\
\hline HE 0131-3953 & 5928 & 3.83 & -2.68 & 2.44 & 2.44 & 8.16 & 2.16 & 1.61 & $(\mathrm{~s} \mid \mathrm{s})$ & $\cdots$ & & Barklem et al. (2005) \\
\hline HE $0134-2504$ & 5204 & 2.5 & -2.79 & 2.02 & 2.02 & 7.66 & 1.65 & $\cdots$ & $(\mathrm{s} \mid \mathrm{s})$ & $\cdots$ & & Cohen et al. (2013) \\
\hline HE $0143-0441$ & 6276 & 3.84 & -2.32 & 1.82 & 1.82 & 7.93 & 2.42 & 1.69 & $(s \mid s)$ & $\cdots$ & & Yong et al. (2013) \\
\hline HE 0151-0341 & 4771 & 1.34 & -2.46 & 2.10 & 2.20 & 8.17 & 1.22 & $\cdots$ & $(\mathrm{s} \mid \mathrm{s})$ & 2 & & Hansen et al. (2016c) \\
\hline HE 0206-1916 & 5073 & 2.23 & -2.52 & 2.10 & 2.12 & 8.03 & 1.99 & $\cdots$ & $(\mathrm{s} \mid \mathrm{s})$ & 1 & $\sqrt{ }$ & Yong et al. (2013) \\
\hline HE 0207-1423 & 5023 & 2.07 & -2.95 & 2.38 & 2.41 & 7.89 & 1.73 & $\cdots$ & $(\mathrm{s} \mid \mathrm{s})$ & $\cdots$ & & Yong et al. (2013) \\
\hline HE $0212-0557$ & 5075 & 2.2 & -2.32 & 1.95 & 1.97 & 8.08 & 2.18 & $\cdots$ & $(\mathrm{s} \mid \mathrm{s})$ & $\cdots$ & & Cohen et al. (2013) \\
\hline HE 0219-1739 & 4238 & 0.47 & -3.09 & 1.90 & 2.21 & 7.55 & $<-1.39$ & $\cdots$ & $($ no $\mid \mathrm{s})$ & 2 & $\sqrt{ }$ & Hansen et al. (2016b) \\
\hline HE $0231-4016$ & 5972 & 3.59 & -2.08 & 1.32 & 1.32 & 7.67 & 1.42 & $\cdots$ & $(\mathrm{s} \mid \mathrm{s})$ & $\cdots$ & & Barklem et al. (2005) \\
\hline HE $0241-3512$ & 4600 & 1.2 & -1.8 & 1.3 & 1.4 & 8.1 & 2.0 & $\cdots$ & $(s \mid s)$ & $\cdots$ & & Hansen et al. (2016a) \\
\hline HE $0243-3044$ & 5400 & 3.2 & -2.58 & 2.43 & 2.45 & 8.30 & 1.96 & 1.90 & $(\mathrm{r} / \mathrm{s} \mid \mathrm{r} / \mathrm{s})$ & $\cdots$ & & Hansen et al. (2015a) \\
\hline HE 0251-3216 & 5750 & 3.7 & -3.20 & 2.53 & 2.53 & 7.76 & 1.28 & $\cdots$ & $(\mathrm{s} \mid \mathrm{s})$ & $\cdots$ & & Cohen et al. (2013) \\
\hline HE 0319-0215 & 4448 & 0.62 & -2.30 & 2.00 & 2.08 & 8.21 & 0.52 & $\cdots$ & $(\mathrm{s} \mid \mathrm{s})$ & 2 & & Hansen et al. (2016c) \\
\hline HE $0322-1504$ & 4460 & 0.8 & -1.95 & 2.31 & 2.34 & 8.83 & 2.84 & $\cdots$ & $(\mathrm{s} \mid \mathrm{s})$ & $\cdots$ & & Beers et al. (2007) \\
\hline HE $0336+0113$ & 5819 & 3.59 & -2.60 & 2.25 & 2.25 & 8.08 & 2.69 & 1.22 & $(s \mid s)$ & $\cdots$ & & Yong et al. (2013) \\
\hline HE $0338-3945$ & 6160 & 4.13 & -2.43 & 2.08 & 2.08 & 8.08 & 2.35 & 1.93 & $(\mathrm{r} / \mathrm{s} \mid \mathrm{r} / \mathrm{s})$ & $\cdots$ & & Jonsell et al. (2006) \\
\hline HE $0400-2030$ & 5600 & 3.5 & -1.78 & 1.15 & 1.15 & 7.80 & 1.67 & $\cdots$ & $(\mathrm{s} \mid \mathrm{s})$ & $\cdots$ & & Aoki et al. (2007) \\
\hline HE 0405-0526 & 5083 & 3.86 & -2.18 & 0.92 & 0.92 & 7.17 & -0.22 & $\cdots$ & $($ no $\mid$ s) & 1 & $\sqrt{ }$ & Hansen et al. (2016b) \\
\hline HE 0414-0343 & 4863 & 1.25 & -2.24 & 1.44 & 1.60 & 7.79 & 1.87 & 1.23 & $(\mathrm{r} / \mathrm{s} \mid \mathrm{r} / \mathrm{s})$ & 2 & & Hollek et al. (2015) \\
\hline HE 0430-1609 & 4621 & 0.98 & -3.00 & 1.14 & 1.66 & 7.09 & 1.62 & $\cdots$ & $(\mathrm{s} \mid \mathrm{no})$ & 2 & $\checkmark$ & Hansen et al. (2016c) \\
\hline HE $0430-4404$ & 6214 & 4.27 & -2.07 & 1.39 & 1.39 & 7.75 & 1.57 & $\cdots$ & $(\mathrm{s} \mid \mathrm{s})$ & $\cdots$ & & Barklem et al. (2005) \\
\hline HE 0441-0652 & 4811 & 1.52 & -2.77 & 1.38 & 1.63 & 7.29 & 1.20 & $\cdots$ & $(\mathrm{s} \mid \mathrm{s})$ & 2 & $\sqrt{ }$ & Yong et al. (2013) \\
\hline HE $0450-4902$ & 6300 & 4.5 & -3.07 & 2.03 & 2.03 & 7.39 & 1.21 & $<2.00$ & $\left(\begin{array}{ll}s \mid & s\end{array}\right)$ & $\cdots$ & & Hansen et al. (2015a) \\
\hline HE 0507-1653 & 5000 & 2.4 & -1.43 & 1.30 & 1.32 & 8.32 & 1.93 & & $(\mathrm{~s} \mid \mathrm{s})$ & 2 & & Aoki et al. (2007) \\
\hline HE $0534-4548$ & 4250 & 1.5 & -1.75 & 1.51 & 1.59 & 8.27 & 0.64 & $\cdots$ & $(\mathrm{s} \mid \mathrm{s})$ & $\cdots$ & & Beers et al. (2007) \\
\hline HE $0854+0151$ & 5015 & 0.37 & -1.80 & 1.60 & 1.70 & 8.33 & 0.82 & $\cdots$ & $\left(\begin{array}{ll}s \mid & s\end{array}\right)$ & 2 & & Hansen et al. (2016c) \\
\hline HE $0959-1424$ & 5790 & 0.64 & -1.42 & 2.30 & 2.33 & 9.34 & 1.24 & $\cdots$ & $(\mathrm{s} \mid \mathrm{s})$ & 2 & & Hansen et al. (2016c) \\
\hline HE 1005-1439 & 5202 & 2.55 & -3.09 & 2.48 & 2.5 & 7.84 & 1.17 & $\cdots$ & $\left(\begin{array}{lll}s \mid & s\end{array}\right)$ & $\cdots$ & & Yong et al. (2013) \\
\hline HE 1031-0020 & 5043 & 2.13 & -2.79 & 1.63 & 1.65 & 7.29 & 1.61 & $<0.91$ & $\left(\begin{array}{ll}s \mid & s\end{array}\right)$ & 2 & $\checkmark$ & Yong et al. (2013) \\
\hline HE $1045+0226$ & 5077 & 2.2 & -2.25 & 1.18 & 1.20 & 7.38 & 1.24 & 0.27 & $(\mathrm{~s} \mid \mathrm{s})$ & 1 & $\sqrt{ }$ & Cohen et al. (2013) \\
\hline HE $1046-1352$ & 5557 & 2.09 & -2.76 & 3.30 & 3.33 & 9.00 & 1.38 & $\cdots$ & $(\mathrm{s} \mid \mathrm{s})$ & 2 & & Hansen et al. (2016c) \\
\hline HE $1105+0027^{g}$ & 6132 & 3.45 & -2.42 & 1.96 & 1.96 & 7.97 & 2.40 & 1.80 & $(\mathrm{~s} \mid \mathrm{s})$ & 1 & $\sqrt{ }$ & Barklem et al. (2005) \\
\hline HE 1111-3026 & 5000 & 2.0 & -2.05 & 1.18 & 1.24 & 7.62 & 1.31 & $\cdots$ & $\left(\begin{array}{lll}s & s\end{array}\right)$ & $\cdots$ & & Cohen et al. (2013) \\
\hline HE 1133-0555 & 5526 & 1.31 & -2.40 & 2.20 & 2.28 & 8.31 & -0.58 & $\cdots$ & $($ no $\mid \mathrm{s})$ & 1 & $\sqrt{ }$ & Hansen et al. (2016b) \\
\hline HE $1135+0139$ & 5487 & 1.8 & -2.33 & 1.18 & 1.33 & 7.43 & 1.08 & 0.33 & $(\mathrm{~s} \mid \mathrm{s})$ & $\cdots$ & & Barklem et al. (2005) \\
\hline HE $1150-0428$ & 5208 & 2.54 & -3.47 & 2.37 & 2.39 & 7.35 & -0.48 & $<1.49$ & $($ no $\mid \mathrm{s})$ & 2 & $\sqrt{ }$ & Yong et al. (2013) \\
\hline HE 1152-0355 & 4000 & 1.0 & -1.32 & 0.59 & 0.73 & 7.84 & 1.59 & $\cdots$ & $(\mathrm{s} \mid \mathrm{s})$ & $\cdots$ & & Goswami et al. (2006) \\
\hline HE $1157-0518$ & 4900 & 2.0 & -2.39 & 2.16 & 2.21 & 8.25 & 2.18 & $\cdots$ & $(\mathrm{s} \mid \mathrm{s})$ & $\cdots$ & & Aoki et al. (2007) \\
\hline HE $1159-0525$ & 4838 & 1.5 & -2.96 & 2.03 & 2.22 & 7.69 & 1.53 & 0.74 & $(\mathrm{~s} \mid \mathrm{s})$ & $\cdots$ & & Cohen et al. (2013) \\
\hline HE $1302-0954$ & 5120 & 2.4 & -2.25 & 1.17 & 1.19 & 7.37 & $<-0.53$ & $\cdots$ & $($ no $\mid$ s) & 1 & $\sqrt{ }$ & Hansen et al. (2016b) \\
\hline HE $1305+0007$ & 4750 & 2.0 & -2.08 & 1.85 & 1.90 & 8.25 & 2.40 & $\cdots$ & $(\mathrm{s} \mid \mathrm{s})$ & $\cdots$ & & Goswami et al. (2006) \\
\hline HE $1305+0132$ & 4462 & 0.8 & -2.45 & 2.59 & 2.65 & 8.63 & $\cdots$ & $\cdots$ & $(s \mid s)$ & $\cdots$ & & Schuler et al. (2007) \\
\hline HE $1315-2035$ & 5100 & 2.2 & -2.5 & 2.5 & 2.5 & 8.5 & $<2.6$ & $\cdots$ & $(s \mid s)$ & $\cdots$ & & Hansen et al. (2016a) \\
\hline HE 1319-1935 & 4691 & 1.27 & -2.22 & 1.45 & 1.60 & 7.81 & 1.68 & $\cdots$ & $\left(\begin{array}{lll}s & s\end{array}\right)$ & $\cdots$ & & Yong et al. (2013) \\
\hline HE $1405-0822$ & 5220 & 1.7 & -2.37 & 1.90 & 1.98 & 8.04 & 1.92 & 1.52 & $(\mathrm{~s} \mid \mathrm{s})$ & $\cdots$ & & Cui et al. (2013) \\
\hline HE $1410-0004$ & 4985 & 2.0 & -3.04 & 2.10 & 2.14 & 7.53 & 1.17 & $<2.44$ & $\left(\begin{array}{lll}s & s\end{array}\right)$ & $\cdots$ & & Masseron et al. (2012) \\
\hline HE $1410+0213$ & 5000 & 2.0 & -2.14 & 1.92 & 1.97 & 8.21 & -0.26 & $\cdots$ & $($ no $\mid$ s) & 1 & $\sqrt{ }$ & Cohen et al. (2013) \\
\hline HE $1429-0551$ & 4757 & 1.39 & -2.60 & 2.28 & 2.38 & 8.21 & 1.47 & $\cdots$ & $(\mathrm{s} \mid \mathrm{s})$ & $2 ?^{\mathrm{h}}$ & & Yong et al. (2013) \\
\hline HE $1430-0919$ & 4900 & 1.6 & -2.5 & 2.2 & 2.3 & 8.2 & 1.5 & $\cdots$ & $(s \mid s)$ & $\cdots$ & & Hansen et al. (2016a) \\
\hline HE $1430-1123$ & 5915 & 3.75 & -2.70 & 1.81 & 1.81 & 7.53 & 1.77 & $\cdots$ & $(\mathrm{s} \mid \mathrm{s})$ & $\cdots$ & & Barklem et al. (2005) \\
\hline HE 1431-0245 & 5200 & 2.3 & -2.5 & 2.4 & 2.42 & 8.4 & 1.9 & $\cdots$ & $\left(\begin{array}{ll}s \mid & s\end{array}\right)$ & $\cdots$ & & Hansen et al. (2016a) \\
\hline HE $1434-1442$ & 5420 & 3.2 & -2.43 & 2.16 & 2.17 & 8.17 & 1.23 & $\cdots$ & $(\mathrm{s} \mid \mathrm{s})$ & $\cdots$ & & Cohen et al. (2013) \\
\hline HE $1439-1420$ & 6056 & 3.8 & 3.02 & 2.05 & 2.05 & 7.46 & 1.31 & $\cdots$ & $(\mathrm{s} \mid \mathrm{s})$ & $\cdots$ & & Cohen et al. (2013) \\
\hline HE $1443+0113$ & 5000 & 2.0 & -2.16 & 1.73 & 1.78 & 8.05 & 1.79 & $\cdots$ & $(s \mid s)$ & $\cdots$ & & Cohen et al. (2013) \\
\hline HE $1447+0102$ & 5100 & 1.7 & -2.52 & 2.49 & 2.55 & 8.46 & 2.74 & $\ldots$ & $(\mathrm{s} \mid \mathrm{s})$ & $\cdots$ & & Aoki et al. (2007) \\
\hline HE $1456+0230$ & 5664 & 2.2 & -3.37 & 2.35 & 2.37 & 7.43 & -0.19 & $\cdots$ & ( no $\mid$ s) & $\cdots$ & $\sqrt{ }$ & Cohen et al. (2013) \\
\hline HE 1509-0806 & 5185 & 2.5 & -2.96 & 2.19 & 2.21 & 7.68 & 1.93 & $<0.97$ & $(\mathrm{~s} \mid \mathrm{s})$ & $\cdots$ & & Cohen et al. (2013) \\
\hline HE $1523-1155$ & 4800 & 1.6 & -2.20 & 1.87 & 1.94 & 8.17 & 1.76 & $\cdots$ & $(s \mid s)$ & 2 & & Aoki et al. (2007) \\
\hline HE 1528-0409 & 5000 & 1.8 & -2.65 & 2.42 & 2.48 & 8.26 & 2.34 & $\cdots$ & $\left(\begin{array}{lll}s & s\end{array}\right)$ & $\cdots$ & & Aoki et al. (2007) \\
\hline HE $2122-4707$ & 5147 & 2.5 & -2.47 & 1.81 & 1.83 & 7.79 & 2.03 & 2.05 & $(\mathrm{r} / \mathrm{s} \mid \mathrm{r} / \mathrm{s})$ & $\cdots$ & & Cohen et al. (2013) \\
\hline HE 2133-1426 & 6300 & 4.1 & -3.37 & 2.00 & 2.00 & 7.06 & 2.34 & $\cdots$ & $(\mathrm{s} \mid \mathrm{no})$ & $\cdots$ & $\sqrt{ }$ & Cohen et al. (2013) \\
\hline
\end{tabular}


Table 1 (continued)

\begin{tabular}{|c|c|c|c|c|c|c|c|c|c|c|c|c|}
\hline Name & $\begin{array}{l}\mathrm{T}_{\text {eff }} \\
(\mathrm{K})\end{array}$ & $\begin{array}{l}\log g \\
(\mathrm{cgs})\end{array}$ & {$[\mathrm{Fe} / \mathrm{H}]$} & {$[\mathrm{C} / \mathrm{Fe}]$} & {$[\mathrm{C} / \mathrm{Fe}]_{c}$} & $\begin{array}{c}A(\mathrm{C}) \\
\text { (corrected) }\end{array}$ & {$[\mathrm{Ba} / \mathrm{Fe}]$} & {$[\mathrm{Eu} / \mathrm{Fe}]$} & $\begin{array}{c}\text { Class } \\
(\mathrm{Ba} \mid A(\mathrm{C}))\end{array}$ & Binarity $^{\mathrm{a}}$ & $\begin{array}{l}\text { Interesting } \\
\text { outliers }\end{array}$ & Reference \\
\hline HE 2138-3336 & 5850 & 3.6 & -2.79 & 2.43 & 2.43 & 8.07 & 1.91 & $<1.09$ & $(\mathrm{~s} \mid \mathrm{s})$ & $\cdots$ & & Placco et al. (2013) \\
\hline HE 2144-1832 & 4200 & 0.6 & -1.7 & 0.8 & 1.02 & 7.8 & 1.3 & $\cdots$ & $(\mathrm{s} \mid \mathrm{s})$ & $\cdots$ & & Hansen et al. (2016a) \\
\hline HE $2148-1247$ & 6380 & 3.9 & -2.35 & 2.00 & 2.20 & 8.08 & 2.34 & 2.00 & $(r / s \mid r / s)$ & $\cdots$ & & Cohen et al. (2013) \\
\hline HE 2153-2323 & 4300 & 0.6 & -2.4 & 1.6 & 1.8 & 7.8 & 1.1 & $\cdots$ & $(s \mid s)$ & $\cdots$ & & Hansen et al. (2016a) \\
\hline HE 2155-3750 & 5000 & 2.3 & -2.64 & 1.91 & 1.93 & 7.72 & 1.92 & $<1.69$ & $(\mathrm{~s} \mid \mathrm{s})$ & $\cdots$ & & Cohen et al. (2013) \\
\hline HE 2158-0348 & 5150 & 2.44 & -2.57 & 1.87 & 1.89 & 7.75 & 1.75 & 0.85 & $(\mathrm{~s} \mid \mathrm{s})$ & $\cdots$ & & Yong et al. (2013) \\
\hline HE 2201-0345 & 4890 & 1.92 & -2.80 & 2.30 & 2.36 & 7.99 & 0.62 & $\cdots$ & $(\mathrm{s} \mid \mathrm{s})$ & 2 & & Hansen et al. (2016c) \\
\hline HE $2202-4831$ & 5331 & 2.95 & -2.78 & 2.41 & 2.43 & 8.08 & -1.28 & $\cdots$ & $($ no $\mid \mathrm{s})$ & $\cdots$ & $\sqrt{ }$ & Yong et al. (2013) \\
\hline HE 2208-1239 & 5100 & 2.3 & -2.88 & 1.30 & 1.31 & 6.86 & 1.68 & 1.52 & $(\mathrm{r} / \mathrm{s} \mid \mathrm{no})$ & $\cdots$ & $\sqrt{ }$ & Hansen et al. (2015a) \\
\hline HE 2221-0453 & 4430 & 0.73 & -2.00 & 1.83 & 1.91 & 8.34 & 1.76 & $\cdots$ & $(\mathrm{s} \mid \mathrm{s})$ & $\cdots$ & & Yong et al. (2013) \\
\hline HE $2227-4044$ & 5811 & 3.85 & -2.32 & 1.64 & 1.64 & 7.75 & 1.33 & $\cdots$ & $(\mathrm{s} \mid \mathrm{s})$ & $\cdots$ & & Barklem et al. (2005) \\
\hline HE 2228-0706 & 5003 & 2.02 & -2.78 & 2.32 & 2.36 & 8.01 & 2.46 & $\cdots$ & $(\mathrm{s} \mid \mathrm{s})$ & $\cdots$ & & Yong et al. (2013) \\
\hline HE $2238-4131$ & 5200 & 2.5 & -2.75 & 2.63 & 2.65 & 8.33 & 1.80 & 1.10 & $(\mathrm{~s} \mid \mathrm{s})$ & $\cdots$ & & Hansen et al. (2015a) \\
\hline HE $2240-0412$ & 5852 & 4.33 & -2.20 & 1.31 & 1.31 & 7.54 & 1.32 & $\cdots$ & $(\mathrm{s} \mid \mathrm{s})$ & $\cdots$ & & Barklem et al. (2005) \\
\hline HE 2251-0821 & 5160 & 2.5 & -2.96 & 1.97 & 1.99 & 7.46 & 1.75 & $\cdots$ & $(\mathrm{s} \mid \mathrm{s})$ & $\cdots$ & & Cohen et al. (2013) \\
\hline HE 2258-6358 & 4900 & 1.6 & -2.67 & 2.42 & 2.50 & 8.26 & 2.23 & 1.68 & $\left(\begin{array}{lll}\mathrm{s} \mid & \mathrm{s}\end{array}\right)$ & $\cdots$ & & Placco et al. (2013) \\
\hline HE 2312-0758 & 4827 & 1.26 & -3.47 & 1.86 & 2.25 & 7.21 & 1.99 & $\cdots$ & $(\mathrm{s} \mid \mathrm{s})$ & 2 & $\sqrt{ }$ & Hansen et al. (2016c) \\
\hline HE 2319-5228 & 4900 & 1.6 & -2.6 & 1.7 & 1.9 & 7.7 & $<-3.0$ & $\cdots$ & $($ no $\mid \mathrm{s})$ & $\cdots$ & $\sqrt{ }$ & Hansen et al. (2016a) \\
\hline HE 2330-0555 & 4867 & 1.65 & -2.98 & 2.09 & 2.24 & 7.69 & 1.17 & $\cdots$ & $(\mathrm{s} \mid \mathrm{s})$ & 1 & $\sqrt{ }$ & Yong et al. (2013) \\
\hline HKII 17435-00532 & 5200 & 2.15 & -2.23 & 0.77 & 0.78 & 6.98 & 0.86 & 0.47 & $(\mathrm{r} / \mathrm{s} \mid \mathrm{no})$ & $\cdots$ & $\sqrt{ }$ & Roederer et al. (2008) \\
\hline LP $625-44$ & 5500 & 2.8 & -2.66 & 2.11 & 2.13 & 7.90 & 2.78 & $\cdots$ & $(\mathrm{s} \mid \mathrm{s})$ & 2 & & Masseron et al. (2012) \\
\hline SDSS J0002+2928 & 6150 & 4.0 & -3.26 & 2.63 & 2.63 & 7.80 & 1.84 & $\cdots$ & $(\mathrm{s} \mid \mathrm{s})$ & $\cdots$ & & Aoki et al. (2013) \\
\hline SDSS J0126+0607 & 6900 & 4.0 & -3.01 & 3.08 & 3.08 & 8.50 & 3.20 & $\cdots$ & $(\mathrm{s} \mid \mathrm{s})$ & $\cdots$ & & Aoki et al. (2013) \\
\hline SDSS J0212+0137 & 6333 & 4.0 & -3.57 & 2.26 & 2.26 & 7.12 & 0.51 & $\cdots$ & $($ no $\mid \mathrm{s})$ & $\cdots$ & $\sqrt{ }$ & Bonifacio et al. (2015) \\
\hline SDSS J0912+0216 & 6500 & 4.5 & -2.45 & 2.18 & 2.18 & 8.16 & 1.53 & $\cdots$ & $(\mathrm{s} \mid \mathrm{s})$ & $\cdots$ & & Masseron et al. (2012) \\
\hline SDSS J0924+4059 & 6196 & 3.77 & -2.68 & 2.72 & 2.72 & 8.47 & 1.73 & $\cdots$ & $(\mathrm{s} \mid \mathrm{s})$ & $\cdots$ & & Yong et al. (2013) \\
\hline SDSS J1114+1828 & 6200 & 4.0 & -3.33 & 3.30 & 3.30 & 8.40 & 1.59 & $\cdots$ & $(\mathrm{s} \mid \mathrm{s})$ & $2 ?^{\mathrm{i}}$ & & Spite et al. (2013) \\
\hline SDSS J1137+2553 & 6310 & 3.2 & -2.64 & 2.81 & 2.83 & 8.62 & 2.77 & $\cdots$ & $(\mathrm{s} \mid \mathrm{s})$ & $\cdots$ & & Bonifacio et al. (2015) \\
\hline SDSS J1143+2020 & 6240 & 4.0 & -3.13 & 2.80 & 2.80 & 8.10 & 1.81 & $\cdots$ & $(\mathrm{s} \mid \mathrm{s})$ & $2 ?^{\mathrm{i}}$ & & Spite et al. (2013) \\
\hline SDSS J1245-0738 & 6100 & 4.0 & -3.17 & 2.53 & 2.53 & 7.79 & 2.09 & $\cdots$ & $(\mathrm{s} \mid \mathrm{s})$ & $\cdots$ & & Aoki et al. (2013) \\
\hline SDSS J1349-0229 & 6200 & 4.0 & -2.95 & 2.83 & 2.83 & 8.28 & 2.21 & $\cdots$ & $\left(\begin{array}{ll}\mathrm{s} \mid & \mathrm{s}\end{array}\right)$ & $\cdots$ & & Masseron et al. (2012) \\
\hline SDSS J1422+0031 & 5200 & 2.2 & -3.03 & 1.70 & 1.71 & 7.11 & -1.18 & $\cdots$ & $($ no $\mid \mathrm{s})$ & $\cdots$ & $\sqrt{ }$ & Aoki et al. (2013) \\
\hline SDSS J1613+5309 & 5350 & 2.1 & -3.33 & 2.09 & 2.11 & 7.21 & 0.03 & $\cdots$ & $($ no $\mid \mathrm{s})$ & $\cdots$ & $\sqrt{ }$ & Aoki et al. (2013) \\
\hline SDSS J1626+1458 & 6400 & 4.0 & -2.99 & 2.86 & 2.86 & 8.30 & 1.69 & $\cdots$ & $(\mathrm{s} \mid \mathrm{s})$ & $\cdots$ & & Aoki et al. (2013) \\
\hline SDSS J1646+2824 & 6100 & 4.0 & -3.05 & 2.52 & 2.52 & 7.90 & 1.78 & $\cdots$ & $\left(\begin{array}{ll}s \mid & s\end{array}\right)$ & $\cdots$ & & Aoki et al. (2013) \\
\hline SDSS J1707+5850 & 6700 & 4.2 & -2.57 & 2.14 & 2.14 & 8.00 & 3.44 & $\cdots$ & $(\mathrm{s} \mid \mathrm{s})$ & $\cdots$ & & Aoki et al. (2008) \\
\hline SDSS J1734+4316 & 5200 & 2.7 & -2.51 & 1.78 & 1.80 & 7.72 & 1.61 & $\cdots$ & $(\mathrm{s} \mid \mathrm{s})$ & $\cdots$ & & Aoki et al. (2013) \\
\hline SDSS J1836+6317 & 5350 & 3.0 & -2.85 & 2.02 & 2.03 & 7.61 & 2.37 & $\cdots$ & $(s \mid s)$ & $\cdots$ & & Aoki et al. (2013) \\
\hline SDSS J2047+0015 & 6383 & 4.36 & -2.36 & 2.00 & 2.00 & 8.07 & 1.70 & $\cdots$ & $(\mathrm{s} \mid \mathrm{s})$ & $\cdots$ & & Yong et al. (2013) \\
\hline \multicolumn{13}{|c|}{ Group II Stars } \\
\hline $\mathrm{BD}+44493$ & 5430 & 3.4 & -3.83 & 1.35 & 1.35 & 5.95 & -0.60 & $<0.41$ & ( no $\mid$ no) & 1 & & Ito et al. (2013) \\
\hline BD-01 2582 & 4920 & 1.8 & -2.62 & 0.86 & 1.02 & 6.83 & 1.05 & 0.36 & $(\mathrm{~s} \mid \mathrm{no})$ & $\cdots$ & $\sqrt{ }$ & Roederer et al. (2014b) \\
\hline BD-18 5550 & 4660 & 1.05 & -3.20 & 0.05 & 0.82 & 6.05 & -0.82 & -0.41 & ( no $\mid$ no) & $\cdots$ & & Roederer et al. (2014b) \\
\hline BS 16929-005 & 5229 & 2.61 & -3.34 & 0.99 & 1.00 & 6.09 & -0.41 & $\cdots$ & ( no $\mid$ no) & 1 & & Yong et al. (2013) \\
\hline $\mathrm{CD}-2417504$ & 6228 & 3.90 & -3.41 & 1.10 & 1.10 & 6.12 & $<-1.05$ & $<1.16$ & ( no $\mid$ no) & 2 & $\checkmark$ & Jacobson et al. (2015) \\
\hline CS 22185-007 & 4730 & 1.30 & -3.06 & 0.16 & 0.74 & 6.11 & -0.96 & -0.26 & ( no $\mid$ no) & $\cdots$ & & Roederer et al. (2014b) \\
\hline CS 22189-009 & 4540 & 0.60 & -3.92 & 0.30 & 1.00 & 5.51 & -1.52 & $<0.32$ & ( no $\mid$ no) & $\cdots$ & & Roederer et al. (2014b) \\
\hline CS $22873-128$ & 4710 & 1.20 & -3.32 & 0.12 & 0.79 & 5.90 & -1.37 & $<-0.26$ & ( no $\mid$ no) & $\cdots$ & & Roederer et al. (2014b) \\
\hline CS $22874-123$ & 6240 & 3.80 & -2.79 & 0.72 & 0.72 & 6.36 & -0.13 & $<1.41$ & ( no $\mid$ no) & $\cdots$ & & Roederer et al. (2014b) \\
\hline CS 22877-001 & 4790 & 1.45 & -3.31 & 1.10 & 1.55 & 6.67 & -0.50 & 0.03 & ( no $\mid$ no) & 1 & & Roederer et al. (2014b) \\
\hline CS 22885-096 & 4580 & 0.75 & -4.41 & 0.60 & 1.27 & 5.29 & -1.64 & $<0.68$ & ( no $\mid$ no) & $\cdots$ & & Roederer et al. (2014b) \\
\hline CS 22888-031 & 5810 & 3.50 & -3.71 & 0.92 & 0.92 & 5.64 & $<-0.41$ & $<1.79$ & ( no $\mid$ no) & $\cdots$ & & Roederer et al. (2014b) \\
\hline CS 22891-200 & 4490 & 0.50 & -4.06 & 0.53 & 1.21 & 5.58 & -0.75 & 0.11 & ( no $\mid$ no) & $\cdots$ & & Roederer et al. (2014b) \\
\hline CS 22892-025 & 6140 & 3.75 & -2.78 & 0.76 & 0.76 & 6.41 & -0.36 & $<1.09$ & ( no $\mid$ no) & $\cdots$ & & Roederer et al. (2014b) \\
\hline CS 22896-110 & 5380 & 1.15 & -2.85 & 0.19 & 0.82 & 6.40 & -0.59 & 0.04 & ( no $\mid$ no) & $\cdots$ & & Roederer et al. (2014b) \\
\hline CS 22897-008 & 4550 & 0.70 & -3.83 & 0.60 & 1.32 & 5.92 & -1.17 & -0.03 & ( no $\mid$ no) & $\cdots$ & & Roederer et al. (2014b) \\
\hline CS 22949-037 & 4630 & 0.95 & -4.38 & 1.16 & 1.92 & 5.97 & -0.6 & $<0.69$ & ( no $\mid$ no) & 1 & & Roederer et al. (2014b) \\
\hline CS 22949-048 & 4620 & 0.95 & -3.55 & 0.17 & 0.93 & 5.81 & -1.45 & $<-0.10$ & ( no $\mid$ no) & $\cdots$ & & Roederer et al. (2014b) \\
\hline CS $22950-046$ & 4380 & 0.50 & -4.12 & 0.61 & 1.30 & 5.61 & -1.01 & $<0.23$ & ( no $\mid$ no) & $\cdots$ & & Roederer et al. (2014b) \\
\hline CS 22953-037 & 6150 & 3.70 & -3.21 & 1.00 & 1.00 & 6.22 & $<-0.72$ & $<1.40$ & ( no $\mid$ no) & $\cdots$ & & Roederer et al. (2014b) \\
\hline CS 22956-050 & 4640 & 1.00 & -3.67 & 0.26 & 0.98 & 5.74 & -0.90 & $<-0.09$ & ( no $\mid$ no) & $\cdots$ & & Roederer et al. (2014b) \\
\hline CS 22957-013 & 4620 & 0.95 & -2.98 & -0.01 & 0.72 & 6.17 & -0.83 & -0.31 & ( no $\mid$ no) & $\cdots$ & & Roederer et al. (2014b) \\
\hline CS 22957-024 & 6160 & 3.75 & -2.91 & 0.78 & 0.78 & 6.30 & -0.55 & $<1.14$ & ( no $\mid$ no) & $\cdots$ & & Roederer et al. (2014b) \\
\hline
\end{tabular}


YOON ET AL.

Table 1 (continued)

\begin{tabular}{|c|c|c|c|c|c|c|c|c|c|c|c|c|}
\hline Name & $\begin{array}{l}\mathrm{T}_{\text {eff }} \\
(\mathrm{K}) \\
(2) \\
\end{array}$ & $\begin{array}{c}\log g \\
(\mathrm{cgs}) \\
(3) \\
\end{array}$ & {$[\mathrm{Fe} / \mathrm{H}]$} & {$[\mathrm{C} / \mathrm{Fe}]$} & {$[\mathrm{C} / \mathrm{Fe}]_{c}$} & $\begin{array}{c}A(\mathrm{C}) \\
\text { (corrected) } \\
(7) \\
\end{array}$ & {$[\mathrm{Ba} / \mathrm{Fe}]$} & {$[\mathrm{Eu} / \mathrm{Fe}]$} & $\begin{array}{c}\text { Class } \\
(\mathrm{Ba} \mid A(\mathrm{C})) \\
(10) \\
\end{array}$ & $\begin{array}{c}\text { Binarity }^{\mathrm{a}} \\
\text { (11) } \\
\end{array}$ & $\begin{array}{c}\text { Interesting } \\
\text { outliers } \\
(12) \\
\end{array}$ & Reference \\
\hline CS 22958-083 & 4900 & 1.75 & -3.05 & 0.53 & 0.71 & 6.09 & -0.99 & $<-0.11$ & ( no $\mid$ no) & $\cdots$ & & Roederer et al. (2014b) \\
\hline CS $22960-048$ & 4770 & 1.40 & -3.91 & 0.47 & 0.93 & 5.45 & -1.59 & $<0.55$ & ( no $\mid$ no) & $\cdots$ & & Roederer et al. (2014b) \\
\hline CS $22960-053^{f}$ & 4860 & 1.65 & -3.64 & 1.40 & 1.64 & 6.43 & 1.03 & $<0.70$ & $(\mathrm{~s} \mid \mathrm{no})$ & $\cdots$ & $\sqrt{ }$ & Roederer et al. (2014b) \\
\hline CS $22964-183$ & 6010 & 3.65 & -3.01 & 0.83 & 0.83 & 6.25 & -0.92 & $<1.24$ & ( no $\mid$ no) & $\cdots$ & & Roederer et al. (2014b) \\
\hline CS 22964-214 & 6180 & 3.75 & -2.95 & 0.73 & 0.73 & 6.21 & -0.43 & $<1.24$ & ( no $\mid$ no) & $\cdots$ & & Roederer et al. (2014b) \\
\hline CS 22965-054 & 6050 & 3.70 & -3.17 & 1.02 & 1.02 & 6.28 & $<-0.5$ & $<1.73$ & ( no $\mid$ no) & $\cdots$ & & Roederer et al. (2014b) \\
\hline CS 29493-050 & 6270 & 3.80 & -2.92 & 0.84 & 0.84 & 6.35 & -0.69 & $<1.36$ & ( no $\mid$ no) & $\cdots$ & & Roederer et al. (2014b) \\
\hline CS 29502-092 & 4820 & 1.50 & -3.30 & 1.06 & 1.46 & 6.59 & -1.36 & $<0.11$ & ( no $\mid$ no) & 1 & & Roederer et al. (2014b) \\
\hline CS 29504-006 & 6150 & 3.70 & -3.12 & 1.59 & 1.59 & 6.90 & 0.41 & $<1.75$ & ( no $\mid$ no) & $\cdots$ & & Roederer et al. (2014b) \\
\hline CS 29514-007 & 6400 & 3.85 & -2.83 & 0.89 & 0.89 & 6.49 & -0.14 & $<1.74$ & ( no $\mid$ no) & $\cdots$ & & Roederer et al. (2014b) \\
\hline CS 30314-067 & 4320 & 0.50 & -3.31 & 0.85 & 1.58 & 6.70 & -0.25 & -0.32 & ( no $\mid$ no) & $\cdots$ & & Roederer et al. (2014b) \\
\hline CS $30492-110$ & 4660 & 1.05 & -3.27 & 0.12 & 0.88 & 6.04 & -0.43 & 0.28 & ( no $\mid$ no) & $\cdots$ & & Roederer et al. (2014b) \\
\hline HD 126587 & 4640 & 1.0 & -3.29 & 0.06 & 0.84 & 5.98 & -0.25 & 0.24 & ( no $\mid$ no) & $\cdots$ & & Roederer et al. (2014b) \\
\hline HD 237846 & 4730 & 1.3 & -3.21 & 0.15 & 0.74 & 5.96 & -0.92 & -0.35 & ( no $\mid$ no) & $\cdots$ & & Roederer et al. (2014b) \\
\hline HE $0015+0048$ & 4600 & 0.9 & -3.07 & 0.62 & 1.29 & 6.65 & -1.17 & $\cdots$ & ( no $\mid$ no) & $\cdots$ & & Hollek et al. (2011) \\
\hline HE $0020-1741$ & 4765 & 1.55 & -4.05 & 1.40 & 1.74 & 6.12 & -1.11 & $<0.25$ & ( no $\mid$ no) & 1 & & Placco et al. (2016) \\
\hline HE $0055-2314$ & 6290 & 4.4 & -2.70 & 0.93 & 0.93 & 6.66 & -0.49 & $\cdots$ & ( no $\mid$ no) & $\cdots$ & & Cohen et al. (2013) \\
\hline HE 0057-5959 & 5257 & 2.65 & -4.08 & 0.86 & 0.86 & 5.21 & -0.46 & $\cdots$ & ( no $\mid$ no) & $\cdots$ & & Yong et al. (2013) \\
\hline HE $0102-1213$ & 6100 & 3.65 & -3.28 & 1.31 & 1.31 & 6.46 & $<-0.64$ & $\cdots$ & ( no $\mid$ no) & $\cdots$ & & Yong et al. (2013) \\
\hline HE 0103-0352 & 5406 & 3.2 & -3.20 & 0.73 & 0.73 & 5.96 & -0.87 & $\cdots$ & ( no $\mid$ no) & $\cdots$ & & Cohen et al. (2013) \\
\hline HE 0130-1749 & 4820 & 1.6 & -3.39 & 0.61 & 0.90 & 5.94 & -0.72 & $<0.65$ & ( no $\mid$ no) & $\cdots$ & & Cohen et al. (2013) \\
\hline HE $0132-2429$ & 5294 & 2.75 & -3.6 & 0.83 & 0.84 & 5.67 & -0.85 & $<1.22$ & ( no $\mid$ no) & $\cdots$ & & Cohen et al. (2008) \\
\hline HE 0134-1519 & 5525 & 3.17 & -3.98 & 1.00 & 1.00 & 5.45 & $<-0.50$ & $<1.50$ & ( no $\mid$ no) & $\cdots$ & & Hansen et al. (2014) \\
\hline HE $0139-2826$ & 4900 & 1.5 & -3.46 & 0.48 & 0.86 & 5.83 & -1.22 & $<0.21$ & ( no $\mid$ no) & $\cdots$ & & Placco et al. (2014a) \\
\hline HE $0146-1548$ & 4636 & 0.99 & -3.46 & 0.84 & 1.57 & 6.54 & -0.71 & $\cdots$ & ( no $\mid$ no) & $\cdots$ & & Yong et al. (2013) \\
\hline HE $0302-3417 \mathrm{~A}$ & 4400 & 0.2 & -3.70 & 0.48 & 1.20 & 5.93 & -2.10 & $\cdots$ & ( no $\mid$ no) & $\cdots$ & & Hollek et al. (2011) \\
\hline HE $0324+0152 \mathrm{~A}$ & 4775 & 1.2 & -3.32 & 0.18 & 0.84 & 5.95 & -1.20 & $<0.66$ & ( no $\mid$ no) & $\cdots$ & & Hollek et al. (2011) \\
\hline HE 0401-3835 & 5458 & 3.2 & -3.10 & 0.99 & 0.99 & 6.32 & -0.75 & $<0.88$ & ( no $\mid$ no) & $\cdots$ & & Cohen et al. (2013) \\
\hline $\mathrm{HE} 0420+0123 \mathrm{~A}$ & 4800 & 1.45 & -3.03 & 0.33 & 0.78 & 6.18 & 0.08 & $\cdots$ & ( no $\mid$ no) & $\cdots$ & & Hollek et al. (2011) \\
\hline HE $0432-1005 \mathrm{~A}$ & 4525 & 0.5 & -3.21 & 0.23 & 0.96 & 6.18 & -0.90 & $\cdots$ & ( no $\mid$ no) & $\cdots$ & & Hollek et al. (2011) \\
\hline HE 0440-1049 & 5800 & 3.5 & -3.02 & 0.69 & 0.69 & 6.10 & -1.27 & $<1.50$ & ( no $\mid$ no) & $\cdots$ & & Hansen et al. (2015a) \\
\hline HE $0557-4840$ & 4900 & 2.2 & -4.73 & 1.59 & 1.60 & 5.29 & $<0.00$ & $<2.02$ & ( no $\mid$ no) & $\cdots$ & & Norris et al. (2007) \\
\hline HE 1116-0634 & 4400 & 0.1 & -3.73 & 0.08 & 0.81 & 5.51 & -1.81 & $\cdots$ & ( no $\mid$ no) & $\cdots$ & & Hollek et al. (2011) \\
\hline HE 1124-2335 & 4870 & 1.65 & -3.36 & 0.74 & 1.00 & 6.07 & -1.19 & $<0.13$ & ( no $\mid$ no) & $\cdots$ & & Roederer et al. (2014b) \\
\hline HE 1201-1512 & 5725 & 3.39 & -3.92 & 1.60 & 1.60 & 6.11 & $<-0.34$ & $\cdots$ & ( no $\mid$ no) & 1 & & Yong et al. (2013) \\
\hline HE $1300+0157$ & 5529 & 3.25 & -3.75 & 1.31 & 1.31 & 5.99 & $<-0.85$ & $\cdots$ & ( no $\mid$ no) & 1 & & Yong et al. (2013) \\
\hline HE $1300-0641$ & 5308 & 2.96 & -3.14 & 1.25 & 1.25 & 6.54 & -0.82 & $\cdots$ & ( no $\mid$ no) & 1 & & Barklem et al. (2005) \\
\hline HE $1300-2201$ & 6332 & 4.64 & -2.60 & 0.98 & 0.98 & 6.80 & -0.09 & $\cdots$ & ( no $\mid$ no) & $\cdots$ & & Barklem et al. (2005) \\
\hline HE 1311-0131 & 4825 & 1.5 & -3.15 & 0.33 & 0.73 & 6.01 & -0.62 & & ( no $\mid$ no) & $\cdots$ & & Hollek et al. (2011) \\
\hline HE 1330-0354 & 6257 & 4.13 & -2.29 & 1.01 & 1.01 & 7.15 & -0.52 & $\cdots$ & ( no $\mid$ no) & $\cdots$ & & Barklem et al. (2005) \\
\hline HE 1338-0052 & 5856 & 3.7 & -3.06 & 1.53 & 1.53 & 6.90 & -0.02 & $<1.50$ & ( no $\mid$ no) & $\cdots$ & & Cohen et al. (2013) \\
\hline HE 1351-1049 & 5204 & 2.85 & -3.45 & 1.52 & 1.53 & 6.50 & 0.09 & $\cdots$ & ( no $\mid$ no) & $\cdots$ & & Barklem et al. (2005) \\
\hline HE $1405-2512$ & 5602 & 3.3 & -2.85 & 0.73 & 0.73 & 6.31 & -1.15 & $\cdots$ & ( no $\mid$ no) & $\cdots$ & & Cohen et al. (2013) \\
\hline HE $1432-1819$ & 5975 & 3.6 & -2.61 & 1.04 & 1.04 & 6.86 & -0.67 & $\cdots$ & ( no $\mid$ no) & $\cdots$ & & Cohen et al. (2013) \\
\hline HE $1439-0218$ & 6150 & 3.7 & -2.70 & 0.87 & 0.87 & 6.60 & -0.15 & $\cdots$ & ( no $\mid$ no) & $\cdots$ & & Cohen et al. (2013) \\
\hline HE 1506-0113 & 5016 & 2.01 & -3.54 & 1.47 & 1.49 & 6.38 & -0.80 & $\cdots$ & ( no $\mid$ no) & 2 & $\sqrt{ }$ & Yong et al. (2013) \\
\hline HE 2123-0329 & 4725 & 1.15 & -3.22 & 0.40 & 1.06 & 6.27 & -0.85 & $\cdots$ & ( no $\mid$ no) & $\cdots$ & & Hollek et al. (2011) \\
\hline HE 2138-0314 & 5015 & 1.9 & -3.29 & 0.78 & 0.82 & 5.96 & -0.85 & $\cdots$ & ( no $\mid$ no) & $\cdots$ & & Hollek et al. (2011) \\
\hline HE 2141-3741 & 4945 & 1.0 & -3.3 & 0.07 & 0.83 & 5.96 & -1.28 & $<-0.17$ & ( no $\mid$ no) & $\cdots$ & & Placco et al. (2014a) \\
\hline HE $2142-5656$ & 4939 & 1.85 & -2.87 & 0.95 & 1.05 & 6.61 & -0.63 & $\cdots$ & ( no $\mid$ no) & $\cdots$ & & Yong et al. (2013) \\
\hline HE 2235-5058 & 5200 & 2.5 & -2.7 & 1.0 & 1.0 & 6.8 & 2.4 & $\cdots$ & $(\mathrm{s} \mid \mathrm{no})$ & $\cdots$ & $\checkmark$ & Hansen et al. (2016a) \\
\hline HE 2247-7400 & 4829 & 1.56 & -2.87 & 0.70 & 1.02 & 6.58 & -0.94 & $\cdots$ & ( no $\mid$ no) & $\cdots$ & & Yong et al. (2013) \\
\hline HE $2250-4229$ & 5200 & 2.4 & -2.7 & 0.9 & 0.9 & 6.7 & -0.3 & $\cdots$ & ( no $\mid$ no) & $\cdots$ & & Hansen et al. (2016a) \\
\hline HE $2302-2154 \mathrm{~A}$ & 4675 & 0.9 & -3.90 & 0.38 & 1.08 & 5.61 & -1.50 & $\cdots$ & ( no $\mid$ no) & $\cdots$ & & Hollek et al. (2011) \\
\hline HE 2314-1554 & 5050 & 2.2 & -3.33 & 0.78 & 0.79 & 5.89 & -0.49 & $\cdots$ & ( no $\mid$ no) & $\cdots$ & & Cohen et al. (2013) \\
\hline HE 2318-1621 & 4846 & 1.4 & -3.67 & 1.04 & 1.54 & 6.3 & -1.61 & $<0.13$ & ( no $\mid$ no) & 1 & & Placco et al. (2014a) \\
\hline HE 2323-6549 & 5215 & 2.6 & -3.35 & 0.72 & 0.73 & 5.81 & -0.58 & $<0.58$ & ( no $\mid$ no) & $\cdots$ & & Placco et al. (2014a) \\
\hline HE 2331-7155 & 4900 & 1.5 & -3.68 & 1.34 & 1.72 & 6.47 & -0.90 & $<0.50$ & ( no $\mid$ no) & $\cdots$ & & Hansen et al. (2015a) \\
\hline LAMOST J1253+0753 & 6030 & 3.65 & -4.02 & 1.59 & 1.59 & 6.00 & $<-0.30$ & $<1.60$ & ( no $\mid$ no) & $\cdots$ & & Li et al. (2015) \\
\hline SDSS J0140+2344 & 5703 & 3.36 & -4.09 & 1.57 & 1.57 & 5.91 & $<-0.04$ & $\cdots$ & ( no $\mid$ no) & $\cdots$ & & Yong et al. (2013) \\
\hline SDSS J0741+6708 & 5200 & 2.5 & -2.87 & 0.74 & 0.75 & 6.31 & 0.26 & $\cdots$ & ( no $\mid$ no) & $\cdots$ & & Aoki et al. (2013) \\
\hline SDSS J1036+1212 & 6000 & 4.0 & -3.15 & 1.48 & 1.48 & 6.76 & 1.21 & $\cdots$ & $(\mathrm{s} \mid \mathrm{no})$ & $\cdots$ & $\sqrt{ }$ & Masseron et al. (2012) \\
\hline SMSS J0021-4711 & 4765 & 1.4 & -3.17 & 0.29 & 0.78 & 6.04 & -1.18 & $<0.50$ & ( no $\mid$ no) & $\cdots$ & & Jacobson et al. (2015) \\
\hline
\end{tabular}


Table 1 (continued)

\begin{tabular}{|c|c|c|c|c|c|c|c|c|c|c|c|c|}
\hline Name & $\begin{array}{c}\mathrm{T}_{\text {eff }} \\
(\mathrm{K}) \\
(2) \\
\end{array}$ & $\begin{array}{c}\log g \\
(\mathrm{cgs}) \\
(3) \\
\end{array}$ & {$[\mathrm{Fe} / \mathrm{H}]$} & {$[\mathrm{C} / \mathrm{Fe}]$} & {$[\mathrm{C} / \mathrm{Fe}]_{c}$} & $\begin{array}{c}A(\mathrm{C}) \\
\text { (corrected) } \\
(7) \\
\end{array}$ & {$[\mathrm{Ba} / \mathrm{Fe}]$} & $\begin{array}{c}{[\mathrm{Eu} / \mathrm{Fe}]} \\
(9) \\
\end{array}$ & $\begin{array}{c}\text { Class } \\
(\mathrm{Ba} \mid A(\mathrm{C})) \\
(10)\end{array}$ & $\begin{array}{c}\text { Binarity }^{a} \\
(11) \\
\end{array}$ & $\begin{array}{c}\text { Interesting } \\
\text { outliers } \\
(12) \\
\end{array}$ & $\begin{array}{c}\text { Reference } \\
\text { (13) } \\
\end{array}$ \\
\hline SMSS J0106-5244 & 4486 & 0.65 & -3.79 & 0.13 & 0.85 & 5.49 & -1.64 & $\cdots$ & ( no $\mid$ no) & $\cdots$ & & Jacobson et al. (2015) \\
\hline SMSS J0231-5239 & 4882 & 1.55 & -2.94 & 0.40 & 0.77 & 6.26 & -0.27 & $<0.00$ & ( no $\mid$ no) & $\cdots$ & & Jacobson et al. (2015) \\
\hline SMSS J0231-5753 & 4720 & 1.05 & -3.42 & 0.35 & 1.07 & 6.08 & -1.63 & $<0.30$ & ( no $\mid$ no) & $\cdots$ & & Jacobson et al. (2015) \\
\hline SMSS J0330-6813 & 4882 & 1.45 & -3.44 & 0.65 & 1.08 & 6.07 & -0.68 & $<0.62$ & ( no $\mid$ no) & $\cdots$ & & Jacobson et al. (2015) \\
\hline SMSS J0617-6007 & 4810 & 1.35 & -2.72 & 0.22 & 0.74 & 6.45 & -0.35 & 0.10 & ( no $\mid$ no) & $\cdots$ & & Jacobson et al. (2015) \\
\hline SMSS J0702-6004 & 4824 & 1.4 & -2.62 & 0.42 & 0.87 & 6.68 & -0.31 & $<0.05$ & ( no $\mid$ no) & $\cdots$ & & Jacobson et al. (2015) \\
\hline SMSS J1232-0545 & 4792 & 1.25 & -3.03 & 0.18 & 0.78 & 6.18 & -0.51 & $<0.30$ & ( no $\mid$ no) & $\cdots$ & & Jacobson et al. (2015) \\
\hline SMSS J1258-3350 & 5040 & 1.8 & -3.44 & 0.61 & 0.71 & 5.70 & -1.02 & $<0.62$ & ( no $\mid$ no) & $\cdots$ & & Jacobson et al. (2015) \\
\hline SMSS J1435-4203 & 4657 & 1.0 & -3.15 & 0.27 & 0.98 & 6.26 & -0.96 & $<-0.07$ & ( no $\mid$ no) & $\cdots$ & & Jacobson et al. (2015) \\
\hline SMSS J1556-1655 & 4837 & 1.45 & -2.79 & 0.36 & 0.80 & 6.44 & -0.40 & $<0.15$ & ( no $\mid$ no) & $\cdots$ & & Jacobson et al. (2015) \\
\hline SMSS J1738-1457 & 4558 & 0.75 & -3.58 & 0.60 & 1.33 & 6.18 & -0.25 & $<0.09$ & ( no $\mid$ no) & $\cdots$ & & Jacobson et al. (2015) \\
\hline SMSS J1749-4551 & 4797 & 1.3 & -2.77 & 0.30 & 0.84 & 6.50 & -0.29 & 0.12 & ( no $\mid$ no) & $\cdots$ & & Jacobson et al. (2015) \\
\hline SMSS J1848-3059 & 4518 & 0.75 & -3.65 & 0.25 & 0.97 & 5.75 & -1.58 & $<0.28$ & ( no $\mid$ no) & $\cdots$ & & Jacobson et al. (2015) \\
\hline SMSS J1905-2149 & 4855 & 1.55 & -3.11 & 0.35 & 0.71 & 6.03 & -1.06 & $<0.24$ & ( no $\mid$ no) & $\cdots$ & & Jacobson et al. (2015) \\
\hline SMSS J2258-2654 & 4612 & 0.8 & -3.49 & 0.36 & 1.09 & 6.03 & -1.13 & $<0.67$ & ( no $\mid$ no $)$ & $\cdots$ & & Jacobson et al. (2015) \\
\hline \multicolumn{13}{|c|}{ Group III Stars } \\
\hline G $77-61$ & 4000 & 5.05 & -4.08 & 2.65 & 2.65 & 7.00 & $<1.00$ & $<3.04$ & ( no $\mid$ no) & $2^{\mathrm{j}}$ & $\sqrt{ }$ & Plez \& Cohen (2005) \\
\hline HE 0107-5240 & 5100 & 2.2 & -5.44 & 3.97 & 4.03 & 7.03 & $<0.93$ & $<2.93$ & ( no $\mid$ no) & $\cdots$ & & Christlieb et al. (2004) \\
\hline HE $0233-0343$ & 6075 & 3.4 & -4.68 & 3.48 & 3.48 & 7.23 & $<0.80$ & $<3.00$ & $($ no $\mid$ s) & $\cdots$ & $\sqrt{ }$ & Hansen et al. (2014) \\
\hline HE $1012-1540$ & 5230 & 2.65 & -4.17 & 2.40 & 2.41 & 6.67 & -0.28 & $<1.60$ & ( no $\mid$ no) & 1 & & Roederer et al. (2014b) \\
\hline HE $1310-0536$ & 4975 & 1.92 & -4.15 & 2.36 & 2.44 & 6.72 & -0.50 & $<1.20$ & ( no $\mid$ no) & $\cdots$ & & Hansen et al. (2014) \\
\hline HE $1327-2326$ & 6180 & 3.7 & -5.71 & 4.18 & 4.18 & 6.90 & $<1.39$ & $<4.43$ & ( no $\mid$ no) & 1 & & Frebel et al. (2008) \\
\hline HE 2139-5432 & 5416 & 3.04 & -4.02 & 2.59 & 2.60 & 7.01 & $<-0.33$ & $\cdots$ & ( no $\mid$ no) & $\cdots$ & & Yong et al. (2013) \\
\hline SDSS J1035+0641 & 6262 & 4.0 & $<-5.05$ & $>3.52$ & $>3.52$ & $>6.90$ & $?^{\mathrm{k}}$ & $\cdots$ & ( no $\mid$ no) & $\cdots$ & & Bonifacio et al. (2015) \\
\hline SDSS J1313-0019 & 5200 & 2.6 & -5.00 & 2.96 & 2.98 & 6.41 & $<0.22$ & $\cdots$ & ( no $\mid$ no) & $\cdots$ & & Frebel et al. (2015) \\
\hline SDSS J1742+2531 & 6345 & 4.0 & -4.78 & 3.61 & 3.61 & 7.26 & $<1.65$ & $\cdots$ & $($ no $\mid$ s) & $\cdots$ & $\sqrt{ }$ & Bonifacio et al. (2015) \\
\hline SDSS J2209-0028 & 6440 & 4.0 & -3.94 & 2.54 & 2.54 & 7.10 & $<1.02$ & $\cdots$ & ( no $\mid$ no) & $\cdots$ & & Spite et al. (2013) \\
\hline SMSS J0313-6708 & 5125 & 2.3 & $<-7.80$ & $>5.39$ & $>5.39$ & $>6.02$ & $?^{1}$ & $?^{1}$ & ( no $\mid$ no) & $\ldots$ & & Bessell et al. (2015) \\
\hline \multicolumn{13}{|c|}{ Unclassified CEMP Stars based on the $[\mathrm{Ba} / \mathrm{Fe}]$ Criterion } \\
\hline CS 22882-012 & 6290 & 3.8 & -2.76 & 1.11 & 1.11 & 6.78 & 0.62 & $<1.24$ & $(-\mid$ no $)$ & $\cdots$ & & Roederer et al. (2014b) \\
\hline CS 29493-090 & 4692 & 1.28 & -3.13 & 0.7 & 1.23 & 6.53 & 0.52 & $\cdots$ & $(-\mid$ no $)$ & $\cdots$ & & Barklem et al. (2005) \\
\hline CS $29527-015^{\mathrm{m}}$ & 6577 & 3.89 & -3.32 & 1.18 & 1.18 & 6.29 & $\cdots$ & $\cdots$ & $(-\mid$ no $)$ & 1 & & Yong et al. (2013) \\
\hline CS 29528-041 & 6150 & 4 & -3.3 & 1.57 & 1.57 & 6.7 & 0.89 & $\cdots$ & $(-\mid$ no $)$ & $\cdots$ & & Sivarani et al. (2006) \\
\hline CS 31080-095 & 6050 & 4.5 & -2.85 & 2.67 & 2.67 & 8.25 & 0.72 & $\cdots$ & $\left(\begin{array}{ll}-1 & s\end{array}\right)$ & $\cdots$ & & Sivarani et al. (2006) \\
\hline HD 135148 & 4183 & 1.24 & -2.17 & 0.76 & 1.10 & 7.36 & $\cdots$ & 0.7 & $\left(\begin{array}{ll}-1 & s\end{array}\right)$ & $\cdots$ & & Simmerer et al. (2004) \\
\hline HE $0440-3426$ & 4800 & 1.6 & -2.19 & 1.51 & 1.63 & 7.87 & 0.46 & $<0.62$ & $\left(\begin{array}{ll}-1 & s\end{array}\right)$ & $\cdots$ & & Hansen et al. (2015a) \\
\hline HE $0450-4705$ & 5429 & 3.34 & -3.10 & 0.81 & 0.81 & 6.14 & $\cdots$ & $\cdots$ & $(-\mid$ no $)$ & $\cdots$ & & Barklem et al. (2005) \\
\hline HE $0516-2515$ & 4400 & 0.7 & -2.5 & $>0.8$ & 1.27 & 7.2 & 0.5 & $\cdots$ & $\left(\begin{array}{ll}-1 & s\end{array}\right)$ & $\cdots$ & & Hansen et al. (2016c) \\
\hline HE 1029-0546 & 6650 & 4.3 & -3.28 & 2.64 & 2.64 & 7.79 & 0.8 & $<2.5$ & $\left(\begin{array}{ll}-1 & s\end{array}\right)$ & $\cdots$ & & Hansen et al. (2015a) \\
\hline HE $1135-0344$ & 6154 & 4.03 & -2.63 & 1.00 & 1.00 & 6.80 & $\cdots$ & $\cdots$ & $(-\mid$ no $)$ & $\cdots$ & & Barklem et al. (2005) \\
\hline HE 1148-0037 & 5964 & 4.16 & -3.47 & 0.80 & 0.80 & 5.76 & $\cdots$ & $\cdots$ & $(-\mid$ no $)$ & $\cdots$ & & Barklem et al. (2005) \\
\hline HE 1217-0540 & 5700 & 4.2 & -2.94 & 0.77 & 0.77 & 6.26 & $\cdots$ & $\cdots$ & $(-\mid$ no $)$ & $\cdots$ & & Barklem et al. (2005) \\
\hline HE 1221-1948 & 6083 & 3.81 & -3.36 & 1.39 & 1.39 & 6.46 & $\cdots$ & $\cdots$ & $(-\mid$ no $)$ & $\cdots$ & & Barklem et al. (2005) \\
\hline HE $1245-0215$ & 6500 & 3.8 & -2.90 & 1.87 & 1.87 & 7.40 & 0.29 & $\cdots$ & $\left(\begin{array}{ll}-1 & s\end{array}\right)$ & $\cdots$ & & Cohen et al. (2013) \\
\hline HE $1245-1616$ & 6191 & 4.04 & -2.97 & 0.73 & 0.73 & 6.19 & 0.23 & & $\left(\begin{array}{ll}-1 & s\end{array}\right)$ & $\cdots$ & & Barklem et al. (2005) \\
\hline HE $1249-3121$ & 5373 & 3.4 & -3.23 & 1.82 & 1.82 & 7.02 & $\cdots$ & $\cdots$ & $(-\mid$ no $)$ & $\cdots$ & & Barklem et al. (2005) \\
\hline HE 1305-0331 & 6081 & 4.22 & -3.26 & 1.09 & 1.09 & 6.26 & $\cdots$ & $\cdots$ & $(-\mid$ no $)$ & $\cdots$ & & Barklem et al. (2005) \\
\hline HE 1413-1954 & 6533 & 4.59 & -3.19 & 1.41 & 1.41 & 6.65 & $\cdots$ & $\cdots$ & $(-\mid$ no $)$ & $\cdots$ & & Barklem et al. (2005) \\
\hline HE $2155-2043^{n}$ & 5200 & 2.4 & -3.0 & 0.70 & 0.71 & 6.14 & $\cdots$ & $\cdots$ & $(-\mid$ no $)$ & $\cdots$ & & Hansen et al. (2016a) \\
\hline SDSS J0036-1043 & 6479 & 4.31 & -2.60 & 2.32 & 2.32 & 8.15 & 0.4 & $\cdots$ & $\left(\begin{array}{ll}-1 & s\end{array}\right)$ & $\cdots$ & & Yong et al. (2013) \\
\hline SDSS J0308+0505 & 5950 & 4.0 & -2.19 & 2.36 & 2.36 & 8.60 & $\cdots$ & $\cdots$ & $\left(\begin{array}{ll}-1 & s\end{array}\right)$ & $\cdots$ & & Aoki et al. (2013) \\
\hline SDSS J0351+1026 & 5450 & 3.6 & -3.18 & 1.55 & 1.55 & 6.80 & $\cdots$ & $\cdots$ & $(-\mid$ no $)$ & $\cdots$ & & Aoki et al. (2013) \\
\hline SDSS J0629+8303 & 5550 & 4.0 & -2.82 & 2.09 & 2.09 & 7.70 & $\cdots$ & $\cdots$ & $\left(\begin{array}{ll}-1 & s\end{array}\right)$ & $\cdots$ & & Aoki et al. (2013) \\
\hline SDSS J0723+3637 & 5150 & 2.2 & -3.32 & 1.79 & 1.81 & 6.92 & $\cdots$ & $\cdots$ & $(-\mid$ no $)$ & $\cdots$ & & Aoki et al. (2013) \\
\hline SDSS J0711+6702 & 5350 & 3 & -2.91 & 1.98 & 1.99 & 7.51 & 0.82 & & $\left(\begin{array}{ll}-1 & s\end{array}\right)$ & $\cdots$ & & Yong et al. (2013) \\
\hline SDSS J0817+2641 & 6300 & 4 & -3.16 & 2.41 & 2.41 & 7.68 & 0.77 & $\cdots$ & $\left(\begin{array}{ll}-1 & s\end{array}\right)$ & $\cdots$ & & Aoki et al. (2008) \\
\hline SDSS J0929+0238 & 5894 & 3.7 & $<-3.81$ & 4.39 & 4.39 & 7.70 & $\cdots$ & $\cdots$ & $\left(\begin{array}{ll}-1 & s\end{array}\right)$ & $\cdots$ & & Bonifacio et al. (2015) \\
\hline SDSS J1746+2455 & 5350 & 2.6 & -3.17 & 1.24 & 1.25 & 6.51 & 0.26 & $\cdots$ & $(-\mid$ no $)$ & $\cdots$ & & Yong et al. (2013) \\
\hline SMSS J0224-5346 & 4630 & 0.9 & -3.4 & 0.03 & 0.78 & 5.81 & 0.38 & $<0.35$ & $(-\mid$ no $)$ & $\cdots$ & & Jacobson et al. (2015) \\
\hline SMSS J0246-3525 & 4720 & 1.05 & -3.37 & 0.02 & 0.77 & 5.83 & 0.26 & $<0.5$ & $(-\mid$ no $)$ & $\cdots$ & & Jacobson et al. (2015) \\
\hline
\end{tabular}


YOON ET AL.

Table 1 (continued)

\begin{tabular}{|c|c|c|c|c|c|c|c|c|c|c|c|c|}
\hline Name & $\begin{array}{l}\mathrm{T}_{\text {eff }} \\
(\mathrm{K})\end{array}$ & $\begin{array}{l}\log g \\
(\mathrm{cgs})\end{array}$ & {$[\mathrm{Fe} / \mathrm{H}]$} & {$[\mathrm{C} / \mathrm{Fe}]$} & {$[\mathrm{C} / \mathrm{Fe}]_{c}$} & $\begin{array}{c}A(\mathrm{C}) \\
\text { (corrected) }\end{array}$ & {$[\mathrm{Ba} / \mathrm{Fe}]$} & {$[\mathrm{Eu} / \mathrm{Fe}]$} & $\begin{array}{c}\text { Class } \\
(\mathrm{Ba} \mid A(\mathrm{C}))\end{array}$ & Binarity $^{a}$ & $\begin{array}{c}\text { Interesting } \\
\text { outliers }\end{array}$ & Reference \\
\hline (1) & (2) & (3) & (4) & (5) & (6) & (7) & (8) & (9) & (10) & (11) & (12) & (13) \\
\hline
\end{tabular}

Note-Stars in the overlapping regions: BD-01 2582 (I/II), CS 22877-001 (II/III), CS 22960-053 (II/III), CS 29502-092 (II/III), CS 29504-006 (I/II/III), CS 30314-067 (II/III), HE 0015+0048 (II/III), HE 0102-1213 (II/III), HE 0146-1548 (II/III), HE 0430-1609 (I/II/III), HE 1300-0641 (II/III), HE 1351-1049 (II/III), HE 1338-0052 (I/II/III), HE 1506-0113 (II/III), HE 2133-1426 (I/III), HE 2208-1239 (I/II/III
SDSS J1036+1212 (II/III), SDSS J0212+0137 (I/III), SDSS J1422+0031 (I/II/III), and SDSS J1613+5309 (I/III).

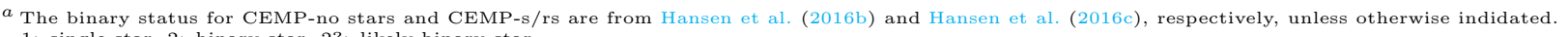
1: single star, 2: binary star, 2?: likely binary star

${ }^{b}$ Binary status is from Preston \& Sneden (2001).

${ }^{c}$ Binary status is from Thompson et al. (2008), recognized as a double-lined spectroscopic binary.

${ }^{d}$ Binary status is from McClure \& Woodsworth (1990).

e Binary status is from Placco et al. (2015).

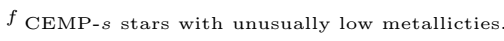

$g$ This star, HE $1105+0027$, was mis-classified as an $r$-II star in Hansen et al. (2015b), which showed this star is a single star.

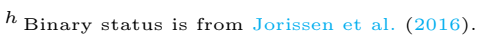

${ }^{i}$ Binary status is from Spite et al. (2013).

$j$ Binary status is from Dearborn et al. (1986)

${ }^{k}$ This star has $[\mathrm{Fe} / \mathrm{H}]<-5.05$ and $A(\mathrm{Ba})<-0.49$, so $[\mathrm{Ba} / \mathrm{Fe}]$ cannot be determined.

$l$ This star has $[\mathrm{Fe} / \mathrm{H}]<-7.8,[\mathrm{Ba} / \mathrm{H}]<-6.1$, and $[\mathrm{Eu} / \mathrm{H}]<-2.9$, so $[\mathrm{Ba} / \mathrm{Fe}]$ and $[\mathrm{Eu} / \mathrm{Fe}]$ cannot be determined.

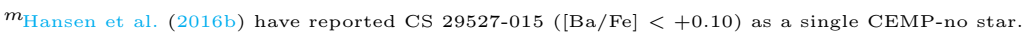

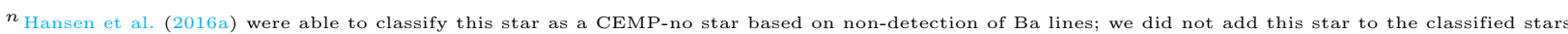
due to the absence of $[\mathrm{Ba} / \mathrm{Fe}]$. However, our classification scheme based on $A(\mathrm{C})$ is consistent with their classification. 
Table 2. Na and Mg abundances for the Group II and Group III CEMP-no stars

\begin{tabular}{|c|c|c|c|c|c|c|c|}
\hline Name & {$[\mathrm{Fe} / \mathrm{H}]$} & $A(\mathrm{C})$ & {$[\mathrm{Na} / \mathrm{Fe}]$} & $A(\mathrm{Na})$ & {$[\mathrm{Mg} / \mathrm{Fe}]$} & $A(\mathrm{Mg})$ & Reference \\
\hline \multicolumn{8}{|c|}{ Group II CEMP-no Stars } \\
\hline $\mathrm{BD}+44493$ & -3.83 & 5.95 & 0.30 & 2.71 & 0.46 & 4.23 & Ito et al. (2013) \\
\hline BD-18 5550 & -3.2 & 6.05 & $\cdots$ & $\cdots$ & 0.49 & 4.89 & Roederer et al. (2014b) \\
\hline BS 16929-005 & -3.34 & 6.09 & 0.03 & 2.93 & 0.30 & 4.56 & Yong et al. (2013) \\
\hline CD-24 17504 & -3.41 & 6.12 & -0.23 & 2.60 & 0.34 & 4.53 & Jacobson et al. (2015) \\
\hline CS 22185-007 & -3.06 & 6.11 & -0.02 & 3.16 & 0.49 & 5.03 & Roederer et al. (2014b) \\
\hline CS 22189-009 & -3.92 & 5.51 & -0.10 & 2.22 & 0.44 & 4.12 & Roederer et al. (2014b) \\
\hline CS $22873-128$ & -3.32 & 5.90 & -0.11 & 2.81 & 0.66 & 4.94 & Roederer et al. (2014b) \\
\hline CS 22874-123 & -2.79 & 6.36 & 0.21 & 3.66 & 0.29 & 5.10 & Roederer et al. (2014b) \\
\hline CS 22877-001 & -3.31 & 6.67 & $\cdots$ & $\cdots$ & 0.38 & 4.67 & Roederer et al. (2014b) \\
\hline CS 22885-096 & -4.41 & 5.34 & 0.22 & 2.05 & 0.84 & 4.03 & Roederer et al. (2014b) \\
\hline CS 22888-031 & -3.71 & 5.64 & $\cdots$ & $\cdots$ & 0.48 & 4.37 & Roederer et al. (2014b) \\
\hline CS 22891-200 & -4.06 & 5.61 & $\cdots$ & $\cdots$ & 0.82 & 4.36 & Roederer et al. (2014b) \\
\hline CS 22892-025 & -2.78 & 6.41 & $\cdots$ & $\cdots$ & 0.35 & 5.17 & Roederer et al. (2014b) \\
\hline CS 22896-110 & -2.85 & 6.40 & $\cdots$ & $\cdots$ & 0.56 & 5.31 & Roederer et al. (2014b) \\
\hline CS 22897-008 & -3.83 & 5.92 & -0.05 & 2.36 & 0.60 & 4.37 & Roederer et al. (2014b) \\
\hline CS 22949-037 & -4.38 & 5.97 & $\cdots$ & $\cdots$ & 1.56 & 4.78 & Roederer et al. (2014b) \\
\hline CS 22949-048 & -3.55 & 5.81 & $\cdots$ & $\cdots$ & 0.40 & 4.45 & Roederer et al. (2014b) \\
\hline CS 22950-046 & -4.12 & 5.64 & $\cdots$ & $\cdots$ & 0.58 & 4.06 & Roederer et al. (2014b) \\
\hline CS 22953-037 & -3.21 & 6.22 & $\cdots$ & $\cdots$ & 0.55 & 4.94 & Roederer et al. (2014b) \\
\hline CS 22956-050 & -3.67 & 5.74 & $\ldots$ & $\ldots$ & 0.66 & 4.59 & Roederer et al. (2014b) \\
\hline CS 22957-013 & -2.98 & 6.17 & -0.08 & 3.18 & 0.48 & 5.10 & Roederer et al. (2014b) \\
\hline CS 22957-024 & -2.91 & 6.30 & -0.13 & 3.20 & 0.37 & 5.06 & Roederer et al. (2014b) \\
\hline CS 22958-083 & -3.05 & 6.09 & 0.07 & 3.26 & 0.59 & 5.14 & Roederer et al. (2014b) \\
\hline CS 22960-048 & -3.91 & 5.45 & $\cdots$ & $\cdots$ & 0.72 & 4.41 & Roederer et al. (2014b) \\
\hline CS 22964-183 & -3.01 & 6.25 & $\cdots$ & $\cdots$ & -0.07 & 4.52 & Roederer et al. (2014b) \\
\hline CS 22964-214 & -2.95 & 6.21 & $\cdots$ & $\cdots$ & 0.40 & 5.05 & Roederer et al. (2014b) \\
\hline CS 22965-054 & -3.17 & 6.28 & $\cdots$ & $\cdots$ & 0.25 & 4.68 & Roederer et al. (2014b) \\
\hline CS 29493-050 & -2.92 & 6.35 & -0.10 & 3.22 & 0.45 & 5.13 & Roederer et al. (2014b) \\
\hline CS 29502-092 & -3.3 & 6.59 & -0.02 & 2.92 & 0.51 & 4.81 & Roederer et al. (2014b) \\
\hline CS 29504-006 & -3.12 & 6.90 & $\cdots$ & $\cdots$ & 0.38 & 4.86 & Roederer et al. (2014b) \\
\hline CS 29514-007 & -2.83 & 6.49 & $\cdots$ & $\cdots$ & 0.22 & 4.99 & Roederer et al. (2014b) \\
\hline CS 30314-067 & -3.31 & 6.70 & $\cdots$ & $\cdots$ & 0.56 & 4.85 & Roederer et al. (2014b) \\
\hline CS $30492-110$ & -3.27 & 6.04 & $\cdots$ & $\cdots$ & 0.53 & 4.86 & Roederer et al. (2014b) \\
\hline HD 126587 & -3.29 & 5.98 & 0.06 & 3.01 & 0.61 & 4.92 & Roederer et al. (2014b) \\
\hline HD 237846 & -3.21 & 5.96 & $\cdots$ & $\cdots$ & 0.44 & 4.83 & Roederer et al. (2014b) \\
\hline HE $0015+0048$ & -3.07 & 6.65 & $\cdots$ & $\cdots$ & 0.66 & 5.19 & Hollek et al. (2011) \\
\hline HE $0020-1741$ & -4.05 & 6.12 & 0.59 & 2.78 & 1.03 & 4.58 & Placco et al. (2016) \\
\hline HE $0055-2314$ & -2.70 & 6.66 & -0.12 & 3.47 & 0.33 & 5.28 & Cohen et al. (2013) \\
\hline HE 0057-5959 & -4.08 & 5.21 & 1.98 & 4.14 & 0.51 & 4.03 & Yong et al. (2013) \\
\hline HE $0102-1213$ & -3.28 & 6.46 & -0.02 & 2.94 & 0.06 & 4.38 & Yong et al. (2013) \\
\hline HE 0103-0357 & -3.20 & 5.96 & 0.01 & 3.05 & 0.47 & 4.87 & Cohen et al. (2013) \\
\hline HE $0130-1749$ & -3.39 & 5.94 & 0.18 & 3.08 & 0.51 & 4.77 & Cohen et al. (2013) \\
\hline HE $0132-2429$ & -3.6 & 5.66 & -0.18 & 2.46 & 0.39 & 4.39 & Cohen et al. (2008) \\
\hline HE 0134-1519 & -3.98 & 5.45 & -0.24 & 2.02 & 0.25 & 3.87 & Hansen et al. (2014) \\
\hline HE $0139-2826$ & -3.46 & 5.83 & 0.46 & 3.24 & 0.56 & 4.70 & Placco et al. (2014a) \\
\hline HE 0146-1548 & -3.46 & 6.54 & 1.17 & 3.95 & 0.87 & 5.01 & Yong et al. (2013) \\
\hline HE $0302-3417 \mathrm{a}$ & -3.70 & 5.93 & $\ldots$ & $\ldots$ & 0.55 & 4.45 & Hollek et al. (2011) \\
\hline HE $0324+0152 a$ & -3.32 & 5.95 & $\cdots$ & $\cdots$ & 0.65 & 4.93 & Hollek et al. (2011) \\
\hline
\end{tabular}


YOON ET AL.

Table 2 (continued)

\begin{tabular}{|c|c|c|c|c|c|c|c|}
\hline Name & {$[\mathrm{Fe} / \mathrm{H}]$} & $A(\mathrm{C})$ & {$[\mathrm{Na} / \mathrm{Fe}]$} & $A(\mathrm{Na})$ & {$[\mathrm{Mg} / \mathrm{Fe}]$} & $A(\mathrm{Mg})$ & Reference \\
\hline HE 0401-3835 & -3.10 & 6.39 & 0.19 & 3.38 & 0.19 & 4.74 & Cohen et al. (2013) \\
\hline HE $0420+0123 a$ & -3.03 & 6.18 & $\cdots$ & $\cdots$ & 0.44 & 5.01 & Hollek et al. (2011) \\
\hline HE $0432-1005 a$ & -3.21 & 6.18 & $\cdots$ & $\cdots$ & 0.50 & 4.89 & Hollek et al. (2011) \\
\hline HE $0440-1049$ & -3.02 & 6.10 & -0.04 & 3.18 & 0.79 & 5.37 & Hansen et al. (2015a) \\
\hline HE $0557-4840$ & -4.73 & 5.30 & -0.25 & 1.26 & 0.16 & 3.03 & Norris et al. (2007) \\
\hline HE 1116-0634 & -3.73 & 5.51 & $\cdots$ & $\cdots$ & 0.82 & 4.69 & Hollek et al. (2011) \\
\hline HE $1124-2335$ & -3.36 & 6.07 & 0.02 & 2.90 & 0.59 & 4.83 & Roederer et al. (2014b) \\
\hline HE 1201-1512 & -3.92 & 6.11 & -0.35 & 1.97 & 0.20 & 3.88 & Yong et al. (2013) \\
\hline HE 1300-0641 & -3.14 & 6.55 & $\cdots$ & $\cdots$ & 0.02 & 4.48 & Barklem et al. (2005) \\
\hline HE $1300-2201$ & -2.60 & 6.80 & $\cdots$ & $\cdots$ & 0.27 & 5.27 & Barklem et al. (2005) \\
\hline HE 1311-0131 & -3.15 & 6.01 & $\cdots$ & $\ldots$ & 0.49 & 4.94 & Hollek et al. (2011) \\
\hline HE 1338-0052 & -3.06 & 6.90 & $\cdots$ & $\cdots$ & 0.46 & 5.05 & Cohen et al. (2013) \\
\hline HE 1351-1049 & -3.45 & 6.49 & $\cdots$ & $\cdots$ & 0.28 & 4.43 & Barklem et al. (2005) \\
\hline HE $1405-2512$ & -2.85 & 6.31 & -0.62 & 2.77 & 0.24 & 4.99 & Cohen et al. (2013) \\
\hline HE $1432-1819$ & -2.61 & 6.86 & -0.03 & 3.65 & 0.61 & 5.65 & Cohen et al. (2013) \\
\hline HE $1439-0218$ & -2.70 & 6.60 & -0.21 & 3.33 & 0.35 & 5.25 & Cohen et al. (2013) \\
\hline HE 1506-0113 & -3.54 & 6.38 & 1.65 & 4.35 & 0.89 & 4.95 & Yong et al. (2013) \\
\hline HE 2123-0329 & -3.22 & 6.27 & $\cdots$ & $\cdots$ & 0.58 & 4.96 & Hollek et al. (2011) \\
\hline HE 2138-0314 & -3.29 & 5.96 & $\cdots$ & $\cdots$ & 0.49 & 4.8 & Hollek et al. (2011) \\
\hline HE 2141-3741 & -3.30 & 5.96 & 0.29 & 3.23 & 0.48 & 4.78 & Placco et al. (2014a) \\
\hline HE $2142-5656$ & -2.87 & 6.61 & 0.81 & 4.18 & 0.33 & 5.06 & Yong et al. (2013) \\
\hline HE $2247-7400$ & -2.87 & 6.58 & 0.82 & 4.19 & 0.33 & 5.06 & Yong et al. (2013) \\
\hline HE 2302-2154a & -3.9 & 5.61 & $\cdots$ & $\cdots$ & 0.28 & 3.98 & Hollek et al. (2011) \\
\hline HE 2314-1554 & -3.33 & 5.89 & 0.0 & 2.91 & 0.67 & 4.94 & Cohen et al. (2013) \\
\hline HE 2318-1621 & -3.67 & 6.30 & 0.71 & 3.28 & 0.20 & 4.13 & Placco et al. (2014a) \\
\hline HE 2323-6549 & -3.35 & 5.81 & 0.59 & 3.48 & 0.55 & 4.80 & Placco et al. (2014a) \\
\hline HE 2331-7155 & -3.68 & 6.47 & 0.46 & 3.02 & 1.20 & 5.12 & Hansen et al. (2015a) \\
\hline LAMOST J1253+0753 & -4.02 & 6.00 & -0.20 & 2.02 & 0.24 & 3.82 & Li et al. (2015) \\
\hline SDSS J0140+2344 & -4.09 & 5.91 & 0.17 & 2.32 & 0.48 & 3.99 & Yong et al. (2013) \\
\hline SDSS J0741+6708 & -2.87 & 6.31 & 0.28 & 3.65 & 0.47 & 5.20 & Aoki et al. (2013) \\
\hline SMSS J0021-4711 & -3.17 & 6.04 & -0.13 & 2.94 & 0.43 & 4.86 & Jacobson et al. (2015) \\
\hline SMSS J0106-5244 & -3.79 & 5.49 & 0.29 & 2.74 & 0.57 & 4.38 & Jacobson et al. (2015) \\
\hline SMSS J0231-5239 & -2.94 & 6.26 & 0.87 & 4.17 & 0.68 & 5.34 & Jacobson et al. (2015) \\
\hline SMSS J0231-5753 & -3.42 & 6.08 & -0.17 & 2.65 & 0.29 & 4.47 & Jacobson et al. (2015) \\
\hline SMSS J0330-6813 & -3.44 & 6.07 & 0.73 & 3.53 & 0.63 & 4.79 & Jacobson et al. (2015) \\
\hline SMSS J0617-6007 & -2.72 & 6.45 & 0.41 & 3.93 & 0.4 & 5.28 & Jacobson et al. (2015) \\
\hline SMSS J0702-6004 & -2.62 & 6.68 & 0.61 & 4.23 & 0.45 & 5.43 & Jacobson et al. (2015) \\
\hline SMSS J1232-0545 & -3.03 & 6.18 & 0.45 & 3.66 & 0.56 & 5.13 & Jacobson et al. (2015) \\
\hline SMSS J1258-3350 & -3.44 & 5.70 & 0.53 & 3.33 & 0.53 & 4.69 & Jacobson et al. (2015) \\
\hline SMSS J1435-4203 & -3.15 & 6.26 & 0.42 & 3.51 & 0.49 & 4.94 & Jacobson et al. (2015) \\
\hline SMSS J1556-1655 & -2.79 & 6.44 & 0.39 & 3.84 & 0.52 & 5.33 & Jacobson et al. (2015) \\
\hline SMSS J1738-1457 & -3.58 & 6.18 & 0.62 & 3.28 & 0.43 & 4.45 & Jacobson et al. (2015) \\
\hline SMSS J1749-4551 & -2.77 & 6.50 & 0.51 & 3.98 & 0.47 & 5.30 & Jacobson et al. (2015) \\
\hline SMSS J1848-3059 & -3.65 & 5.75 & 0.40 & 2.99 & 0.49 & 4.44 & Jacobson et al. (2015) \\
\hline SMSS J1905-2149 & -3.11 & 6.03 & 0.46 & 3.59 & 0.45 & 4.94 & Jacobson et al. (2015) \\
\hline SMSS J2258-2654 & -3.49 & 6.03 & 0.06 & 2.81 & 0.06 & 4.17 & Jacobson et al. (2015) \\
\hline \multicolumn{8}{|c|}{ Group III CEMP-no Stars } \\
\hline G77-61 & -4.08 & 7.00 & 0.74 & 2.90 & 0.48 & 4.00 & Plez \& Cohen (2005) \\
\hline HE $0107-5240$ & -5.44 & 7.03 & 1.06 & 1.86 & 0.25 & 2.41 & Christlieb et al. (2004) \\
\hline HE 0233-0343 & -4.68 & 7.23 & $<0.50$ & $<2.06$ & 0.59 & 3.51 & Hansen et al. (2014) \\
\hline HE 1012-1540 & -4.17 & 6.67 & 1.65 & 3.72 & 1.81 & 5.24 & Roederer et al. (2014b) \\
\hline
\end{tabular}

Table 2 continued 
Table 2 (continued)

\begin{tabular}{lrrrrrrr}
\hline \multicolumn{1}{c}{ Name } & {$[\mathrm{Fe} / \mathrm{H}]$} & $A(\mathrm{C})$ & {$[\mathrm{Na} / \mathrm{Fe}]$} & $A(\mathrm{Na})$ & {$[\mathrm{Mg} / \mathrm{Fe}]$} & $A(\mathrm{Mg})$ & Reference \\
\hline HE 1310-0536 & -4.15 & 6.71 & 0.19 & 2.28 & 0.42 & 3.87 & Hansen et al. (2014) \\
HE 1327-2326 & -5.71 & 6.90 & 2.46 & 2.99 & 1.65 & 3.54 & Frebel et al. (2008) \\
HE 2139-5432 & -4.02 & 7.01 & 2.15 & 4.37 & 1.61 & 5.19 & Yong et al. (2013) \\
SDSS J1313-0019 & -5.00 & 6.41 & 0.37 & 1.61 & 0.44 & 3.04 & Frebel \& Norris (2015) \\
SDSS J1742+2531 & -4.78 & 7.26 & $<0.64^{\mathrm{a}}$ & $<2.10$ & $<0.21^{\mathrm{a}}$ & $<3.03$ & Bonifacio et al. (2015) \\
SMSS J0313-6708 & $<-7.8$ & 6.27 & $\ldots$ & $<0.56^{\mathrm{b}}$ & $>3.35^{\mathrm{c}}$ & $3.15^{\mathrm{b}}$ & Bessell et al. (2015) \\
\hline
\end{tabular}

Note- $A(\mathrm{C})$ is based on the corrected carbon abundance ratio, $[\mathrm{C} / \mathrm{Fe}]_{c}$; the listed references are the same as in Table 1. $A(\mathrm{Na})$ and $A(\mathrm{Mg})$ are obtained based on the Solar abundances of $\mathrm{Na}$ and $\mathrm{Mg}$ from Asplund et al. (2009).

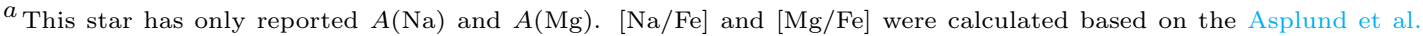
(2009) Solar abundance.

${ }^{b}$ This star has only reported $[\mathrm{Na} / \mathrm{H}]<-5.7$ and $[\mathrm{Mg} / \mathrm{H}]=-4.45$.

${ }^{c}[\mathrm{Mg} / \mathrm{Fe}]$ was calculated based on the reported $[\mathrm{Mg} / \mathrm{H}]-[\mathrm{Fe} / \mathrm{H}]$.

Table 3. Interesting Outliers among the CEMP Stars

\begin{tabular}{|c|c|c|c|c|c|c|c|c|}
\hline Name & {$[\mathrm{Fe} / \mathrm{H}]$} & $\begin{array}{c}A(C) \\
\text { (corrected) }\end{array}$ & {$[\mathrm{C} / \mathrm{Fe}]_{c}$} & {$[\mathrm{Ba} / \mathrm{Fe}]$} & {$[\mathrm{Eu} / \mathrm{Fe}]$} & $\begin{array}{c}\text { Class } \\
(\mathrm{Ba} \mid A(C))\end{array}$ & Binarity & Reference \\
\hline \multicolumn{9}{|c|}{ Single CEMP-no Stars with high $A(\mathrm{C})$, low $[\mathrm{Ba} / \mathrm{Fe}]$} \\
\hline CS 29498-043 & -3.87 & 7.62 & 3.06 & -0.49 & $<0.23$ & $\left(\begin{array}{ll}\text { no } & \mathrm{s}\end{array}\right)$ & 1 & Roederer et al. (2014b) \\
\hline HE $0405-0526$ & -2.18 & 7.17 & 0.92 & -0.22 & $\cdots$ & $($ no $\mid \mathrm{s})$ & 1 & Hansen et al. (2016b) \\
\hline HE 1133-0555 & -2.40 & 8.31 & 2.28 & -0.58 & $\cdots$ & $\left(\begin{array}{ll}\text { no } & \mathrm{s}\end{array}\right)$ & 1 & Hansen et al. (2016b) \\
\hline HE 1302-0954 & -2.25 & 7.37 & 1.19 & $<-0.53$ & $\cdots$ & $\left(\begin{array}{ll}\text { no } \mid & s\end{array}\right)$ & 1 & Hansen et al. (2016b) \\
\hline HE $1410+0213$ & -2.19 & 8.21 & 1.97 & -0.26 & $\cdots$ & $($ no $\mid \mathrm{s})$ & 1 & Cohen et al. (2013) \\
\hline \multicolumn{9}{|c|}{ Binary CEMP-no Stars with high $A(\mathrm{C})$, low $[\mathrm{Ba} / \mathrm{Fe}]$} \\
\hline CS 22957-027 & -3.19 & 7.87 & 2.63 & -0.81 & $<1.07$ & $\left(\begin{array}{ll}\text { no } \mid & s\end{array}\right)$ & 2 & Roederer et al. (2014b) \\
\hline HE 0219-1739 & -3.09 & 7.55 & 2.21 & $<-1.39$ & $\cdots$ & $\left(\begin{array}{ll}\text { no } \mid & s\end{array}\right)$ & 2 & Hansen et al. (2016b) \\
\hline HE $1150-0428$ & -3.47 & 7.35 & 2.39 & -0.48 & $<1.49$ & $\left(\begin{array}{ll}\text { no } & s\end{array}\right)$ & 2 & Yong et al. (2013) \\
\hline G $77-61$ & -4.08 & 7.00 & 2.65 & $<1.00$ & $>3.04$ & ( no $\mid$ no) & 2 & Plez \& Cohen (2005) \\
\hline \multicolumn{9}{|c|}{ Binary CEMP-no Stars with low $A(\mathrm{C})$, low $[\mathrm{Ba} / \mathrm{Fe}]$} \\
\hline CD-24 17504 & -3.41 & 6.12 & 1.10 & $<-1.05$ & $<1.16$ & ( no $\mid$ no) & 2 & Jacobson et al. (2015) \\
\hline HE 1506-0113 & -3.54 & 6.38 & 1.47 & -0.80 & $\cdots$ & ( no $\mid$ no) & 2 & Yong et al. (2013) \\
\hline \multicolumn{9}{|c|}{ Binary CEMP-s/rs Stars with low $A(\mathrm{C})$, high $[\mathrm{Ba} / \mathrm{Fe}]$} \\
\hline CS $22964-161 B^{a}$ & -2.39 & 7.44 & 1.40 & 1.30 & $\cdots$ & $(\mathrm{s} \mid \mathrm{s})$ & 2 & Thompson et al. (2008) \\
\hline HD 196944 & -2.41 & 7.33 & 1.31 & 1.23 & -0.11 & $(\mathrm{~s} \mid \mathrm{s})$ & 2 & Placco et al. (2015) \\
\hline HE 0430-1609 & -3.00 & 7.09 & 1.66 & 1.62 & $\cdots$ & ( $\mathrm{s} \mid \mathrm{no})$ & 2 & Hansen et al. (2016c) \\
\hline HE 0441-0652 & -2.77 & 7.29 & 1.63 & 1.20 & $\cdots$ & $(\mathrm{s} \mid \mathrm{s})$ & 2 & Yong et al. (2013) \\
\hline HE 1031-0020 & -2.79 & 7.29 & 1.65 & 1.61 & $<0.91$ & $(\mathrm{~s} \mid \mathrm{s})$ & 2 & Yong et al. (2013) \\
\hline HE 2312-0758 & -3.47 & 7.21 & 2.25 & 1.99 & $\cdots$ & $(\mathrm{s} \mid \mathrm{s})$ & 2 & Hansen et al. (2016c) \\
\hline \multicolumn{9}{|c|}{ Single CEMP-s/rs Stars with low $A(\mathrm{C})$, high $[\mathrm{Ba} / \mathrm{Fe}]$} \\
\hline CS 30301-015 & -2.73 & 7.44 & 1.74 & 1.70 & 0.22 & $(\mathrm{~s} \mid \mathrm{s})$ & 1 & Yong et al. (2013) \\
\hline HE $1045+0226$ & -2.25 & 7.38 & 1.20 & $\cdots$ & 0.27 & $(\mathrm{~s} \mid \mathrm{s})$ & 1 & Cohen et al. (2013) \\
\hline \multicolumn{9}{|c|}{ Single CEMP-s/rs Stars with high $A(\mathrm{C})$, high $[\mathrm{Ba} / \mathrm{Fe}]$} \\
\hline HE 0206-1916 & -2.52 & 8.03 & 2.12 & 1.61 & $\cdots$ & $(\mathrm{s} \mid \mathrm{s})$ & 1 & Yong et al. (2013) \\
\hline HE $1105+0027$ & -2.42 & 7.97 & 1.96 & $\cdots$ & 1.80 & $(\mathrm{~s} \mid \mathrm{s})$ & 1 & Barklem et al. (2005) \\
\hline HE 2330-0555 & -2.98 & 7.69 & 2.24 & 1.00 & $\cdots$ & $(\mathrm{s} \mid \mathrm{s})$ & 1 & Yong et al. (2013) \\
\hline
\end{tabular}


YOON ET AL.

Table 3 (continued)

\begin{tabular}{|c|c|c|c|c|c|c|c|c|}
\hline Name & {$[\mathrm{Fe} / \mathrm{H}]$} & $\begin{array}{c}A(C) \\
\text { (corrected) }\end{array}$ & {$[\mathrm{C} / \mathrm{Fe}]_{c}$} & {$[\mathrm{Ba} / \mathrm{Fe}]$} & {$[\mathrm{Eu} / \mathrm{Fe}]$} & $\begin{array}{c}\text { Class } \\
(\mathrm{Ba} \mid A(C))\end{array}$ & Binarity & Reference \\
\hline BD-01 2582 & -2.62 & 6.83 & 1.02 & 1.05 & 0.36 & ( $\mathrm{s} \mid \mathrm{no})$ & $\cdots$ & Roederer et al. (2014b) \\
\hline CS 22943-201 & -2.68 & 7.65 & 1.90 & -0.54 & $<0.73$ & $(\mathrm{no} \mid \mathrm{s})$ & $\cdots$ & Roederer et al. (2014b) \\
\hline CS 22958-042 & -3.40 & 7.59 & 2.56 & $<-0.61$ & $<1.54$ & $(\mathrm{no} \mid \mathrm{s})$ & $\cdots$ & Roederer et al. (2014b) \\
\hline CS 22960-053 & -3.64 & 6.43 & 1.64 & 1.03 & $<0.7$ & $(\mathrm{~s} \mid \mathrm{no})$ & $\cdots$ & Roederer et al. (2014b) \\
\hline HE $0007-1832$ & -2.84 & 8.46 & 2.87 & 0.09 & $<1.75$ & $($ no $\mid \mathrm{s})$ & $\cdots$ & Cohen et al. (2013) \\
\hline HE $0100-1622$ & -2.93 & 8.27 & 2.77 & $<-1.80$ & $<0.80$ & $(\mathrm{no} \mid \mathrm{s})$ & $\cdots$ & Hansen et al. (2015a) \\
\hline HE 0233-0343 & -4.68 & 7.23 & 3.48 & $<0.80$ & $<3.00$ & $(\mathrm{no} \mid \mathrm{s})$ & $\cdots$ & Hansen et al. (2016b) \\
\hline HE $1456+0230$ & -3.37 & 7.43 & 2.37 & -0.19 & $\cdots$ & $(\mathrm{no} \mid \mathrm{s})$ & $\cdots$ & Cohen et al. (2013) \\
\hline HE 2133-1426 & -3.37 & 7.06 & 2.00 & 2.34 & $\cdots$ & ( $\mathrm{s} \mid \mathrm{no})$ & $\cdots$ & Cohen et al. (2013) \\
\hline HE $2202-4831$ & -2.78 & 8.08 & 2.43 & -1.28 & $\cdots$ & $(\mathrm{no} \mid \mathrm{s})$ & $\cdots$ & Yong et al. (2013) \\
\hline HE 2208-1239 & -2.88 & 6.86 & 1.31 & 1.68 & 1.52 & $(\mathrm{r} / \mathrm{s} \mid \mathrm{no})$ & $\cdots$ & Hansen et al. (2015a) \\
\hline HE 2235-5058 & -2.70 & 6.8 & 1.0 & 2.4 & $\ldots$ & $(\mathrm{s} \mid \mathrm{no})$ & $\cdots$ & Hansen et al. (2016a) \\
\hline HE $2319-5228$ & -2.60 & 7.7 & 1.9 & $<-3.0$ & $\cdots$ & $(\mathrm{no} \mid \mathrm{s})$ & $\cdots$ & Hansen et al. (2016a) \\
\hline HKII 17435-00532 & -2.23 & 6.98 & 0.78 & 0.86 & 0.47 & $(\mathrm{r} / \mathrm{s} \mid \mathrm{no})$ & $\cdots$ & Roederer et al. (2008) \\
\hline SDSS J0212+0137 & -3.57 & 7.12 & 2.26 & 0.51 & $\cdots$ & $(\mathrm{no} \mid \mathrm{s})$ & $\cdots$ & Bonifacio et al. (2015) \\
\hline SDSS J1036+1212 & -3.20 & 6.70 & 1.47 & 1.17 & $\cdots$ & ( $\mathrm{s} \mid \mathrm{no})$ & $\cdots$ & Masseron et al. (2012) \\
\hline SDSS J1422+0031 & -3.03 & 7.11 & 1.71 & -1.18 & $\cdots$ & $(\mathrm{no} \mid \mathrm{s})$ & $\cdots$ & Aoki et al. (2013) \\
\hline SDSS J1613+5309 & -3.33 & 7.21 & 2.11 & 0.03 & $\cdots$ & $(\mathrm{no} \mid \mathrm{s})$ & $\cdots$ & Aoki et al. (2013) \\
\hline SDSS J1742+2531 & -4.78 & 7.26 & 3.61 & $<1.59$ & $\ldots$ & $(\mathrm{no} \mid \mathrm{s})$ & $\ldots$ & Bonifacio et al. (2015) \\
\hline
\end{tabular}

Note-The references for the claimed binarity are the same as in Table 1.

${ }^{a} \mathrm{CS} 22964-161 \mathrm{~A}$ has a higher reported $A(\mathrm{C})$ (7.64) than CS 22964-161B, by 0.2 dex, and is not listed in this table. 